Linköping Studies in Science and Technology. Thesis No. 1735

Licentiate Thesis

\title{
Simulation Based Evaluation of Advanced Driver Assistance Systems
}

Roya Elyasi-Pour

Department of Science and Technology

Linköping University, SE-601 74 Norrköping, Sweden

Norrköping 2015 
Simulation Based Evaluation of Advanced Driver Assistance Systems

Roya Elyasi-Pour

LIU-TEK-LIC 0280-7971

ISBN: 978-91-7685-887-5

ISSN 0280-7971

Linköping University

Department of Science and Technology

SE-601 74 Norrköping

Printed by LiU Tryck, Linköping, Sweden 2015 


\section{Abstract}

Road transportation is an essential element of mobility in most countries and we can observe an increasing demand for both goods and passenger traffic. There are however important societal and economical problems related to road transportation in terms of congestions, traffic safety and environmental effects. During the last decades vehicles have increasingly been equipped with different types of Advanced Driver Assistance Systems (ADAS). These systems can to some extent compensate for human behaviour and errors that cause congestions, accidents and air pollution. Most studies conducted to evaluate ADAS have focused on ADAS impacts on the driver or on the vehicle. Since an ADAS might influence not only driving behaviour and vehicle dynamics, but also the interaction between equipped and non-equipped vehicles, it is also important to consider the resulting effect on the traffic system. A reliable and realistic evaluation approach needs to include estimations of drivers' decisions in different traffic situations with respect to the ADAS functionality and how such decisions affect the traffic system as a whole.

The overall aim of the thesis is to develop a simulation based evaluation framework for investigations of impacts of different types of cruise controllers on the traffic system. The objective is also to apply the framework to evaluate a fuel minimizing cruise controller for trucks, the Look Ahead Cruise Control (LACC). The framework developed consists of a combination of a microscopic traffic simulation model, and a vehicle and ADAS simulation model. When applied for a specific ADAS, as for example the LACC, the framework needs to be complemented with a driver model that captures the changes in driving behaviour due to the system of interest. In this thesis a driver model for LACC equipped trucks was developed based on results from a driving simulator experiment, a field operational test, and a focus group study. Simulation experiments were carried out to observe the LACC impacts on the traffic system with respect to penetration rate, traffic density, and variation in the desired speed. Environmental effects were estimated using emission calculations.

The results show that an increase in traffic flow influences LACC-trucks more than CC-trucks with respect to fuel consumption and emissions. However, the results also show lower fuel consumption and emissions for LACC-trucks despite increased traffic flow. Increased penetration rate of LACC-trucks does not show any negative effect on traffic efficiency. 


\section{Acknowledgments}

This research was carried out at the division of Communication and Transport Systems (KTS) at Linköping University and the Swedish National Road and Transport Research Institute (VTI). Vinnova/FFI - Transport efficiency is greatly appreciated for financing this research.

First of all, I would like to express my gratitude to my supervisors Prof. Jan Lundgren and Johan Olstam for their guidance and support. I am thankful to all the colleagues at VTI and KTS for a stimulating working environment. A special thanks to Prof. Maud Göthe-Lundgren for all support and encouragement.

I would also like to thank Linus Bredberg, Johan Brodin and Mikael Ögren (Scania) for invaluable development work and assistance in the interconnection of the vehicle simulation and the traffic simulation models.

Finally I would like to thank all my family and friends for always supporting me. Dena and Bahar, my beautiful daughters! Thank you for being a wonderful source of love and energy. You mean the world to me!

Linköping, October 2015

Roya Elyasi-Pour 


\section{CONTENTS}

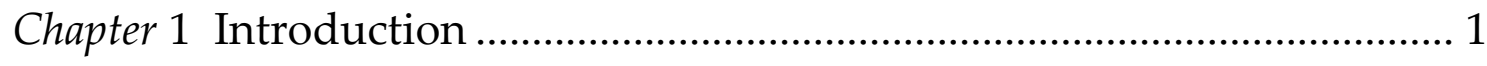

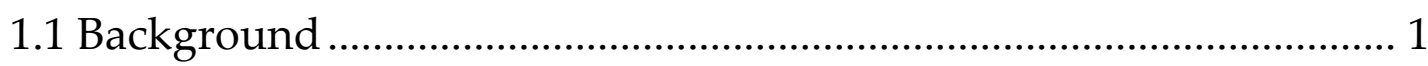

1.2 Aim

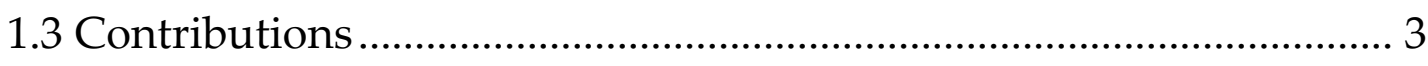

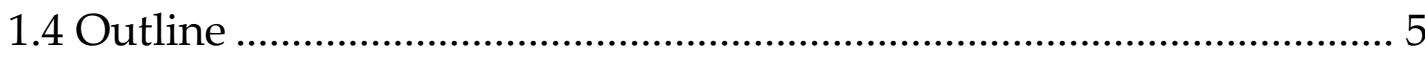

Chapter 2 Advanced Driver Assistance Systems (ADAS) ……………......... 7

2.1 Intelligent Transport Systems ................................................................ 8

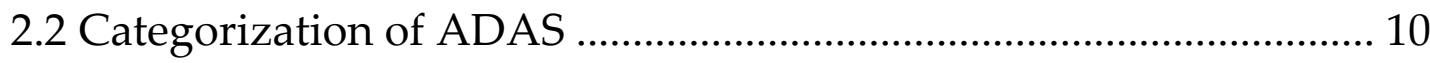

2.2.1 Decision process during driving ................................................... 11

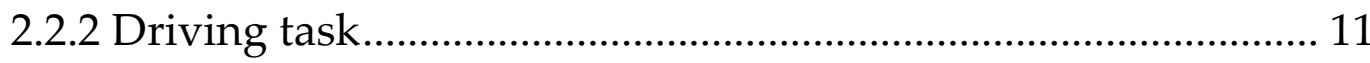

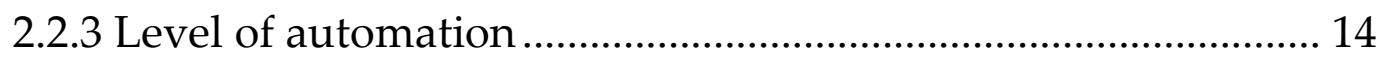

2.2.4 Autonomous or Cooperative..................................................... 15

2.3 Cruise Controllers ................................................................................. 15

2.4 The Look Ahead Cruise Control......................................................... 18

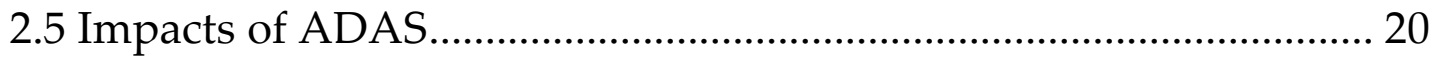

2.5.1 ADAS vehicle impacts............................................................... 21

2.5.2 ADAS impacts on the driver ....................................................... 24 
2.5.3 ADAS impacts on the traffic system ........................................... 26

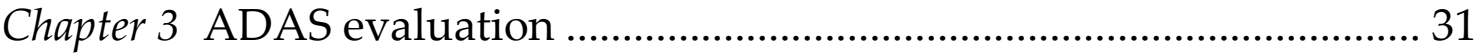

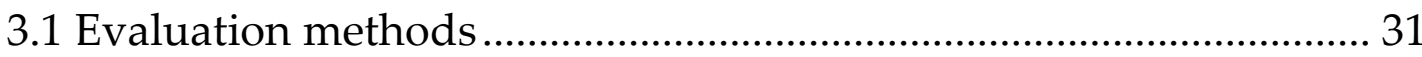

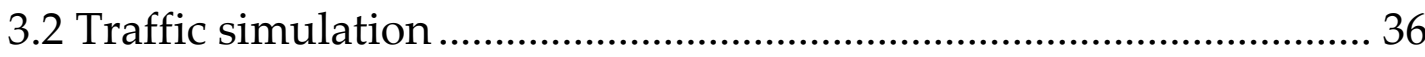

3.2.1 Car-following models .................................................................. 37

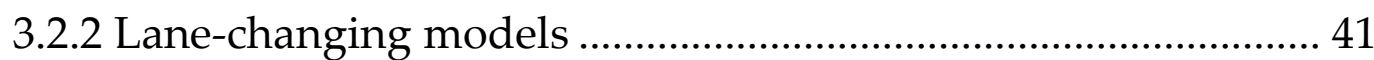

3.3 Traffic simulation of ADAS ........................................................... 42

Chapter 4 Simulation based evaluation framework ..................................... 49

4.1 Combining simulation methods ........................................................... 50

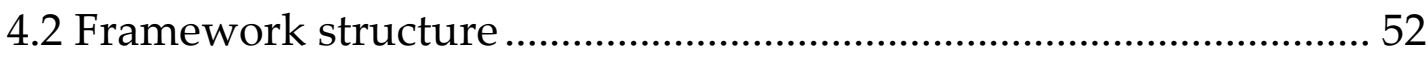

4.2.1 The traffic simulation ................................................................. 52

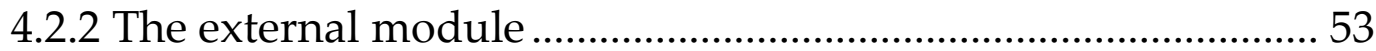

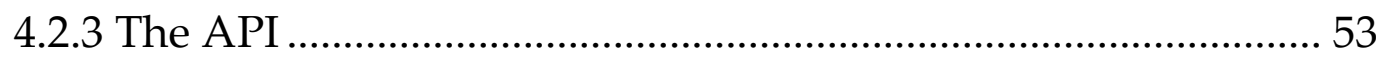

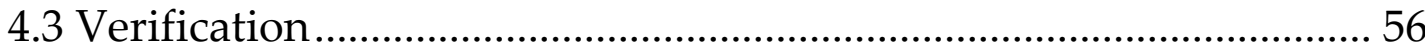

4.3.1 Single-vehicle trajectories ....................................................... 57

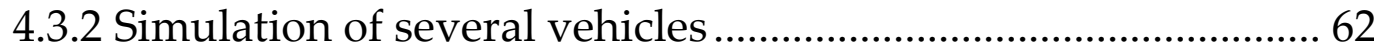

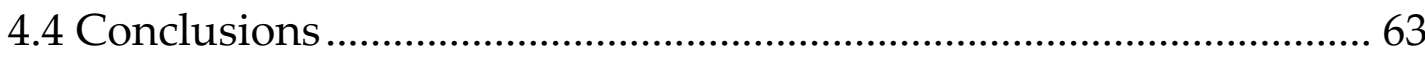

Chapter 5 Driving behaviour modelling........................................................ 65

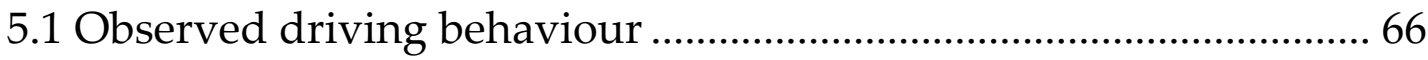

5.1.1. Driving Simulator Study and Field Observation Test.............. 66

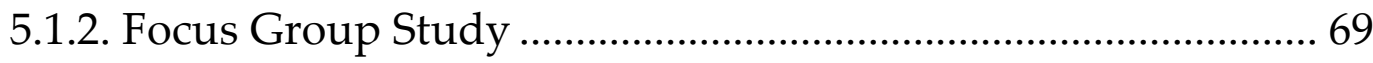

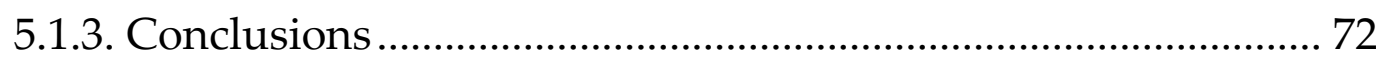

5.2 Driver model for de/reactivation of LACC ……………………........ 72

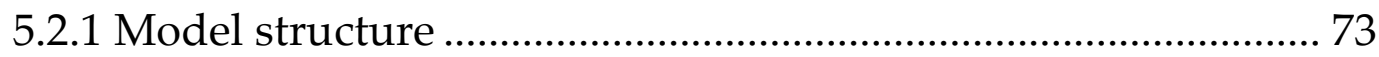

5.2.1. The following situation .............................................................. 75

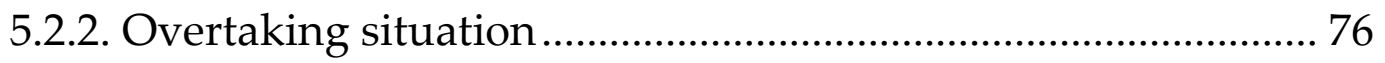

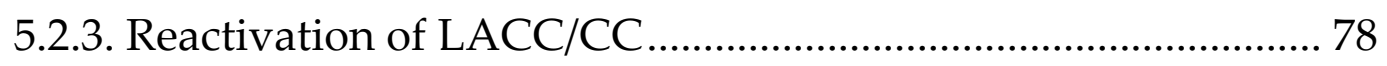

5.3 Verification of the driver model ....................................................... 78 
5.3.1. Verification of collision free simulations .................................... 79

5.3.2. Verification of deactivation and reactivation............................. 79

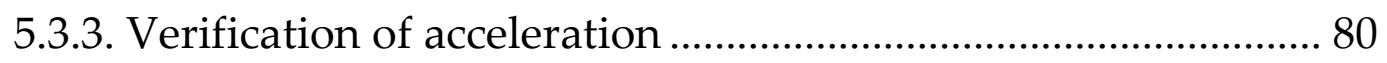

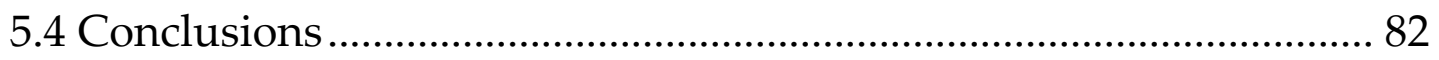

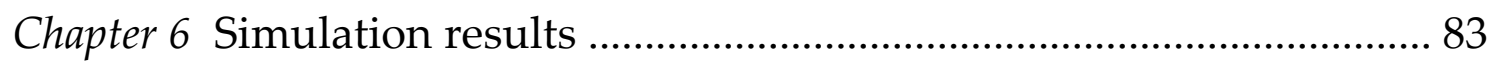

6.1 Design of traffic simulation experiments .......................................... 83

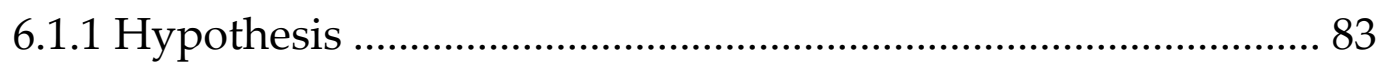

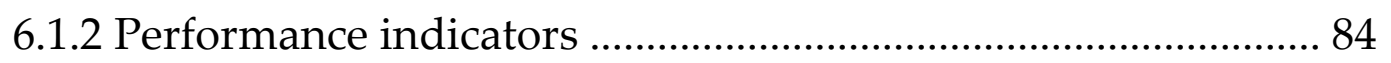

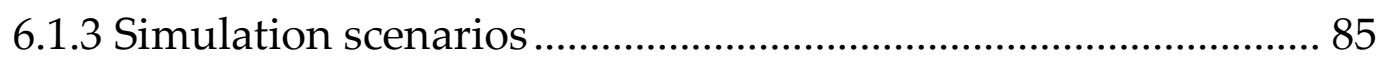

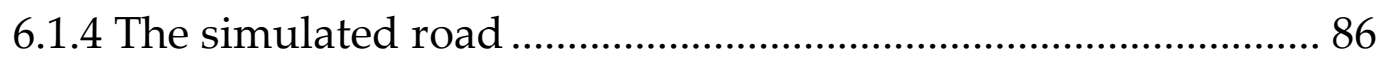

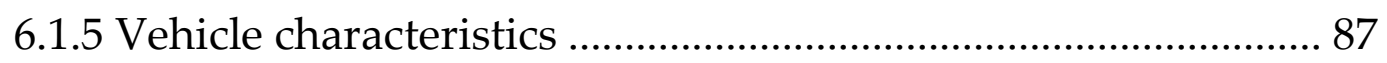

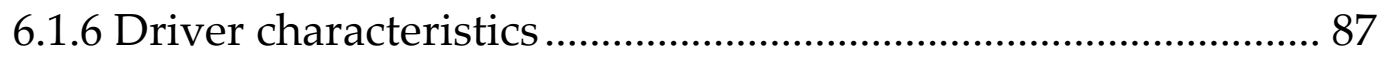

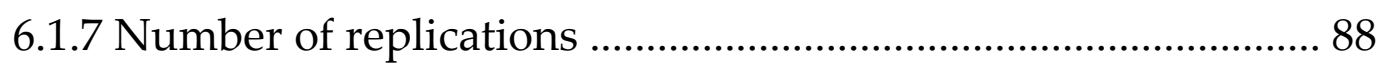

6.2 Impacts on the environment................................................................ 88

6.2.1 Impacts on fuel consumption ..................................................... 89

6.2.2 Impacts on emissions.................................................................. 98

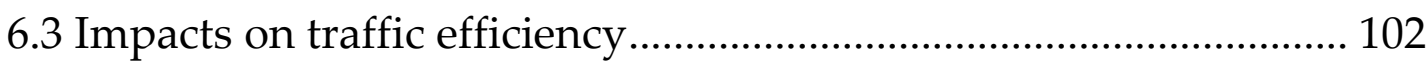

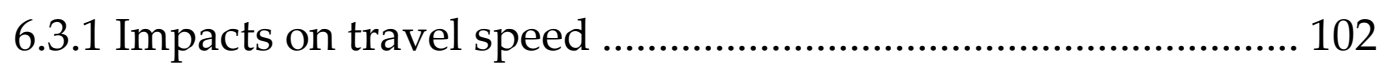

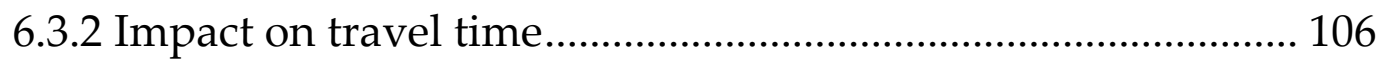

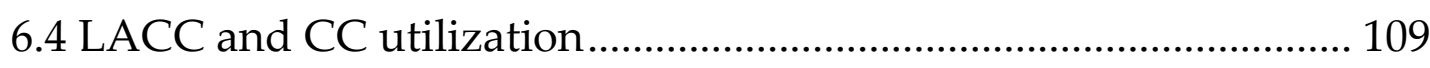

6.5 Discussion and conclusions ............................................................... 111

Chapter 7 Discussion and future research................................................ 113

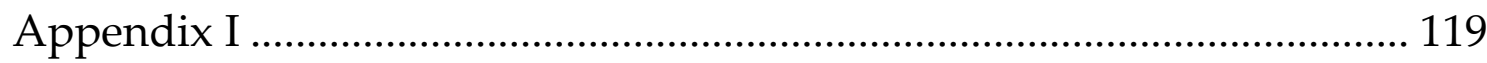

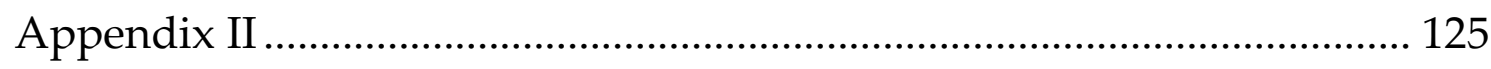

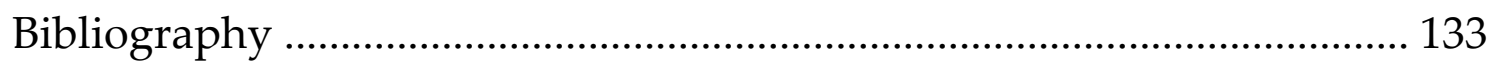




\section{Chapter 1}

\section{Introduction}

\subsection{Background}

As a consequence of globalization and economic development around the world, the demand for road transportation has increased. Road transportation is an essential element of mobility in most countries and constitutes a significant part of transportation of both passengers and goods. However, there are important societal and economical problems related to road transportation in terms of congestions, traffic safety and environmental effects. New technologies have been developed during the last decades to improve vehicles and infrastructure in order to achieve more efficient, safe and environmental friendly traffic systems. In this context, the development of Intelligent Transport Systems (ITS) has been a solution to improve traffic systems.

One important type of ITS is Advanced Driver Assistance Systems (ADAS), aimed at supporting the driver, improving road safety, driving performance and decreasing environmental effects. Vehicles have increasingly been equipped with different types of ADAS. As a result, the development of ADAS 
has become an important element in the traffic system. Advanced technology for information collection, data processing, decision support and automation has been utilized in the development of various ADAS. As ADAS have become a common factor in traffic, it is important to have knowledge about the corresponding effects and find out the actual contribution of these systems. An improved transport system is expected by using ADAS, since the increased quantity and quality of the information during driving, supports the drivers' driving tasks. However, this is dependent on the design of the ADAS and how non-equipped vehicles are affected.

It is expected that ADAS can compensate for human behaviour and errors that cause accidents, congestions, and air pollution, and many evaluation studies have been conducted to verify if ADAS meet the expectations. Most studies have generally been focused on ADAS impacts on the driver or on the vehicle. Examples include how changes in driving style influence fuel consumption or traffic safety, and how ADAS affects vehicle dynamics such as speed, acceleration, deceleration, etc. Furthermore, emission requirements have become stricter and the transportation sector endeavours to make transportation more resource efficient. In addition, truck costs are a significant part of the transport costs for the transport companies and truck owners. According to Hellström (2010) fuel costs for a class 8 truck, weighting more than 15 tones, are about $1 / 3$ of the life cycle cost. Load characteristics, travel distance, truck configurations, road geographical characteristics and driving practices influence the truck costs.

An important question in this thesis is how an ADAS effects the traffic system at an aggregated level. More specifically, the environmental effects are of interest. We have studied fuel minimizing ADAS for trucks that are designed to reduce the energy consumption for the equipped vehicle. The benefits of a fuel minimizing ADAS are more significant in heavy vehicle driving, due to the truck's large mass compared to the engine power. However, it is still important to observe the impacts on other elements in the traffic systems, e.g. the driver and the surrounding vehicles (both trucks and other types of vehicles). This allows us to estimate the real effect of the ADAS on the total energy consumption and emissions in the traffic system. 
An increased number of vehicles including ADAS-vehicles on the roads, and the complexity of ADAS systems have induced many questions about the corresponding effects on the traffic system.

\subsection{Aim}

The main motivation for this thesis is the need for an accurate evaluation model and tool for evaluation of ADAS. The overall aim of the thesis is to develop a simulation-based evaluation framework in order to investigate different types of fuel controllers' impacts, focusing on fuel minimizing cruise controllers for trucks. The objective is also to apply the framework to evaluate a fuel minimizing cruise controller for trucks, the Look Ahead Cruise Control (LACC).

In order to achieve an enhanced evaluation of ADAS, the framework is developed by combining microscopic traffic simulation modelling, vehicle and ADAS simulation modelling and emission calculations. The effects of ADAS on the traffic systems cannot be discussed without including considerations about how ADAS influence the driver and the vehicle. The developed framework allows us to consider different traffic conditions while taking into account the functionality of ADAS and variations in driving behaviour. A traffic simulation model is used as the core in the framework, due to its capability to commit various traffic situations and interaction between the vehicles. Vehicle simulation is integrated with traffic simulation in order to update the vehicle conditions influenced by ADAS. Emissions are calculated using a fuel and emission model based on speed profiles from the traffic simulation.

\subsection{Contributions}

The specific contributions of the thesis are as follows:

- An investigation of approaches and methods for evaluation of ADAS impacts.

- Development of a simulation based framework for estimations of ADAS impacts on the traffic system, by considering interaction between the driver, the vehicle and the traffic system. 
- Development of a driver model that takes into account the drivers' interaction with a fuel minimizing cruise controller for trucks.

- Implementation and verification of the developed framework and driver model.

- Application of the framework for a fuel minimizing cruise controller for trucks.

- Investigation of the environmental and traffic efficiency effects of a fuel minimizing cruise controller, using the developed framework.

Parts of the content have been presented in the following publication:

Olstam, J. and Elyasi-Pour, R. (2013). "Combining traffic and vehicle simulation for enhanced evaluations of Advanced Driver Assistance Systems". In proceedings of $16^{\text {th }}$ International IEEE Conference on Intelligent Transportation Systems (ITSC 2013).

Parts of the contents and the results have also been presented by the author at the following conferences:

- "An evaluation of an Environmental Driver Support System using interacted traffic and vehicle simulation", Nationella konferensen i transportforskning, Gothenburg, October, 2013

- "Driving behavior model in a simulation based evaluation approach for Advanced Driver Assistance Systems", Transportforum, Linköping, Sweden, January, 2014

- "Driving behavior model in a simulation based evaluation approach for Look Ahead Cruise Control", Nationella konferensen i transportforskning, Norrköping, October, 2014

- "Driving behavior model in a simulation based evaluation approach for look ahead cruise control", Transportforum, Linköping, Sweden, January, 2015 


\subsection{Outline}

An overview of different types of ADAS including a detailed description of the ADAS that will be used as a test case in the thesis, are presented in Chapter 2. Chapter 3 describes evaluation methods for ADAS and the need of a more holistic evaluation framework. The simulation based framework, both the structure and sub-models, is described in Chapter 4. The procedure of developing the driver model and the model itself are presented in Chapter 5. Chapter 6 contains the design of the simulation experiments, the computational results and analyses. Finally, Chapter 7 discusses the most important results of this thesis and the direction for further research. To provide more clarification, the thesis is followed by two appendices. Appendix I presents the set-up for the Focus Group Study and summarizes the findings. Appendix II contains graphs and tables that show detailed simulation outputs of the emissions, fuel consumption, and travel time. 
Chapter 1. Introduction 


\section{Chapter 2}

\section{Advanced Driver}

\section{Assistance Systems}

\section{(ADAS)}

Advanced Driver Assistance Systems (ADAS) are in-vehicle applications developed to assist the driver in controlling a vehicle. ADAS may control braking, maintain a proper speed, inform the driver about other vehicles or keep the vehicle in the correct lane. General aims of ADAS are increased traffic safety, better driving performance and reduced environmental effects of traffic. ADAS is one category of Intelligent Transport Systems (ITS) that is an essential part of today's traffic system. In this chapter we describe which impacts ADAS can have on the vehicle, the driver, and the traffic system. We start by giving a short introduction to ITS. 


\subsection{Intelligent Transport Systems}

Intelligent Transport Systems (ITS) is a term for a variety of new technologies, applications and operation methods for controlling and improving vehicles and the traffic system. These technologies provide operators with information about the transport system that can be used for better planning and more efficient operations. The purpose of ITS is to collect, establish, and distribute information about traffic and vehicle conditions in order to support the operation of transport networks as well as the control of vehicles. Various categorizations of ITS have been suggested by researchers. Sivaraj et al. (2012) presents the following categories:

- Advanced Traffic Management Systems (ATMS)

- Advanced Traveller Information Systems (ATIS)

- Advanced Public Transportation Systems (APTS)

- Commercial Vehicles Operation (CVO)

- Advanced Vehicle Control Systems (AVCS)

Advanced Traffic Management Systems (ATMS) are designed to improve traffic efficiency by providing road users with real-time traffic information. The system collects roadway and traffic information by using a combination of onboard tools, global positioning system (GPS) and dynamic central database of traffic problems, congestion and delays. In-vehicle displays, the car radio or portable communication devices inform drivers about the incidents and congestions ahead. Real-time traffic monitoring, dynamic signs, sign monitoring and control, traffic camera and ramp metering are examples of ATMS applications. Bertini et al. (2004) state that evaluations of ATMS such as ramp metering systems have confirmed improvements in safety, reduction in travel time and delay, and improved traffic throughput. Incident management systems are another example of ATMS that are designed to reduce the duration of incidents, such as crashes, breakdowns or other events that occur on the highways or freeways. Generally, incident management systems are based on tools for incident detection, verification and response to the incident. 
Advanced Traveller Information Systems (ATIS), are aimed at informing travellers about traffic conditions and influencing travellers' behaviour in a way that improves traffic safety and efficiency. Information is obtained from different data sources such as traffic reports, scheduled traffic events, sensors and maps, and are used by ATIS in order to assist the travellers in planning and decision making. Recently, developments in telecommunication technology have promoted evolution in ATIS deployment. Real-time traffic information about weather and road conditions, road constructions, etc., help the road users to plan departure time, route and mode choice. According to Chorus et al. (2006) an expectation is that ATIS affect the travellers' choice of destinations and activity patterns. From an individual traveller's perspective, the information presented by ATIS should generate alternative choices that provide maximum efficiency in time and costs. The travel information that is obtained before a travel choice is made, may stimulate changes in travellers' choice of mode, travel route and departure time. This is therefore of interest to policy-makers, public transport companies and the automotive industry.

Advanced Public Transportation Systems (APTS) apply transportation management and information technologies in order to increase the efficiency of operation and improve the safety of public transportation. The benefit of improved communication about disruptions in the traffic system is reduced cost and higher traveller satisfaction. In public transport, scheduling and routing decisions cause disturbances for the travellers and other operators. The traveller can receive updated information such as the current traffic status and any changes in the timetable or any incidents on public transport and indication to delays. Various applications have been developed to support the passenger in being aware about public transportation in cities. According to Chaves et al. (2011), results from several investigations show that better accessibility to information renders the system more attractive. Different devices, such as information centres, display at bus stations, smartphones and interactive TV have been used for this reason.

Commercial Vehicles Operation ( $\mathrm{CVO}$ ) are dynamic fleet management tools that can improve the efficiency of fleet operations by predicting supplies of vehicles and demands for services, based on current conditions. CVO include technologies that support the management of commercial vehicles with respect 
to speed, cost, stopping time and destination. Activities related to roadside operations, safety assurance, fleet and freight management are monitored in $\mathrm{CVO}$ to support commercial vehicle operations. Technologies such as electronic logbooks, automatic vehicle automation (AVL), automatic vehicle identification (AVI) and navigation devices are used in CVO.

Advanced Vehicle Control Systems (AVCS) are developed to improve road capacity and safety by reducing human driver error. AVCS enable drivers to detect and avoid risks and congestions with warning systems, automatic steering systems, trip routing and scheduling, control merging of traffic or collision avoidance. AVCS integrate sensors, computers and control systems to deal with the information chain between the transportation system and the driver in order to replace some of the human driver decisions and actions.

Advanced Driver Assistance Systems (ADAS) is a sub-class of AVCS, aimed at supporting drivers by either providing warnings to reduce risks and errors, or automating some of the driving tasks. Advanced cruise controllers, automated steering control for lane keeping, automated stopping and lane changes are examples of ADAS.

There has been an evident growth worldwide in the development of ADAS thanks to improvements in information technologies, computing and sensing. The development of ADAS is driven mainly by the car manufacturers since ADAS is considered as an important competitive improvement in vehicles. Furthermore, public authorities, road operators and societies have perceived that ADAS provide possibilities for smoother traffic flow, less congestions and more environmentally friendly traffic.

\subsection{Categorization of ADAS}

The range of research, development and evaluation of ADAS is broad and a variety of categorizations possible. I this section we give an overview of different suggested categorizations, based on the following aspects:

- Decision process during driving

- Driving tasks

- Level of automation 
- Autonomous or Cooperative systems

\subsubsection{Decision process during driving}

According to Michon (1985), driving is conducted in a social and technical environment and traffic should be treated as a system that includes many components such as road users, vehicle control systems, infrastructure and road control systems. This means that, executing driving tasks requires interaction between the driver and other components in the traffic system. In this context, it is possible to distinguish three levels of decision making in the driving process: strategic, tactical and operational level, where the levels correspond to different types of driving decisions. A hierarchal model is presented by Michon (1985):

- The strategic level: This level involves the general planning stage of a trip in terms of travel pattern (origin and destination), departure time, choice of modality and route planning.

- The tactical level: The dynamic planning of a trip such as minimizing the travel time, planning the optimal route, avoiding congestions or reducing the environmental effect, and sensing the tactical level. This level deals with interactions with other road users and traffic environment, such as overtaking or distance keeping.

- The operational level: This level concerns vehicle handling during a trip, such control as steering, acceleration and braking.

\subsubsection{Driving task}

According to Minderhoud et al. (1999) the hierarchal framework presented in the previous section is insufficient for identifying sub-tasks supported by ADAS. The sub-tasks that are dealing with vehicle control in relation with the driver, the road and other road users, requires another driving task model. In order to specify the control mechanisms that can be taken over by or influenced by ADAS, another framework is therefore presented by Minderhoud et al. (1999). The proposed model consists of: the route navigation sub-task, the lateral and longitudinal roadway sub-task and the lateral and longitudinal vehicle 
interaction sub-task. In addition, the roadway conditions and interaction with other vehicles are considered. Note that the focus in this model is on ADAS that are related to tactical and operational levels of the driving tasks.

Another general categorisation of ADAS, based on driving tasks is presented in AIDE (2005) and includes:

- Lateral control systems

- Longitudinal control systems

- Reversing aids / parking aids

- Vision enhancement systems

- Driver monitoring systems

- Pre-crash systems

- Road surface / low friction warning systems

Lateral Control systems improve road safety by avoidance of risky lane departure. The system will warn the driver when the vehicle is deviating from the lane and will provide braking or steering to keep the vehicle in correct lateral position. Almost all lateral control systems use magnetic nails or tape on the road and magnetic sensors in the vehicle or a frontal camera to identify the vehicles lateral position on the road. Different warnings systems are implemented such as wheel vibration or acoustic warning signals. One example of Lateral control systems is the Lane Keeping Assistant which detects the boundary of the lane and applies corrective wheel steering. Another example is the Lane Change Assistant that has warning system characteristics and includes the blind spot monitoring to detect the vehicle in the driver's blind spot. It will warn the driver if a lane-change is not safe due to too short distance between the vehicles in the target lane. An active overtaking assistant which considers the whole overtaking procedure, includes both monitoring and steering tasks, and affects both the driver and the vehicle.

Longitudinal Control systems support the driver in keeping the longitudinal speed limit, minimum safety distance to the vehicle ahead, or minimizing the fuel consumption. The aim is to control the speed and it can be based on input by the driver (i.e. the driver sets the desired speed) or the traffic system (i.e. traffic signs). In order to control the speed, a sensor based technology can be 
utilized in longitudinal control systems aimed at measuring preceding vehicles speed or estimating the distance to preceding vehicles. Forward Collision Warning system (FCW) and Cruise Controllers (CC) are examples of ADAS with longitudinal control purpose. The aim of FCW is to alert drivers in avoiding or reducing the severity of crashes. This means that the system does not have the power to control the vehicle. It only attempts to warn the driver to avoid rear-end collision. FCW monitors the forward area and provides a warning to the driver in the form of sound signals, visual signals, seat vibration or slight seatbelt tensioning. CC are further described in Section 2.3.

Reversing aids / parking aids are sensor based features, aimed at detecting obstacles while driving at very low speed. A visual display or acoustical system warns and informs the driver about the parking scenario. The system helps the driver to see or perceive the areas around the vehicle and warns the driver if there is risk of collision.

Vision enhancement systems are human-machine interfaces that improve the driver's vision. Sensors such as ultrasonic or video image processing, cameras, and radars are commonly used in these systems. Vision enhancement systems prepare high definition video displays, often in colour, based on the pictures that have been captured by cameras. For example, a night vision system provides an enhanced vision by monitoring a dimly lighted scene with true colours. This technology is especially beneficial in highway driving, during a reversing manoeuvre, night-time or foggy weather driving. However it is up to driver to take advantage of the information and display images while driving.

Driver monitoring systems purpose is to monitor the driver's physiological status and to warn and if needed alert the driver. States such as eye movements, heart rate variability, and lack of attention can for example be monitored.

Pre-crash systems (PSS) are designed to identify and detect when an accident is unavoidable and attempt to increase the safety on-board. PSS evaluates a vehicle's position as well as other objects on the road, in order to prevent damage that may be caused by an accident. A collision prediction unit estimates the collision between the equipped vehicle and another vehicle that has entered in the monitored area. Unlike other ADAS whose general aim is to avoid collisions, a pre-crash system will be active when an accident is prevailing. 
Accelerometers, radar sensors and data processing concepts are used to monitor potential collision situations. Pre-crash systems have basically two general aspects: distribution of collision energy in restraints and activating airbags, and seatbelt tension to increase safety.

Road surface / low friction warning systems transmit the road surface status to a warning system in the vehicle. These systems are designed for example to keep the wheels from locking up, distribute the braking pressure and load, or maintain requisite speed during downhill driving.

\subsubsection{Level of automation}

Level of automation is another basis for categorization of ADAS. Broqua (1991) has presented a categorization divided into Informing, Warning, Overrulable and Non-overrulable assistance systems. An increasing complexity can be noted in this categorization.

- Informing ADAS informs the driver about the vehicles position, speed etc. by using a display or speaker.

- Warning ADAS is thought to support the driver in risky situations. However, the driver has to make the appropriate decision when using the informing and warning ADAS.

- Overrulable ADAS takes over some driving tasks automatically but the driver can take back vehicle control while the system is activated.

- Non-overrulable ADAS is designed to replace a specific driving task and if the system identifies an emergency situation, it will decide if the driver should take over the driving task or a part of it. Differences between overrulable and non-overrulable systems can be imprecise, since an emergency stop functionality is available in non-overrulable systems and the driver is able to switch off the system, if needed. A non-overrulable ADAS' purpose is to manage a driving task completely, but in some situations the system decides that the driver should retake control. 


\subsubsection{Autonomous or Cooperative}

Piao et al. (2008) have compared ADAS in terms of technologies used in the systems from an operational point of view, and divided ADAS in autonomous and cooperative approaches. Most of ADAS mentioned in this chapter are autonomous ADAS which are in-vehicle control systems. The autonomous systems are designed to use the information about the equipped vehicle. Thanks to improvements in communication technologies during the last decades, cooperative systems have been developed as an extension of Intelligent Transport Systems (ITS). The idea is to use the communication technologies for give-and-take information between the vehicles and the infrastructure. The aim is to achieve a safer and smoother traffic environment by using the cooperative systems. With a cooperative ADAS, individual vehicles can relate to other vehicles and the environment. Cooperative systems are based on vehicle-tovehicle, vehicle-to-infrastructure or infrastructure-to-infrastructure communication. According to Van Arem et al. (2006), one of the potential advantages of vehicle-to-vehicle communication is to support longitudinal control from a leading vehicle. Moreover, communication between vehicles has improved road safety and reduced drivers' response time.

\subsection{Cruise Controllers}

Cruise controllers are overrulable driver assistance systems for longitudinal control of vehicles and are designed to maintain a reference speed automatically. According to Minderhoud (1999), the purpose of this kind of system is "increasing the comfort level of driver and improving the performance of the longitudinal driving task by assisting the longitudinal vehicle interaction driving task". The driver sets the reference speed and the cruise controller takes over control of the throttle and engine power. In highway and motorway driving, cruise controllers are the most commonly used driver support system. Apart from improved driving comfort, using cruise control can in some cases be the most fuel efficient way of driving.

The very first models of cruise controllers, which also were very simple, were used in cars as early as 1900 and the modern cruise controller was invented by Teetor (1950). This system calculated the throttle position that was needed to 
keep a constant speed. The driver had to increase the speed manually and activate the cruise controller.

Recently, further developments of cruise controllers have been introduced on the market, aimed at accomplishing both car-following and speed-regulation tasks. Cruise controllers contain an electronic throttle control that is widely applied in the engine control system and is known as one of the crucial technologies of the engine control system that regulates vehicle performance and emission levels. Therefore many researchers have tried to find techniques to improve these systems. This section introduces examples of cruise controllers that have been developed during the last decades.

\section{Regular Cruise Control}

The reference speed in these systems is a constant value based on a preferred speed value set by the driver. The speed will be controlled towards the reference speed and acceleration or deceleration is selected based on the current load and engine speed. In varied and congested traffic conditions, it is not possible to have the CC activated the entire time, due to too many interactions with constraining vehicles. In this thesis the term CC refers to regular cruise control.

\section{Adaptive Cruise Control}

Adaptive Cruise Control (ACC) is an extension of CC aimed at controlling the speed if the preceding vehicle is slower. ACC provides the possibility to control speed even in dense traffic and contains radars and sensors that regulate speed depending on vehicle condition as well as the distance to the vehicle in front. The aim of the ACC is a partial automation of longitudinal vehicle control that reduces the workload of the driver in dense traffic. ACC as other CC's is an overrulable ADAS and always works in cooperation with the driver. Delay due to the driver's reaction time is eliminated in this situation and a distance controller regulates the distance to the preceding vehicle. Therefore, interaction between the driver and ACC as well as the impacts on the traffic have been extensively investigated, see e.g. Davis (2004), Klunder et al. (2009), Pauwelussen et al. (2010) and Tapani (2012). 


\section{Stop \& Go}

This system is an evolution of ACC aimed at controlling the speed in slow and congested traffic. Low speed dense traffic is a complex traffic situation and therefore requires more exact estimations and control decisions, compared to ACC that is designed for high speed. The system warns the driver when the preceding vehicle is stopping and it will automatically stop the vehicle if the driver doesn't. Various Stop \& Go systems have been developed. For example, differences include the range of speed where the system can work, deceleration limit, capability, and standstill time.

\section{Cooperative Adaptive Cruise Control}

Cooperative Adaptive Cruise Control (CACC) is a further development of ACC that uses vehicle-to-vehicle communication in order to find a smaller safety distance compared to ACC. A radar measures the distance to the leading vehicle and the relative velocity. In addition, via communication with an information central, the system is able to decrease the safety distance between the vehicles. Sources of information such as GPS or a regional data centre that transmit upto-date data to road users, have been used in order to identify the speed limit or the vehicles position on the road.

The aim of CACC is to improve traffic flow efficiency and capacity, which may be achieved by smoother traffic flow, especially in merging situations. Decreasing the distance between the vehicles can lead to reduction of drag force and in turn fuel consumption. This effect is more remarkable for heavy vehicles, according to Van Arem et al. (2006). CACC has been used in platooning systems for heavy vehicles. Furthermore, it has been concluded that added inter-vehicle communication enables braking actions to reduce shock waves in traffic, but performance is affected by wireless faults and depends on market penetration of controlled vehicles.

\section{Eco Driving Cruise Control}

Eco Driving Cruise Controls are designed for exploiting the vehicle's current velocity and acceleration capabilities in order to avoid heavy acceleration and thereby increased fuel usage. It is well-known that fuel consumption is 
influenced by driving behaviour. Different drivers can obtain different fuel consumption for the same car and road. A general suggestion for driving economically is to drive slower but there are several factors that are essential in that context. Ericsson (2001) claims that energy consumption can be affected by powerful acceleration, large power demand and high engine speed. Accordingly, it has been attractive to develop systems that can optimize energy consumption, both from an environmental and an economical point of view.

\section{Down Hill Speed Control}

Down Hill Speed Control (DHSC) is designed for heavy vehicles and trucks in order to avoid exceeding a certain speed downhill. Generally, heavy vehicles have some kind of auxiliary brakes, in addition to a normal brake system. Due to large mass in heavy vehicles, the vehicle accelerates downhill if brakes are not applied. DHSC is developed to control the speed downhill by interacting with the cruise control system in the vehicle and wheel brake, which affords an optimal speed control. Activation of the DHSC is initiated by the driver and a maximum speed is fixed. This speed control system has also been called retarder.

\subsection{The Look Ahead Cruise Control}

The Look Ahead Cruise Control (LACC) is an Eco Driving Cruise Control, developed by the truck manufacturer Scania. LACC is an example of ADAS with the potential of reducing energy and emissions by controlling the speed based on the road slope, engine power and the vehicle's mass. LACC is based on what is presented in Hellström (2010) and has been further developed by Scania. LACC is designed for trucks and heavy vehicles where the road topography has considerable influence on the motion of the vehicle. Heavy vehicles have low engine power compared to their mass and the potential to save fuel by the LACC mainly depends on the slope of the road. The vehicle's position is identified by e.g. GPS and the LACC calculates the vehicle's acceleration based on a look-ahead horizon.

For the transport companies and the truck owners, truck costs are a significant part of transport costs. For example fuel costs for a class 8 truck, weighing more 
than 15 tons, are about 1/3 of the life cycle cost Hellström (2010). Load characteristics, travel distance, truck configurations, the road geographical characteristics and driving practices influence the truck costs. Many investigations have been made to improve these characteristics in order to achieve optimal energy consumption.

Figure 2.1 shows a schematic view of a hilly road section and how the LACC works based on the road slope. The truck with activated LACC enters a hill with high engine power and then starts decelerating gently and uses all the force that the engine can deliver. Downhill, the aim is to avoid braking that results in more energy use since an acceleration is required in order to achieve the desired speed after braking. The LACC reduces the speed before entering a downhill, in order to avoid retarding in a following downhill section. The speed regulation can reduce the travel time uphill and reduce fuel consumption downhill.

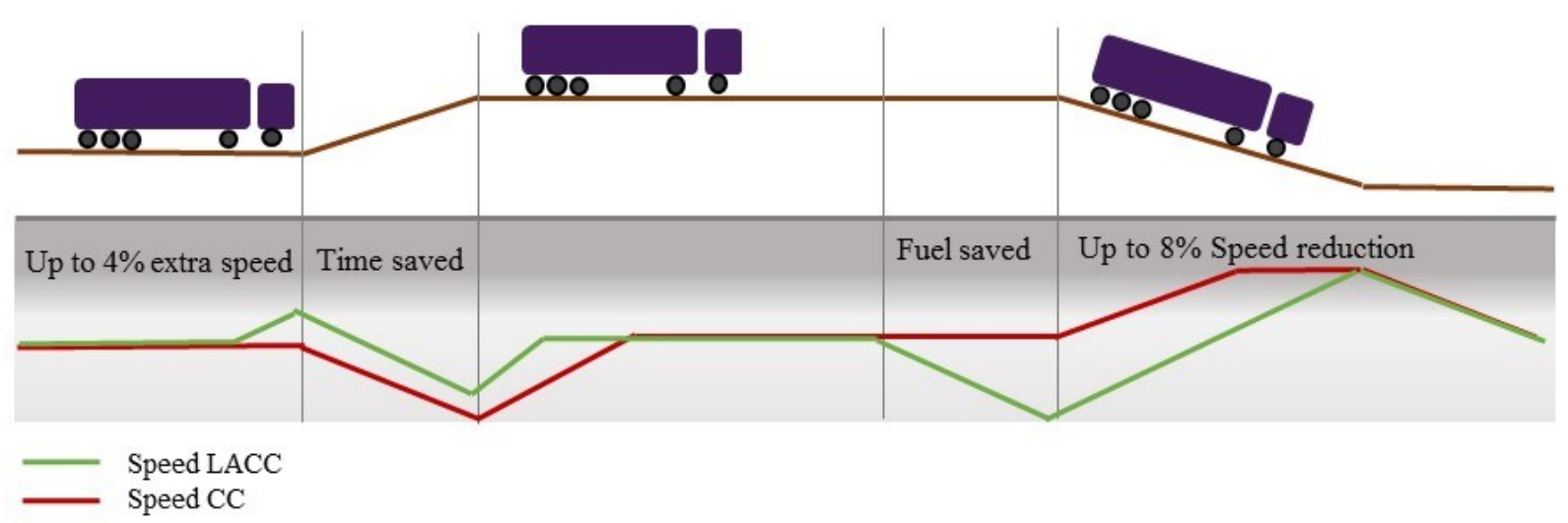

Figure 2.1. Comparison between the LACC and the CC

In other words, the LACC reacts as a skilled driver who doesn't drive aggressively and has knowledge about the road topography. Consequently, fuel consumption will be less sensitive to how experienced the driver is. However in real traffic, depending on the traffic situation, the driver has to deactivate LACC some times and drive manually, e.g. in order to avoid collisions with preceding vehicles. 
A dynamic control algorithm computes fuel-optimal solutions in LACC. The result from the algorithm is the input data for estimation of fuelling, braking and gear choice as a function of speed, gear, current position and altitude.

\subsection{Impacts of ADAS}

Investigations on ADAS impacts, have in general been utilized for various purposes. A variety of frameworks and approaches can be found in the literature. Depending on the aim of the study, expected impacts of ADAS have been identified and qualitative and quantitative measures have been investigated. Developing ADAS requires both technical modelling and human based analysis since the execution of the ADAS depends both on the technical functionality and the interaction between the driver and the ADAS. Technical modelling such as throttle and speed control as well as drive-line control can be integrated with radars, sensors or GPS to improve vehicle dynamics performance, fuel economy and reducing the environmental effects.

Minderhoud (1999) has categorized the impacts of driver support systems into user, network and environmental impacts, which represent the different directions for ADAS evaluation in many studies. This subdivision is based on driver support system impacts with respect to the driver and the vehicle, which in turn results in improvement of the driving. Figure 2.2 shows the relations between the various factors that are influenced by ADAS. According to Minderhoud (1999), when an ADAS affects the driver and the vehicle, this will influence three elements of a traffic system which are the users, the network, and the environment. This effect schematic is shown in the figure.

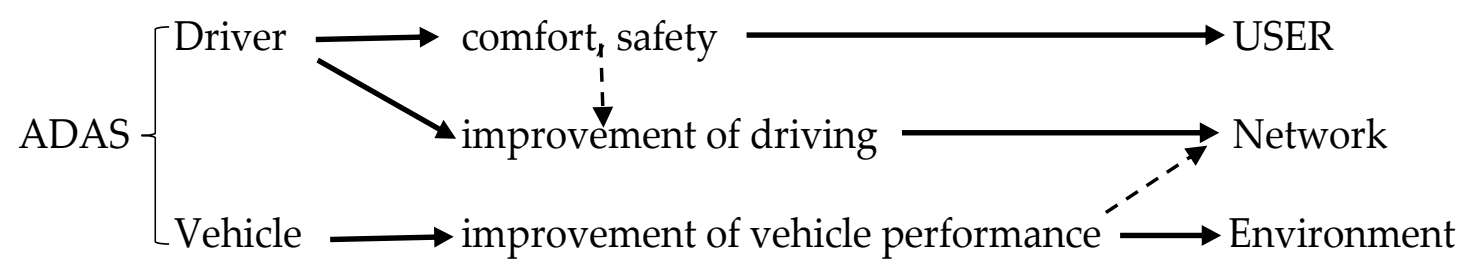

Figure 2.2. ADAS impacts as presented by Minderhoud (1999) 
During recent years, complexity of ADAS has increased and ADAS impacts with various characteristics are influenced by this escalation. Therefore, an improvement of the structure presented in Figure 2.2, is necessary for ADAS impact assessment. In this section, we present a structure for ADAS impacts based on the impacts on the driver, the vehicle, and the traffic system. This subdivision of ADAS impacts is illustrated in Figure 2.3. The effects of an ADAS on traffic are generally related to the following aspects:

- Safety

- Efficiency

- Environmental effects

In this thesis, the main focus is to investigate the potential benefits of ADAS on traffic systems, and ADAS impacts are further described in terms of safety, efficiency, and environment.

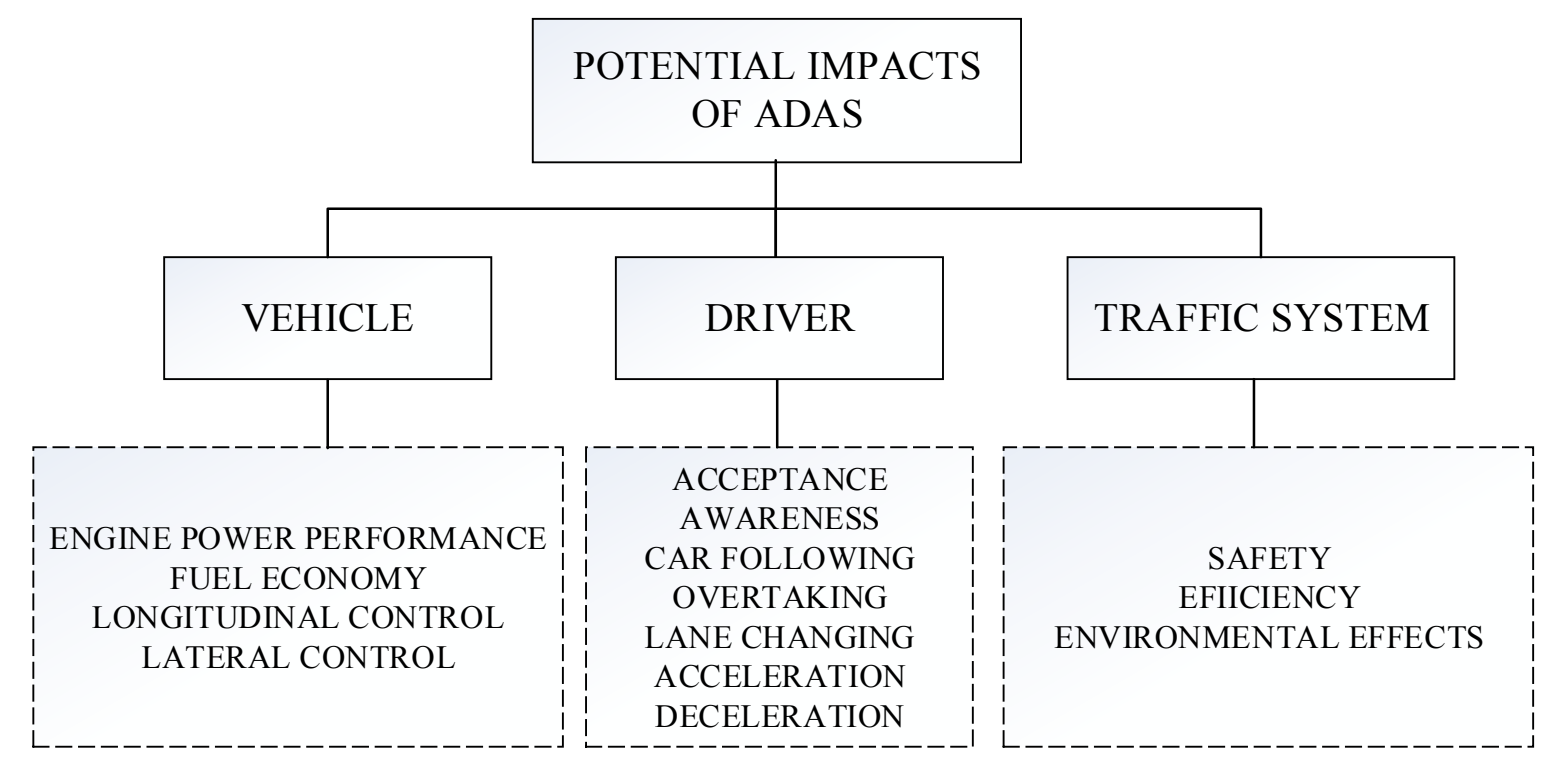

Figure 2.3 Potential impacts of ADAS

\subsubsection{ADAS vehicle impacts}

A general validation methodology for in-vehicle applications impact was developed by the CONVERGE project and presented by Maltby et al. (1998). This approach provides an overall view of the evaluation and validation process 
in transport technologies. The evaluation process involves the steps shown in Figure 2.4:

$\underset{\text { specifications }}{\text { Functional }} \rightarrow \underset{\text { specification }}{\text { Technical }} \rightarrow \underset{\text { mechanism }}{\text { Function desion } /} \rightarrow \underset{\text { Component level }}{\text { Verification }} \rightarrow \underset{\text { Expected impacts }}{\text { Hypotheses }} \rightarrow \underset{\text { Impacts }}{\text { analysis }} \rightarrow \underset{\text { Function level }}{\text { Validation }}$

Figure 2.4. CONVERGE, the evaluation process for in-vehicle applications

The basic principle of technical evaluation of ADAS is to verify if the system works as required, and this methodology has been the basis for many ADAS evaluations from a technical point of view.

A workflow with six evaluation steps is presented by Scholliers et al. (2011) which is based on the evaluation process in CONVERGE:

1. Functional specification with a detailed functional description including all assessments and functions sketches to assure that all needed information is available. The main purpose of this step is to prepare functional verification of the system which requires detailed knowledge about sub-systems and components. This step provides the limitations and potential improvements for the complete system according to the functional specifications.

2. Expected impacts will be identified and described at a sufficient level of detail in order to validate the performance of the system. Various indicators can be used for assessment of potential improvement by the system, e.g. time to collision, missed alarm rate (MAR) or false alarm rate (FAR). The FAR and MAR improve the precision of an alarm system in detecting risky situations correctly.

3. Scenario definition contains descriptions for different situations for which the system is designed. Various operational conditions should be tested in this step to verify if the system executes correctly. 
4. Method selection depends on the functionality of the system and purpose of the evaluation. Field Observation Tests (FOT), driving simulators, vehicle simulations, traffic simulations and hardware-in-the-loop test are examples of evaluation methods.

5. Test plan specifies the variables and measurements needed for sampling. The important aspects of the ADAS functionality and impacts should be included in the test plan and all influenced factors should be taken into account. The experimental design should include number of participants to statistically significant results.

6. Execution and reporting includes running tests, analysing and reporting conclusions. A recognized issue in ADAS evaluation is how to design tests and analyses on ADAS impacts when other ADAS are active simultaneously.

A further step in evaluation process is to identify and verify the systems functionality with respect to the vehicles safety, efficiency and environmental effects.

\section{Safety impacts of ADAS on the vehicle}

A basic principle of ADAS evaluation is to identify the potential elements in the vehicle that can be affected by the ADAS. Implementing ADAS in a vehicle involves elaboration with sensors, microelectronic controllers and radars for driving assistance. However, ADAS might affect the equipped vehicle in various ways. The system should be reliable and function properly. Components in ADAS have quite different functions in a vehicle. Testing these components requires dynamic algorithms in order to investigate how ADAS will influence the other systems and vehicle dynamics. In other words, the safety implications of ADAS on the vehicle, involve the functional safety issues such as hardware and software design. 


\section{Efficiency impacts of ADAS on the vehicle}

Vehicle performance, energy consumption and vehicle dynamics (longitudinal and lateral) are most mentioned impacts in the literature. However, it is essential to study ADAS efficiency based on the aim of ADAS.

\section{Environment impacts of ADAS on the vehicle}

The problem of environment pollution by transportation has been demanding more attention on fuel control systems. In-vehicle systems have been developed in order to optimize engine, motor and battery efficiency. In addition, enhanced communication between vehicles and infrastructure can decrease the environmental effects both for the individual vehicle and the traffic system.

\subsubsection{ADAS impacts on the driver}

Many ADAS influence driving behaviour, which can determine how useful and effective the system is. For example, it is important to observe how willing drivers are to use an ADAS and how drivers are able to interact with the system.

A human driver interacts with ADAS in many traffic situations, with reflection about the surrounding vehicles and the vehicle position, speed etc. Since ADAS is designed to inform or support the driver in some driving tasks, it is expected that drivers take over vehicle control based on the situation, if needed. Measurements of human factors are often difficult to combine with functional variations. However, the interaction between the driver and the ADAS at different levels of automation is worth noting. Understanding the information presented by the ADAS as well as the performance of the actions using the ADAS, affects the final execution of the system. In order to integrate ADAS with other systems in the vehicle successfully, it is important to understand and estimate variations in driving behaviour and acceptance when using an ADAS.

\section{Safety impacts of ADAS related to the driver}

One of the challenges in ADAS evaluation is to predict the safety impacts of ADAS. A reliable and realistic evaluation should anticipates human behaviour while driving with an ADAS equipped vehicle in different traffic situations. 
According to AIDE D1.1.4 (2004), Information Processing Systems (IPS) have been applied in most driving behaviour models in order to describe the interaction between the driver and in-vehicle control systems. IPS consists of the following steps based on cognitive and behavioural interactions:

- Observation and understanding information from the vehicle and the traffic

- Decision and selection of tasks to execute

- Performance of actions in vehicle control

In other words, the interaction between the driver and the ADAS is important from a safety point of view. van Driel (2007) also mentions mental workload caused by using ADAS as a safety issue. It is important to verify if the system can be used safely without too much new mental workload.

\section{Efficiency impacts of ADAS related to the driver}

Changes in driving behaviour caused by ADAS are first of all, related to the drivers' willingness and attitude to use the ADAS. The drivers' expectations of the system and the unwanted changes in driving behaviour can also influence the perceived benefits of the ADAS.

Measures of driving behaviour can be used to evaluate driving performance. According to AIDE (2005), these measures can be distinguished in three categories:

- Longitudinal control measures such as mean speed, maximum speed, mean acceleration, and mean distance headway ${ }^{1}$.

- Lateral control measures such as standard deviation of steering wheel angle, mean lateral position, lane changes, and line crossing.

\footnotetext{
${ }^{1}$ The distance headway is defined as the distance between corresponding points of two successive vehicles at any given time.
} 
- Event detection measures such as response time ${ }^{2}$, response distance, errors of omission $^{3}$, and errors of commission ${ }^{4}$.

These measures can be used in order to verify the efficiency of an ADAS in interaction with the driver.

\section{Environmental impacts of ADAS related to the driver}

Before or during driving, a driver may be better prepared with knowledge about the traffic condition or congestions. An ADAS that informs the driver about traffic conditions and supports the driver in planning the trip more effectively can influence the environmental effects of driving. If an ADAS provides smoother accelerations and deceleration or uses technologies for enhanced engine power performance, it will decrease the energy consumption and pollution. The ADAS can compensate the drivers' errors that are related to experience, characteristics, gender, culture, etc.

\subsubsection{ADAS impacts on the traffic system}

Many ADAS have been developed in order to achieve a more efficient, safer and environmental friendly traffic system. A central discussion in ADAS impacts evaluation is to determine how an ADAS will affect the traffic, from an aggregated point of view. ADAS impacts on vehicles and drivers have already been discussed in numerous studies and a further step is the analysis of the ADAS effects on traffic systems.

\section{Safety impacts of ADAS related to the traffic system}

Increased traffic safety requires reduced conflicts and accidents in traffic. Many traffic accidents are caused by human driver errors and various ADAS have been developed in order to compensate these errors. The safety potential of an

\footnotetext{
${ }^{2}$ Response time/distance is the time/distance a system or functional unit needs to react to a given input.

${ }^{3}$ An error of omission is an error that occurs when action has not been taken or when something has been left out.

${ }^{4}$ An error of commission is a mistake that consists of doing something wrong, such as including a wrong amount, or including an amount in the wrong place.
} 
ADAS can be determined based on several factors. However, the number of accidents or conflicts is the most common measure of traffic safety. In order to identify the ADAS safety impacts, technical performance of ADAS in real traffic and interaction between the driver and the system are commonly considered. Variations in speed, acceleration, braking, headways and lateral position of the vehicle can monitor the safety impacts of an ADAS. For example, the acceleration and hard braking distribution can indicate how smooth a traffic flow is. Moreover, exceeding minimum safety headway or increased headway between vehicles can show impacts on traffic safety. Occurrence of unexpected changes in lateral position or keeping the vehicle in the same lane can also be observed. Conflicts are one more key measurement in traffic safety. A traffic conflict has been defined as 'An observable situation in which two or more road users approach each other in time and space to such an extent that there is risk of collision if their movement remains unchanged' by Amundsen et al. (1977).

Generally, variations in speed and acceleration, time headway and time to collision (TTC) as a result of driving with ADAS have been measured as indicators for longitudinal control. Headway is a key indicator in assessing traffic safety and capacity. Time headway is defined as the time it takes between the leading vehicle and following vehicle to reach the same point on the road. In other words, time headway is the time it takes between two vehicles to pass a specific position on the road. Larger headways imply more space between the vehicles in the traffic that obviously affect the number of conflicts. Time headway has been used to estimate how critical a traffic situation is.

Safety evaluation of ADAS requires considering changes in driving behaviour as well as safety measures. For instance, if the ADAS has a longitudinal control function, the safety measure can be based on longitudinal safety indicators such as time-to-collision (TTC) or utilized deceleration rate.

Road safety is an important topic in many countries and it has traditionally been measured based on statistical models and analysis of the actual accidents. These analyses are conducted reactively based on historical data and measurement of the level of unsafety on the road, such as number of accidents and casualties. In many studies, safety performance indicators have been used as quantitative or qualitative measures that are gathered from historical data and facts. 
Time to Collision (TTC) is relevant to calculate if the following vehicle has higher speed than the vehicle in front. The TTC is the time that it takes for two vehicles to crash if they continue with same speed, and it can be expressed as:

$$
\operatorname{TTC}_{n}=\frac{x_{n-1}(t)-x_{n}(t)-l_{n}}{v_{n-1}(t)-v_{n}(t)} \quad, \forall v_{n-1}(t)<v_{n}(t)
$$

Here $x_{n}(t)$ is the position of vehicle $n$, and $v_{n}(t)$ is the speed of vehicle $n$, at time $t$.

TTC has often been used as a safety indicator for certain situations, for example when some vehicles in the traffic are controlled by different in-vehicle systems.

Distance headway is defined as the distance between the leader and the follower at any given time. Measures such as lateral position and lane exceeding have been utilized for example, to determine accident probability. For event detection systems, it is common to create sudden changes around the vehicle and observe the systems response. Situations such as when the preceding vehicle suddenly brakes or when a pedestrian or obstacle appears, can be different scenarios for studying the system reaction.

\section{Efficiency impacts of ADAS related to the traffic system}

Numerous investigations have been conducted to develop and evaluate ADAS that make the traffic flow more efficient and relieve traffic jams. Minimizing congestions and more effective traffic flow increase traffic performance, which have many well-known benefits, such as reduced costs and air pollution and increased level-of-service.

Traffic efficiency is a macroscopic characteristic performance. The most common measures that have been used for investigation of traffic efficiency are traffic flow, density, speed, travel time, delay, acceleration distribution, and traffic breakdowns.

Traffic breakdowns and congestions are probably the most studied characteristics of traffic efficiency. Distribution of vehicles over the lanes, speed variations and number of accelerations and braking are indicators that show whether the traffic is smooth. 
Changes in road capacity and throughput are commonly measured in order to verify if an ADAS has impacts on traffic efficiency. Increased traffic flow and speed in general, indicate an improved traffic performance. In addition to ADAS impacts on traffic efficiency, different penetration rates of ADAS in the traffic can be investigated. For example, the penetration rate of the ADAS that have congestion assistance functions, influences congestion positively, according to Van Arem et al. (2006).

Kesting et al. (2007) have studied different levels of ACC equipped vehicles in traffic and state that increased penetration rate of ACC equipped vehicles can lead to increased road capacity.

\section{Environmental impacts of ADAS related to the traffic system}

The energy and emission rates in traffic are influenced by many factors and evaluation of ADAS with respect to environmental effects has been conducted in different ways. In order to achieve reliable and realistic evaluation results, it is important to take into account as many factors as possible.

The demand for transport is the main factor influencing air pollution. Mode choice, fuel quality, road conditions and driving style are the other factors affecting the environment. Reducing the environmental effects requires considerations and improvements in all these factors. The energy and emission usage are influenced by many factors. According to Ahn et al. (2002), six broad categories, can be identified as:

1) Travel-related: mode choice, distance, number of trips and driving hours

2) Weather-related: temperature, wind and air resistance, humidity

3) Vehicle-related: Engine power, mass, whether the vehicle is equipped with energy reducing systems, energy saver tires, etc.

4) Roadway-related: curvature, geometry and roadway surface roughness

5) Traffic-related: traffic density and congestions, number of decelerations and acceleration, idling, etc.

6) Driver-related: age, cultural climate, lifestyle, gender, driving experience, driving style, etc. 
Chapter 2. Advanced Driver Assistance Systems (ADAS) 


\section{Chapter 3}

\section{ADAS evaluation}

This chapter presents methods and tools that have been used to assess ADAS impacts. As the main evaluation method used in this thesis is microscopic traffic simulation, we introduce traffic simulation in Section 3.2, and Section 3.3 reviews earlier research that has used traffic simulation for ADAS evaluation. The findings of this chapter is used as a basis for the design of a combined evaluation framework presented in Chapter 4.

\subsection{Evaluation methods}

Numerous studies have been conducted to identify ADAS impacts. The main questions have been either the verification of the ADAS' functionality, integration between ADAS and other systems in the vehicle, interaction between ADAS and the driver or the interaction between the ADAS-equipped vehicle and other vehicles. Development of ADAS has been concentrated on the technology, but the importance of the driver's acceptance and interaction with the system is more evident now. This trend is also taken up in the ADAS 
evaluation methods. Recently, the effects of ADAS on the driver and driving behaviour have been examined in several studies. Although these systems are designed to assist the driver, they might lead to distraction, human errors and information overload, or startling the driver. This knowledge is further indicated by many researches that provide insight of interaction between the driver and ADAS.

The general objectives of an ADAS are basically designed by the manufacturers. However, how the drivers accept and use these systems affect system usability. For instance, if an ADAS is designed to increase the comfort level of driving or to improve longitudinal movement, the final execution of ADAS also depends on the system interaction with the driver. A driving assistance system affects the driving process, which should be evaluated from a variety of detailed measurements of the observed vehicle such as driving behaviour, speed, braking, acceleration, lane position or distance keeping. One of the challenges in ADAS evaluation is to predict behavioural impacts and interaction between the driver and the ADAS. In addition, attaining enhanced impact estimations of ADAS both on the equipped vehicle and the surrounding traffic is essential. Correct vehicle dynamics modelling is more implied in powertrain-related ADAS, because the changes in the vehicle dynamics (caused by the ADAS) influence the equipped vehicle, as well as surrounding vehicles.

Different tools can be used to evaluate ADAS. Therefore, it is important to know the ADAS function, purpose of evaluation and the abilities of the proposed evaluation tool. In order to obtain reliable evaluation results, it is essential to identify the target measurements, requirements, hypotheses, capacity and limitation of ADAS as well as the evaluation tool. Most common ADAS evaluation tools are:

- Field Observation Test

- Questionnaires / Focus Group

- Driving simulators

- Vehicle simulation models

- Traffic simulation models

- Fuel and emission models 
This section describes these evaluation methods for exploring the related capabilities and limitations.

\section{Field Observation Test}

A Field Observation Test (FOT) observes a real driver in real traffic conditions. FOT is one of the evaluation procedures commonly used for driver assistance systems, which examines hypotheses in real world conditions. The idea is to construct the experiment in a naturally occurring environment without laboratory-controlled settings. The FOT method is generally used in a late stage of the development, when it is possible to apply the system in real world conditions. The aim of a FOT is to evaluate the interaction between a system and the real environment and to provide further understanding of people's behaviour and needs. However, a weakness is that a FOT study cannot be reliable especially in the early stage of ADAS deployment, due to the proportion of equipped to unequipped vehicles in the real traffic, Tapani (2011).

\section{Questionnaires / Focus Group}

Data collection using questionnaires or focus group studies are suitable methods for the non-technical analysis such as drivers' acceptance and behaviour. These methods are based on qualitative data analysis revealed from discussions in the focus groups or questionnaires. Holtl et al. (2013) have discussed different ADAS applications and user acceptance with the empirical data, in order to show the differences and interrelation of acceptance factors: perceived usefulness, perceived ease of use, changed driving behaviour, and perceived efficiency. This kind of assessment is crucial for designing and evaluating ADAS since the effectiveness of the system depends on the users' acceptance.

\section{Driving simulator}

Testing drivers' behaviour in a laboratory situation using a driving simulator that includes implementation of ADAS functionality is a common tool for studying the impacts of driving assistance systems. The advantage of using the driving simulator is the possibility to control traffic conditions, however, the weakness can be the validity of the simulator. 
Driving simulators are often used to evaluate a new in-vehicle application or a change in infrastructure. By putting the driver in a desired virtual traffic situation it is possible to observe driving behaviour. One of the advantages of driving simulators is the ability to study various traffic situations. It is also possible to accomplish the same traffic situations for different drivers. Another strength of the driving simulator is the possibility to provide traffic situations that seldom occur in real traffic.

A scenario in a driving simulator experiment includes descriptions of road geometry and curvature, number of vehicles in the network during the experiment, penetration rates of road users and variations in vehicles, type of road, weather conditions, road surface, etc. Furthermore, simulator models implement vibration, noise and graphics in order to minimize differences between the simulator and the vehicle. Due to technical limitations in driving simulators, it is often necessary to filter some input data such as road surface data, the size of the roads longitudinal profile, weather conditions, etc.

\section{Vehicle simulation}

Vehicle simulations are used, for example, by vehicle manufacturers to develop and evaluate new components and concepts, to tune parameters, and to conduct verifications before real-world testing. Development of vehicle technologies, i.e. powertrain, chassis- and controller design, active fuel systems and driving assistant systems, involves a wide range of evaluations and analysis. Vehicle dynamics simulations are essential tools for validating architectures and models before applying them on the vehicles. These simulation models are based on a mathematical representations of the physical systems in vehicles. The models assist vehicle dynamics engineers in designing and further development of the systems that will be included in a vehicle. A road description and driver model are commonly used as input. The driver model often consists of predefined driving trajectories for a limited set of relevant traffic situations and scenarios.

\section{Traffic Simulation}

Microscopic traffic simulation is usually used to estimate the level of service of various types of infrastructure and traffic control designs (see e.g. Barceló 
(2010)), but it can also be used to study ADAS effects on traffic. Driver behaviour sub-models are used to model the various parts of the driving task, such as car following, speed adaptation, lane changing, merging, and gap acceptance (see Olstam et al. (2008) for an overview).

Nowadays, conducting road safety analyses by microscopic traffic simulation with capability to model the individual drivers, has realised more attention. For example, without waiting for a real accident in traffic, it is possible to develop safety indicators with microsimulation software.

Further descriptions about traffic simulation models are available in Section 3.2.

\section{Fuel and emission model}

The environmental effects of road transport are commonly evaluated by fuel and emission models. Emission models are deigned to estimate pollutant emissions caused by road transport, which depend on many factors. Emission factors are empirical functional factors that estimate relations between emissions and the activity that causes them. Such factors are usually calculated based on experimental data for vehicle categories. Many parameters such as emission control technology, the vehicles weight, engine power and operating conditions may affect the calculation of emission factors in an emission model.

Vehicle emission models can be categorized based on the level of complexity and range of application, in microscopic and macroscopic emission models. Macroscopic emission models estimate the emissions as a function of mean travel speed. In microscopic emission models, emission rates correspond to the engine or vehicles operating conditions or a specific traffic situation, for example free flow or on ramp situations. The emission models that estimate emissions based on average speed or traffic situations are suitable for emission estimation at national or regional level. Modal emission models with calculations based on vehicles characteristics are designed for local and small networks. PHEM (Hausberger 2010), CMEM, (Scora et al. 2006) and MOVES (Koupal et al. 2003) are examples of modal emission models. Based on the vehicles longitudinal dynamics in combination with road gradients, the emission model calculates the engine power output for the average vehicle groups. The vehicles longitudinal dynamics can be the outcome from a traffic 
simulation experiment. A database for the average vehicles is generally used in emission models. The database is developed based on calculating emissions of different vehicle groups. The vehicle trajectories received from the traffic simulation experiment can be used as input to the emission model, and the energy consumption and the traffic related emissions can be calculated by the emission model.

\subsection{Traffic simulation}

Traffic simulation models are usually categorized as macroscopic, microscopic or mesoscopic, according to the level of data in the model. Macroscopic simulation models manage traffic flows from an aggregated point of view with variables such as density, volume and speed. Microsimulation models are based on individual vehicles dynamics indicating acceleration, deceleration, lane change etc. and is often applied in relatively small networks. The possibility of observing individual vehicle dynamics in interaction with driving behaviour renders microscopic simulation more suitable for evaluation of ADAS impacts. Mesoscopic simulation models represent a modification of the microscopic models, with simplifications of the vehicle dynamics. By capturing the most essential variables in the vehicles movements, mesoscopic models are more time efficient than microscopic models, Barceló (2010).

The evaluation platform used in this thesis is based on microscopic traffic simulation and therefore, this section gives a description of such method. Microscopic traffic simulation models can be used for analysing various traffic operations such as signal operations, planning of public transport, managing traffic congestions and merging areas.

The microscopic traffic simulation models consist of several sub models. During a simulation, the vehicles in the simulated road are updated according to vehicle behaviour models. A microscopic traffic simulator consists of two main features, Hengst (2010):

- The traffic flow simulation model including the models for car following, lane changing, overtaking and gap acceptance. 
- The traffic control model that manages detector information in the traffic network.

The car-following and lane-changing models are the essential sub-models in a microscopic traffic simulation model. Therefore, we introduce theses submodels in the next sections.

\subsubsection{Car-following models}

The car-following model is the most crucial sub model that operates the longitudinal interaction with the preceding vehicle. Some car-following models are based on the follower's velocity in relation to the preceding vehicle, and other models also take into account acceleration or the distance between the vehicles. Three types of car-following models are described by Hoogendoorn et al. (2001):

- Safe-distance models

- Stimulus-response models

- Psycho-physical models

Safe-distance models describe how a vehicle adjusts movement in relation to the preceding vehicle in order to not traverse a (specified) minimum distance headway. A first simple safe-distance model was presented by Pipes (2004) as

$$
D_{n}(v)=L_{n}(1+v / 16.1),
$$

Where $D_{n}$ is the required distance headway, $L_{n}$ is the vehicle length $n$, and $v$ is the relative velocity according to preceding vehicle $n-1$. The safe distance is linearly depended on the vehicle's velocity. Enhanced safe-distance models are considering the perception time, decision time and braking time as well.

(Gipps 1981) model is probably the most well-known safety distance model. The model calculates the maximum speed that the follower vehicle can reach during the time period $(t, t+\theta)$. The follower's speed at time $t+\theta$ is influenced by the desired speed that the vehicle tries to achieve according to

$$
v_{n}(t+\theta)=v_{n}(t)+2.5 a_{n}^{\max } \cdot \theta \cdot\left(1-\frac{v_{n}(t)}{v^{d e s}}\right)\left(0.025+\frac{v_{n}(t)}{v^{\text {des }} n}\right)^{1 / 2}
$$


Furthermore, the follower's speed is limited by the leading vehicle's speed, position and desired deceleration, which can be expressed as

$$
\begin{gathered}
\bar{v}_{n}(t+\theta)=\theta \cdot d^{\text {des }}{ }_{n}+\left(\theta^{2} \cdot d_{n}^{\text {des }}{ }^{2}-\right. \\
\left.d_{n}^{\text {des }} \cdot\left[2 \cdot\left(x_{n-1}(t)-l_{n-1}(t)-x_{n}(t)\right)-\theta \cdot v_{n}(t)-\frac{\left(v_{n-1}(t)\right)^{2}}{d^{d e s} s_{n-1}(t)}\right]\right)
\end{gathered}
$$

Where $v_{n}(t)$ is the speed, $x_{n}(t)$ is the position and $v^{\text {des }}{ }_{n}(t)$ is the desired speed of the vehicle $n$ at time $t$. $a_{-} \max _{n}$ is the maximum acceleration for the vehicle $n$, $\theta$ is the reaction time and $d^{\text {des }}{ }_{n}$ is an estimated desired deceleration for the vehicle $n$. For vehicle $n-1, l_{n-1}$ is the effective length, that is the vehicles physical length plus a minimum distance that the follower is not willing to enter.

(Gipps 1981) stated that the speed of the following vehicle $(n)$ during time interval $(\mathrm{t}, \mathrm{t}+\theta)$ is the minimum of $\bar{v}_{n}(t+\theta)$ in (2) and $v_{n}(t+\theta)$ in (1):

$$
V_{n}(t+\theta)=\min \left\{\bar{v}_{n}(t+\theta), v_{n}(t+\theta)\right\}
$$

Stimulus-response models are based on a general assumption that drivers try to imitate the driving behaviour of the preceding vehicle:

$$
\text { Response=sensitivity } \times \text { stimulus }
$$

The response is the acceleration or deceleration of the follower, which is delayed by a reaction time.

The Gazis-Herman-Rothery (GHR) category of the car-following models are probably the most studied category and it specifies acceleration as

$$
a_{n}(\mathrm{t}+\mathrm{T})=\alpha \cdot v_{n}(\mathrm{t}+\mathrm{T})^{\gamma} \cdot \frac{\left(v_{n-1}(t-T)-v_{n}(t-T)\right)}{\left(x_{n-1}(t-T)-x_{n}(t-T)\right)^{\beta}}
$$

Where $v_{n}(t)$ and $a_{n}(\mathrm{t})$ are the velocity and the acceleration of vehicle $n$, and $\alpha>0, \beta$ and $\gamma$ are the parameters that indicate the drivers sensitivity. This means that the following vehicle's decision to accelerate or brake, depends on both the distance and relative velocity between leader and follower. 
Psycho-physical models use action points or thresholds to classify different regimes in driving. Wiedemann Wiedemann (1974) developed the first psychophysical model. The psycho-physical models use action points or thresholds where the driver changes driving behaviour, according to space between the vehicles or the relative velocity. In other words, a faster vehicle behind a slower vehicle in the same lane starts to decelerate as the threshold is reached. The suggested driving regimes are:

Free Driving: when there is no observable influence of the preceding vehicle and the driver seeks to reach the desired speed.

Approaching: In order to adapt speed to the preceding vehicle with lower speed, the driver decelerates to get the relative speed as near to zero as possible.

Following: Keeping the safety distance is the action here, without any conscious reaction.

Braking: A substantial deceleration e.g. to avoid a collision.

The thresholds represent the individual perception and are defined in relation to the relative distance $(\Delta x)$ and relative velocity $(\Delta v)$. An illustration of different thresholds and regimes in Wiedemann's model, is shown in Figure 3.2. The figure shows that the driver is influenced subconsciously when the relative speed remains in the regime that is marked with green (FOLLOWING) and reacting when the relative speed is in the yellow regime (REACTION). 


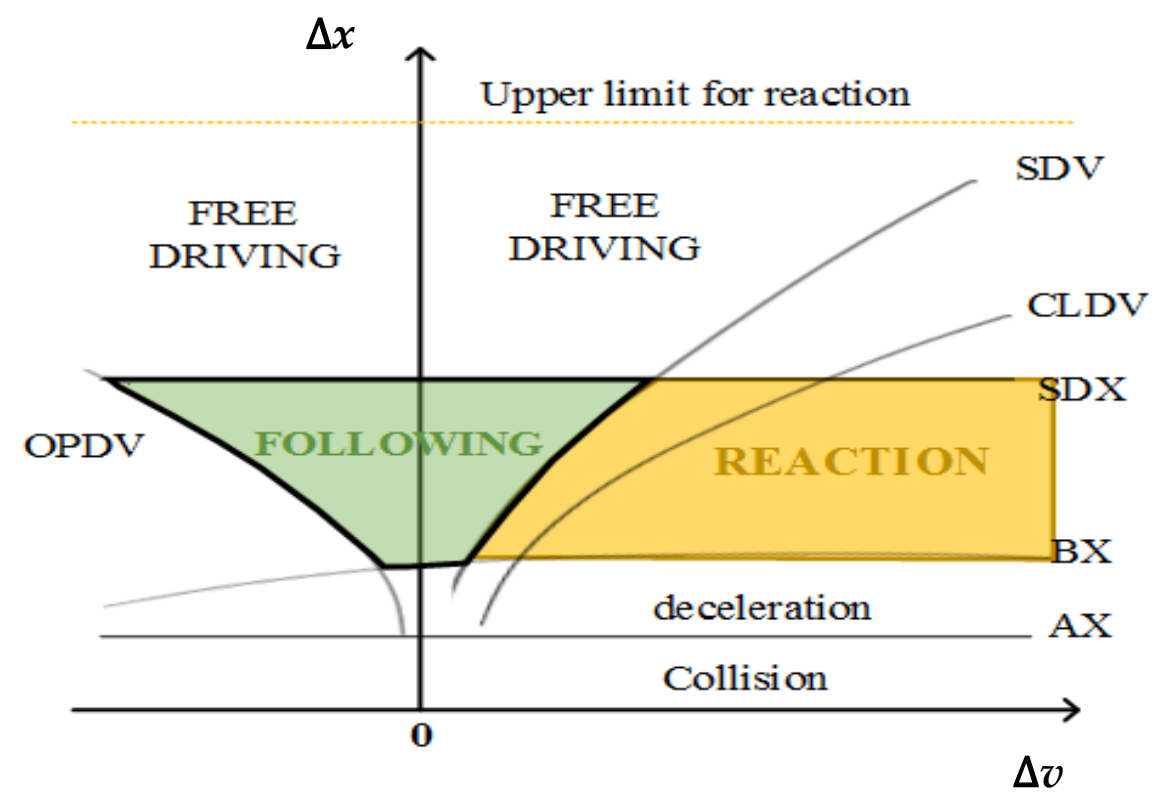

Figure 3.2. Regimes in the Wiedemann car-following model

The following regimes describe the thresholds:

SDV: Speed difference at large distances where drivers consciously realize that they are closing in on a slower vehicle, and start to reduce their own speed.

CLDV (closing $\Delta \mathrm{v}$ ): Speed differences in decreasing distances. The follower decelerates as the distance clearly decreases.

OPDV (opening $\Delta \mathrm{v}$ ): Speed differences in increasing distances. The follower accelerates as the distance clearly increases.

SDX: Maximum following distance, where the driver consciously recognizes that the vehicle is not following the vehicle in front anymore, and reacts by accelerating.

AX: The desired distance between the vehicle and the vehicle in front (in a standing queue)

BX: The desired minimum following distance at low speed differences. 


\subsubsection{Lane-changing models}

The lane changing model is another essential sub-model used in micro simulation models that simulate multilane traffic flows in freeways, highways and urban networks. Lane-changing behaviour clearly affects road safety and traffic performance and thereby many studies have been conducted in order to better understand or model this kind of driving behaviour.

Lane-changing activities consists basically of three decision or action moments: monitoring the traffic situation, vehicle control such as steering and acceleration, and decision to change lanes. Various parameters are used in lanechanging models in order to model real traffic situations and variations in drivers' decisions.

In most lane-changing models, the first question is confirming if lane-changing is necessary in order to avoid a lane obstacle, follow a planned travel route or maintain desired speed. Whether or not the target lane is appropriate or the gap between the vehicles is acceptable, are other issues in modelling lane-change behaviour. According to Gipps (1981), lane-changing models approximate the driver's decision to change lanes, based on three main questions:

- Is it necessary to change the lane?

- Is there a benefit in changing the lane?

- Is it feasible to change the lane?

Figure 3.1, shows a lane-changing situation, when vehicle no. 4 considers a lanechange manoeuvre. In this case, the driver has to decide if it is feasible to change lanes and estimate the gap between the vehicles as well as the relative velocity in the adjacent lane, before starting the lane-changing manoeuvre. In multilane roads, the target lane might be other than the adjacent one, but it requires that the vehicle moves into the adjacent lane a couple of times, until it is on the target lane. 


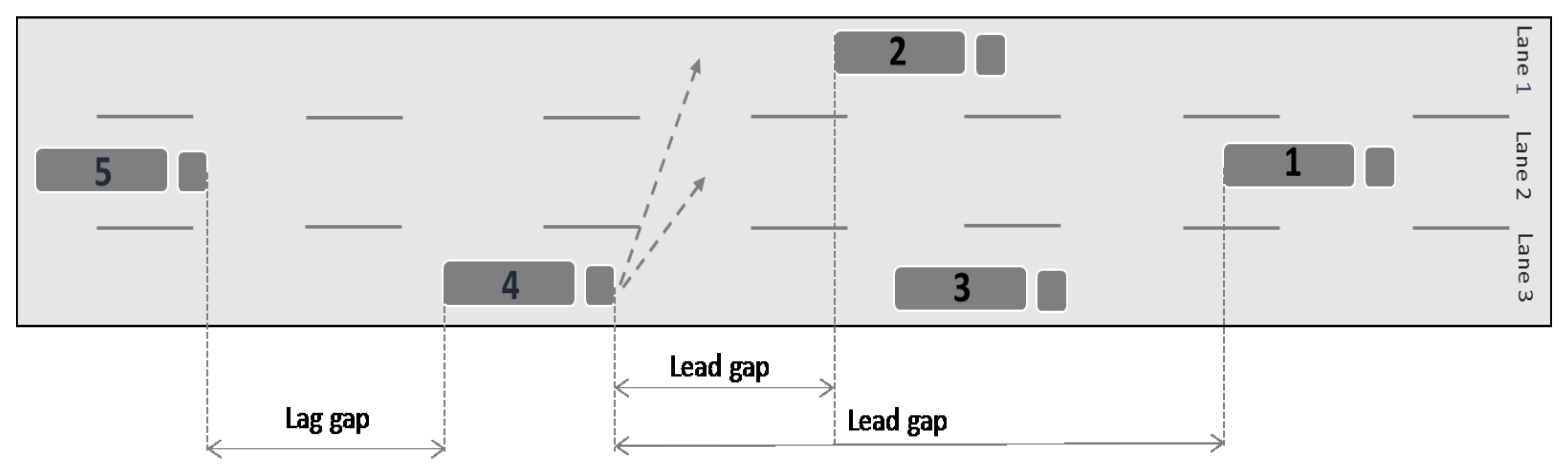

Figure 3.1 Possible lane-changing

In most of the gap-acceptance models, it is assumed that each driver has a critical gap, a minimum gap in the target lane that the driver is willing to accept in lanechanging. Critical gaps vary due to the differences in drivers' perception and traffic situations. Various gap acceptance models have been developed in order to estimate the critical gap and driver's decision. Acceleration rate, desired speed, speed acceptance and maximum give-way time are other parameters used in gap acceptance models.

\subsection{Traffic simulation of ADAS}

Traffic simulation models have traditionally been used to study impacts of road design and traffic management measures. During the last decades, the expansion of in-vehicle driver support systems has increased interest in using traffic simulation models for evaluating the impacts of these systems. Traffic simulation analysis of Adaptive Cruise Control (ACC) is the most common type of study, but other system such as intelligent speed adaption (ISA) and overtaking assistance have also been examined. However, Tapani (2011) concluded that many of these studies considered only the system functionality and not changes in driver behaviour that the system may induce. It is also clear that current state-of-the-art microscopic traffic simulation modelling excludes the driver behaviour effects of ADAS.

It is of great importance to examine the effects of ADAS at an aggregated level and several studies have been conducted. Tapani et al. (2012) presents an introduction to evaluating of ADAS by microscopic traffic simulation. The car- 
following models include the ACC's functionality and driving behaviour influenced by the ACC. The analysis is based on changes in driving behaviour such as desired speed, desired following gaps and reaction times. The vehicles' acceleration and deceleration rates are also considered in the analysis. The results show that ACC could improve traffic safety and comfort and decrease emissions, due to better distance-keeping and smoother acceleration.

Kesting et al. (2007) use microscopic traffic simulation modelling for investigating how different penetration rates of ACC-equipped vehicles influence the capacity and stability of traffic flow. The fact that traffic simulation models allow the user to specify the parameters and distributions of cars, trucks and other vehicles in traffic, is one of the advantages of these models. Moreover, it is possible to implement ACC functionality and design a traffic simulation scenario with ACC-vehicles as well as manually controlled vehicles. It is mentioned that the functionality of ACC is the same as in a car-following model and therefore driving behaviour should be taken into account. The Intelligent Driver Model (IDM) presented by Treiber et al. (2000) is used to generate a crash-free collective dynamics. Based on IDM a new car-following model has been proposed using a constant-acceleration heuristic (CAH) with safe acceleration. To analyse the outcome, the means and standard deviations of driver behaviour measurements in a matrix of transitions is considered. The results show that different conditions such as the proportion of trucks and ACCequipped vehicles and different ACC strategies lead to overruling the ACC.

A Field Observation Test (FOT) for the project Roads to the future, was conducted in the Netherlands. The idea was to combine FOT and traffic simulation to evaluate impacts of ACC. Klunder et al. (2009) present the results of this study concerning the effects of different penetration rate of ACC-vehicles in traffic. The main objective was to evaluate when drivers decide to deactivate or reactivate the ACC and the effects of this behaviour on traffic flow. Reviewing the data shows that ACC was difficult to use during congestion. The drivers regularly switched the system on and off even during busy traffic or free driving. The changes in dynamic behaviour of a vehicle equipped with ACC influence surrounding vehicles, and in turn, the traffic system will be affected. To estimate these effects for larger penetration rates, traffic simulation was used. Two scenarios of using ACC were simulated. In the first one, ACC was switched on and off, and in the second ACC was always activated. Limitations 
and characteristics in the ACC system, such as set distance and set speed were used for implementation of the system in the simulation model. A driver model was implemented using the application programmer interface in (PARAMICS 2003). The Longitudinal vehicle control in the driver model means that the driver tries to maintain the desired speed in free-driving situations and adapts the speed based on the distance to the vehicle in front otherwise.

It is assumed that a driver will control the vehicle more cooperatively than ACC in traffic, according to Klunder et al. (2009). This means that human drivers take into account surrounding vehicles driving behaviour, speed, lane-changing, etc. and therefore the ACC will be overruled due to the systems limitations. Three existing cooperative driver models are used therefor, to model the effect of the ACC system on traffic flow to adapt the related parameter in these models. The driver model was also extended by the cooperative driver models: active gap creation, active gap searching, and adaption to speed in the left lane. A mixed traffic in a highway segment is assumed, with different penetration rates $10 \%$, $50 \%$ and $100 \%$ ACC-equipped vehicles. The results show that on- and offswitching of the ACC affects traffic flow especially in congestion.

Davis (2004) studies the effects of ACC on traffic flow, especially in relation to congestion. The aim is to determine if ACC influences traffic flow in bottlenecks and on-ramp situations. ACC-equipped vehicles are randomly mixed in the simulated traffic and the formation of jams are considered. The dynamics of ACC-equipped vehicles were described as a function of the velocity, time headway, and a delay time. The effect of mixing ACC-equipped vehicles with manually driven vehicles was studied in single- and multilane simulations. The velocity of the vehicles was studied with respect to the position on the road and, for example, the distance to a merge region. The proportion of ACC-equipped vehicles show different impacts on jam building. The average velocity seems to increase and this effect was slightly more in the right lane at the entry to the merge regions.

In order to capture differences in dynamics of passenger cars, heavy trucks and ACC-equipped trucks, different car-following models are used by Zhang et al. (2006). Trucks respond slowly to how the lead passenger cars change speed or location and therefore, vehicles following trucks are presented with a smoother car-following model. Simulation scenarios are carried out based on the lead 
passenger vehicle's acceleration, the follower trucks reaction time and speed limits for trucks.

Cooperative Adaptive Cruise Control (CACC) is a further development of ACC. Van Arem et al. (2006) has studied the impacts of the system on traffic flow characteristics. The stochastic simulation model MIXIC developed by Van Arem et al. (1997) has been used in order to assess impacts of CACC, since the traffic simulation model was considered suitable to study different assumptions for functionality of the system, driving behaviour, roadside systems and vehicle dynamics. The functionality of CACC has been designed as a complement to longitudinal sub-models in the traffic simulation model. Since the CACC system takes over a part of the longitudinal movement, a reference acceleration of CACC has been implemented to determine acceleration when the system controls vehicles in the simulation.

Liu et al. (2004) has studied traffic related impacts of Intelligent Speed Adaption (ISA) by traffic simulation modelling. The functionality of ISA has been implemented in the traffic simulation model and the impacts of ISA on traffic congestion, speed distribution and environment has been studied. The results show that ISA is more useful and effective in less dense traffic. However, ISA can have a positive effect on traffic safety due to reduction in speed variations. Modelling changes in driving behaviour due to ISA, is mentioned as a further step in this research.

Hegeman et al. (2009) has investigated the potential effects of an overtaking assistant system for two-lane rural roads, in a microsimulation study. Most traffic simulation based investigations of ADAS have mainly focused on the longitudinal control of vehicles. It is notable that Hegeman et al. (2009) has used traffic simulation modelling for analysing ISA's effects on traffic. These systems support the driver in deciding if an overtaking is safe based on estimation of distance to the next oncoming vehicle. Outcome from a driving simulator study of overtaking systems and changes in drivers' willingness have been used to capture the changes in driving behaviour with respect to this system. Results indicated that the overtaking assistant can be beneficial for traffic safety due to reduction of average time-to-collision. 
Many traffic analysis tools and methodologies have been developed to assist the transport planner and researcher in improving traffic conditions. Optimizing the operations of facilities, simulating and estimation of probable results for proposed traffic design or plan, are the analytical tasks in the traffic that entail various context in these tools. Due to the already complex structure of a traffic simulation model with applications that manage the network design, traffic detectors and controls, vehicle guidance, vehicle tracking, public transport, etc. the system can give a limited estimation of driving behaviour or vehicle conditions, for example. Vehicle dynamics are often simplified in such simulations. The car-following model normally considers a maximum possible acceleration, but the maximum acceleration does not always depend on the current speed, slope, roll and air resistance, etc.

The current trend in the development of ADAS and increased complexity of these systems, sometimes require integration of two or more tools and methods for evaluation. This is due to the fact that it is difficult to model the detailed functionality of the ADAS in a traffic simulation software, and capture various driving behaviour, vehicle condition and traffic situations at the same time. Different set ups of tools and methods have been examined in several ways. Golias et al. (2002) have presented a methodology for estimation of ADAS impacts that propose a combination of a macroscopic and a microscopic traffic simulation and an emission model. The outcome from microscopic simulation, such as the mean speed of the simulated traffic and headway information, uses as input parameters in the macroscopic simulation and emission model.

Another approach was used by Pereira et al. (2012) when combining a robotics simulator including no detailed simulation of the powertrain and vehicle dynamics. A rather realistic traffic flow would be possible to create, with the integration of these two types of simulators. The robotic simulator would allow the practitioners to consider the robotic nature of an autonomous vehicle and the statistical tools available in the traffic simulator help them to understand the advantages of having this kind of technology in real traffic.

van Noort et al. (2011) have tried to find a link between an experiment and the societal effect in cases of linking micro- and macrosimulation. Microsimulation models consider individual vehicles in relatively small networks. To analyse the benefits on a higher scale a macrosimulation modelling has been used and the 
authors identify two major issues. The input to the macrosimulation model is the fundamental diagrams and not individual vehicle variables. Furthermore, the macrosimulation handles the route choice and it is assumed that the drivers in a network have broad information about traffic conditions.

(Rakha et al. (2004) evaluated vehicle simulation sub-models to enhance vehicle dynamics modelling in traffic simulation models. A major problem with traffic simulation models is that vehicle accelerations in the car-following model do not necessary impose the vehicle dynamics constraints. The real force provided by the engine based on air and rolling resistance force and other roadway grades, are not taken into account. The calibration of the simulation models is difficult, since they do not fit the field data in various cases. In order to determine the vehicles' actual movements, a number of acceleration models based on vehicle dynamics were developed. This method is more flexible than a traffic simulation model and results show a good fit to field data.

Another example is the combining of traffic and driving simulators for enhanced simulation of the surrounding vehicles. (Punzo 2011) state that the integration of traffic and driving simulation may provide a more reliable evaluation result. The behaviour of surrounding vehicles has to be taken into account, in order to accomplish realistic simulations. In a driving simulator, it is possible only if autonomous vehicles in the driving scenario drive according to the autonomous system during the simulation. Indeed, this is not the case in real traffic.

In fact, many researchers state that there is a lack of results' reliability due to limitation in evaluation methods. Numerous studies have been conducted in order to find combinations of methods for evaluating ADAS impacts. Henclewood et al. (2009) state that, "existing vehicle dynamic models, owing to their complexity for high modelling fidelity, are not suited for efficient traffic simulation involving many vehicles". One general limitation for traffic simulation models is that they are not able to represent the newly developed ADAS. For example, microscopic simulation models are not suitable to model the driving behaviour of the interacting and communicating vehicles. 
Chapter 3. ADAS evaluation 


\section{Chapter 4}

\section{Simulation based evaluation framework}

This chapter presents a simulation based evaluation framework for ADAS, an evaluation framework that includes modules that handle vehicle dynamics and ADAS functionality, driver behaviour and the different traffic situations. The following aspects are considered:

- Vehicle: vehicle dynamics and ADAS functionality.

- Driver: drivers' acceptance level and changes in driving behaviour when using ADAS.

- Traffic system: interactions between ADAS equipped and non-equipped vehicles.

The aim of this evaluation framework is to address ADAS impacts on the traffic system at an aggregated level. An ADAS might be developed to reduce air pollution, increase road safety, level-of-service, or traffic throughput. Gains and 
losses may vary for ADAS equipped and non-equipped vehicles as well as for different types of vehicles. Investigations of the real benefits of an ADAS therefore need to include impacts on the traffic system, and not only on vehicle dynamics and driving behaviour.

The need of additional methods for traffic simulation models for ADAS evaluation is reviewed in Section 3.3. This chapter describes the idea of combining a traffic and a vehicle simulation model, and the implementation and verification of the combined model.

\subsection{Combining simulation methods}

The approach developed here represents a further development of the combined traffic and vehicle simulation presented by Tapani (2012). The commercial traffic simulation software, Vissim (Fellendorf et al. 2010), was combined with a vehicle simulation model from Volvo's proprietary global simulation platform (GSP). These simulation models were combined to compare electric and conventional diesel vehicles for city delivery operations. The traffic simulation model provided the vehicle simulation with both a realistic description of urban traffic conditions and an urban truck driver model. The vehicle simulation concerned longitudinal movement of the vehicle and the traffic simulation model determined lateral movement such as lane changing and overtaking. The vehicle simulation determined chassis and driveline dynamics based on information about the vehicles load, weight and position on the road and the roads vertical grade. This integrated simulation approach is considered to be able to yield a detailed analysis of vehicle properties in various traffic conditions.

Two main advantages are expected from combining theory and models from traffic and vehicle simulation fields. First, traffic simulation can be used to support vehicle simulation by supplying a more realistic driver model and more realistic traffic surroundings. Second, vehicle simulation can be used to enhance the modelling of traffic-simulated vehicle dynamics. Vehicle simulation entails simulating a single vehicle's dynamics, powertrain, control algorithms, ADAS and other support systems, etc. This approach improves the validity of traffic simulation results since simplification of ADAS functionality is avoided. 
Furthermore, the car-following model normally considers a maximum possible acceleration, but this maximum acceleration does not always take the current speed, slope, roll and air resistance, etc. into account in the calculations. This is a problem when simulating heavy vehicles travelling on roads with varying slopes.

Another advantage of the approach is that the ADAS implementation can be confidentially retained, allowing traffic simulation analysis of various ADAS designs even in early stages of ADAS development. As a bonus, the concept also results in more detailed modelling of simulated vehicle dynamics compared with the standard approach in traffic simulation models.

Figure 4.1 illustrates the proposed evaluation framework structure in this thesis based on a combination of a vehicle simulation, a traffic simulation and a driving behaviour model to deal with the drivers' interaction with the ADAS. To be able to capture changes in vehicle dynamics, a vehicle simulation model is used as a sub-module of the framework. Changes in driving behaviour in different traffic situations are modelled in a driver model implemented in the traffic simulation model's API. The traffic simulation model determines the traffic conditions and vehicle interactions in the traffic.

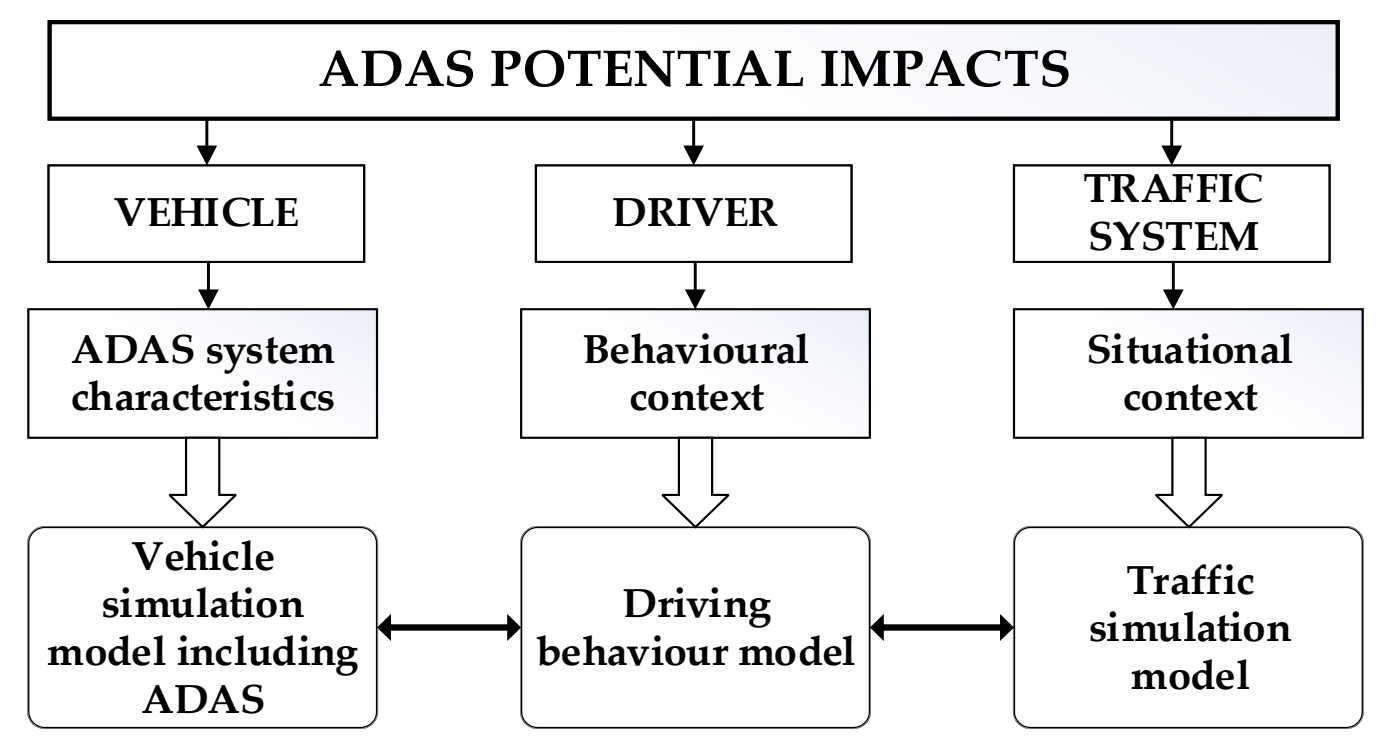

Figure 4.1. Evaluation framework including sub-models 


\subsection{Framework structure}

The framework developed consists of three main components:

- A traffic simulation model

- An external module including the vehicle and driver support system simulation model and a driver sub-model.

- An Application Programming Interface (API) used to connect the traffic simulation model and the external module

The traffic simulation model is the foundation of the framework. Most traffic simulation models provide APIs that can be used to customize the user's simulation models with own applications. For example, the API provides the option to replace or combine existing driver models by a user-defined driving behaviour model for some or all vehicles in a simulation. The basic principle is that the traffic simulation model updates the vehicles behaviour according to the standard behaviour models but that some parts of the behaviour can be overridden via the API. The API also offers the possibility to connect an external vehicle simulation model that includes the "real vehicle" ADAS implementation. In the framework, an external module including both a vehicle and driver support simulation as well as an additional driver sub behaviour model, is connected to the traffic simulation using the API. The following sections will describe the traffic simulation part, the external module and the API.

\subsubsection{The traffic simulation}

The traffic simulation model updates the vehicles desired acceleration, lane, and speed, etc. using the underlying sub behavioural models for car-following, lanechanging, etc. There are several traffic simulation models with accompanying APIs that could be utilized. For the traffic simulation part of the framework, both the Aimsun (v. 7.0) (Casas et al. 2010) micro simulator using the Aimsun API (TSS-Transport 2011) and Vissim (v. 5.4) (Fellendorf et al. 2010) using the Vissim Driver Model API (Hengst 2010) were tested. The Aimsun API allows direct control of vehicle speed while the Vissim Driver Model API only allows acceleration to be overridden. This is probably because Vissim uses a version of the Wiedemann car-following model Wiedemann (1974), which controls vehicle 
acceleration, while Aimsun uses a version of the Gipps car-following model, Gipps (1981), which specifies a vehicle's longitudinal movement from its speed.

\subsubsection{The external module}

A vehicle simulation model developed by truck manufacturer Scania was used for the vehicle simulation part of the external module in the framework. The vehicle simulation model includes modelling of the following:

- Powertrain (engine, gearbox, etc.)

- Vehicle dynamics (roll and air resistance, speed as a function of engine, engine speed, road slope, gear, driving resistance, etc.)

- Driver support systems (different types of cruise control such as CC, LACC and downhill speed control (DHSC))

The implemented driver-support system was taken from that used in real trucks, with some minor modifications. Standard models were used for the powertrain and vehicle dynamics parts. Vehicle simulation was delimited to the longitudinal dimension and did not model the lateral dimension. Vehicle simulation corresponded to a Scania long-haul truck with a $440 \mathrm{hp}(\sim 328.24 \mathrm{~kW})$ engine. In the simulations the weight was set to 38 tons.

The external driver sub model is based on two main parts: the determination of de/reactivating cruise control and calculation of desired acceleration in each simulation step. The development of the driver sub-model and the model itself is presented in detail in Chapter 5.

\subsubsection{The API}

This section describes the details on the interconnection of the microscopic traffic simulation model and external module, including the vehicle and driver support system simulation model and driver sub model. Except for initializing the simulations and functionality for saving vehicle trajectories to file, communication via the API-module consists of two main parts: 
- Creating a new vehicle

- Updating existing vehicles in each simulation step

\section{Creating a new vehicle}

When adding a new vehicle to the traffic simulation, the API implementation checks whether or not the vehicle should be assigned to an external vehicle simulation model. If so, a new instance of the vehicle simulation model is created and initialized. In our case all trucks are assigned an external vehicle model while cars are not. Trucks are also assigned either a regular cruise controller (CC) or a Look Ahead Cruise Controller (LACC).

The vehicle simulation typically assumes that the vehicle starts from a speed of zero while vehicles added to the traffic simulation do not; therefore, the vehicle simulation model is initialized to a steady start-up state.

\section{Updating existing vehicles}

In each time step, vehicles currently available in the simulated road network are updated first in the traffic simulation model, and then in the external module. Vehicles not assigned an external vehicle simulation model are only logged. For vehicles assigned an external vehicle model, two types of updates are required. First, an additional behaviour update is conducted taking into account changes in driver behaviour. This can involve changes in lane changing or car following behaviour, changing the cruise control set speed, or de/reactivation of the cruise control, and is further described in Chapter 5. Second, the vehicle's dynamics are updated using its individual instance of the vehicle simulation model. Vehicle simulation update can be separated into two parts: first, updating the current state (e.g. position, desired acceleration or cruise control set speed) of the vehicle in the vehicle simulation model and, second, running the vehicle simulation model taking into account differences in update frequency. Vehicle simulation models commonly use a higher update frequency than do traffic simulation models, so the vehicle simulation model may have to be run for several iterations in one time step of the traffic simulation model. In our case, traffic simulation was updated at $10 \mathrm{~Hz}$ while $100 \mathrm{~Hz}$ was used for vehicle simulation. 


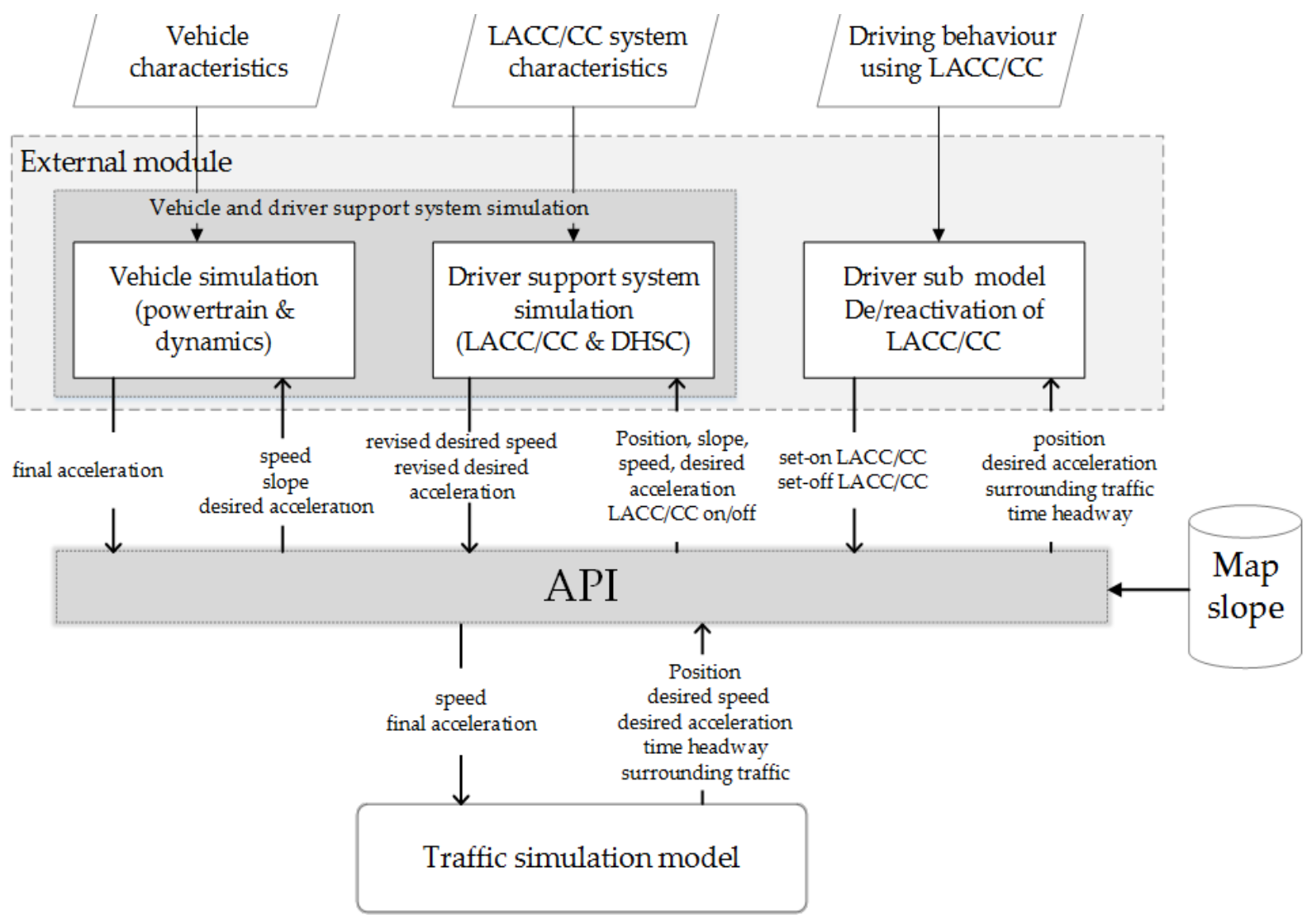

Figure 4.2 Data flow and schematic of the interconnection between the traffic simulation model and vehicle simulation model.

Figure 4.2 also includes the data flows between the microscopic traffic simulation model and external module as well as between the different parts of the external module. The update process can be divided into five main steps:

1. In each time step of the simulation, the traffic simulation updates the vehicles' position and its behaviour including calculating an acceleration that the simulated driver would like to adapt, desired acceleration. The vehicle's position and desired acceleration together with information about the surrounding vehicles are sent to the external driver sub model for de- and reactivation of LACC/CC. 
2. The driver sub model estimates whether the driver will de- or reactivate the LACC/CC. The output of the driver sub model is either to reactivate/keep active or deactivate/keep deactivated.

3. When LACC/CC is activated the driver support system simulation submodule calculates a reference speed, revised desired speed, which also implies a revised desired acceleration. If LACC/CC is not activated the revised desired acceleration will be set equal to the desired acceleration (calculated in the behavioural update in the traffic simulation model)

4. The vehicle simulation then estimates the actual possible acceleration based on the vehicles current speed, the road slope, and engine power. If the revised desired acceleration is higher than possible acceleration, final acceleration is limited to the actual possible acceleration. The final acceleration is then used to update the vehicles speed.

5. The final acceleration and speed is fed back to the traffic simulation and is used in the following time step to update the vehicle's position.

Since some APIs, e.g. the Vissim API, only allows control of acceleration, a vehicle's speed calculated in the traffic simulation update may deviate from its speed calculated in the vehicle simulation update. Tapani et al. (2012) resolved this divergence by adding a correction factor, proportional to the difference in vehicle speed between the traffic and vehicle simulations, to the acceleration feedback in Vissim. This approach, which minimizes any potential deviation over time, has also been adopted and applied in this framework.

\subsection{Verification}

In order to verify that the interconnection works as intended, a verification test using both Aimsun and Vissim was conducted. Two different ADAS's were used in the framework verification. The first one is a regular truck cruise control (CC). Cruise controls for trucks are usually separated into one cruise control to maintain the speed and one downhill speed control (DHSC) that uses the auxiliary brake systems to maintain a desired downhill (set) speed (for a more detailed description, see Section 2.3). In the simulations, the set speed of the 
DHSC was set $5 \mathrm{~km} / \mathrm{h}$ higher than the set speed of the CC (which in the verification runs was set to $85 \mathrm{~km} / \mathrm{h}$ ). The second ADAS is the Look Ahead Cruise Control (LACC) for trucks and heavy vehicles, developed by Scania (2011). This cruise control is a further development of the work of Hellström (2010). The aim of LACC is to minimize fuel consumption based on on-board look-ahead road topography information (see section 2.4 for a detailed description of LACC).

\subsubsection{Single-vehicle trajectories}

To confirm that the interconnection works correctly, the outcomes of the vehicle simulation and combined traffic and vehicle simulation were compared. The vehicle simulation took into account three driver-support systems: cruise control (CC) and look-ahead cruise control (LACC). Another ADAS that was included in the vehicle simulation was downhill speed control (DHSC).

Four combinations were considered, in which either LACC or CC is active:

- LACC active and DHSC active

- LACC active and DHSC inactive

- CC active and DHSC active

- CC active and DHSC inactive

LACC adapt the vehicle's speed based on the road slope, along a road with varying topography. The simulation tests represent an $80-\mathrm{km}$ motorway segment between Södertälje and Nyköping in Sweden with varying topography.

Figures 4.4 and 4.5 illustrate the speed of an equipped truck travelling along this road segment simulated using Aimsun and Vissim, respectively. The outcome from the combined traffic and vehicle simulation was compared with the outcome from a separate simulation with the vehicle simulation. The four combinations of cruise controllers described above have been examined and the speed profiles are shown in the figures. There are no significant differences between the outcomes and the curves seems to fit each other. This verifies that data flows in the interconnected simulation model works correctly. 

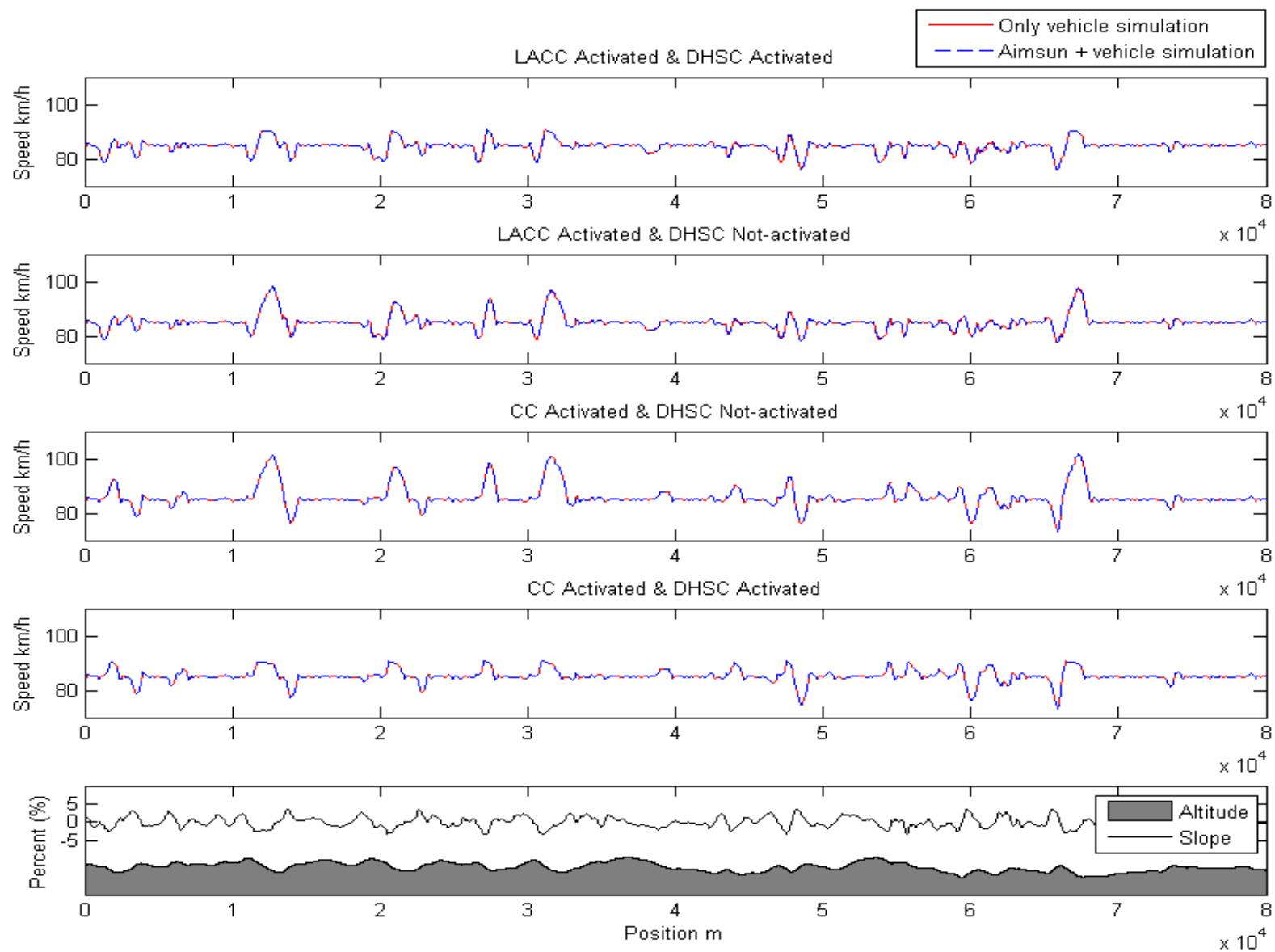

Figure 4.4 Comparison of combined Aimsun plus vehicle simulation and only vehicle simulation for a single truck. 

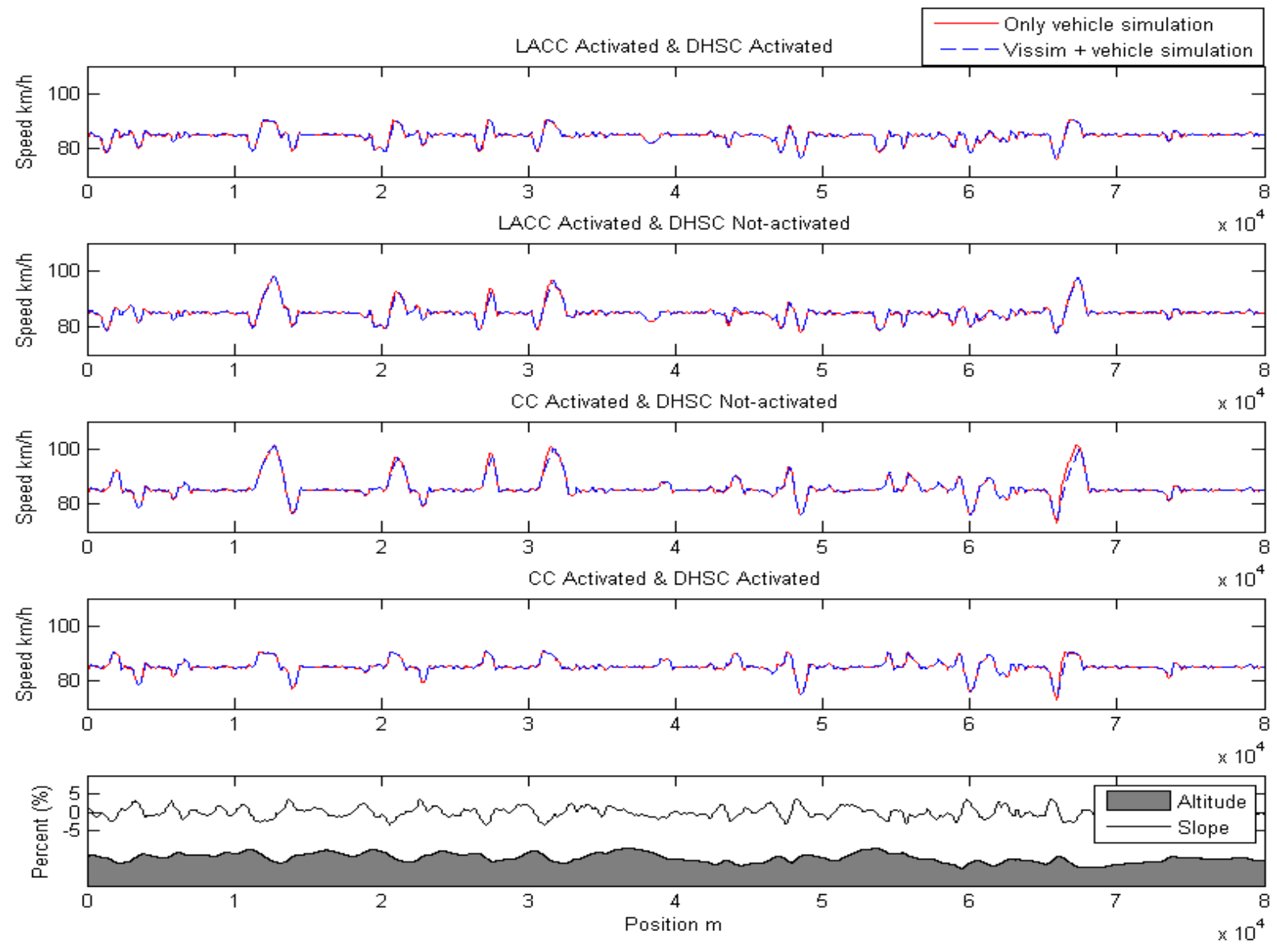

Figure 4.5 Comparison of combined Vissim plus vehicle simulation and only vehicle simulation for a single truck.

Theil's inequality coefficient, Theil (1966), was used as a statistical test to compare the two datasets generated from the interconnected simulation and the separate vehicle simulation. Theil's inequality coefficient is defined as:

$$
U=\frac{\sqrt{\frac{1}{n} \sum_{i=1}^{n}\left(x_{1 i}-x_{2 i}\right)^{2}}}{\sqrt{\frac{1}{n} \sum_{i=1}^{n} x_{1 i}{ }^{2}}+{\sqrt{\frac{1}{n} \sum_{i=1}^{n} x_{2 i}}}^{2}}
$$

where $x_{1 i}$ and is $x_{2 i}$ are the speed observations from the reference vehicle simulation and interconnected simulation, respectively. Theil's inequality coefficient can be decomposed into three proportions: the amplitude $\left(U_{m}\right)$, 
variation $\left(U_{s}\right)$, and covariation $\left(U_{c}\right)$. These proportions of $U$ show different aspects of the error between the two datasets.

The amplitude $\left(U_{m}\right)$, variation $\left(U_{s}\right)$, and covariation $\left(U_{c}\right)$ are defined as:

$$
\begin{aligned}
& U_{m}=\frac{\mathrm{n}\left(\overline{x_{1}}-\overline{x_{2}}\right)^{2}}{\sum_{\mathrm{i}=1}^{\mathrm{n}}\left(\mathrm{x}_{1 \mathrm{i}}-\mathrm{x}_{2 \mathrm{i}}\right)^{2}} \\
& U_{s}=\frac{\mathrm{n}\left(\sigma_{1}-\sigma_{2}\right)^{2}}{\sum_{\mathrm{i}=1}^{\mathrm{n}}\left(\mathrm{x}_{1 \mathrm{i}}-\mathrm{x}_{2 \mathrm{i}}\right)^{\prime}} \\
& U_{c}=\frac{2(1-\mathrm{r}) \mathrm{n} \sigma_{1} \sigma_{2}}{\sum_{\mathrm{i}=1}^{\mathrm{n}}\left(\mathrm{x}_{1 \mathrm{i}}-\mathrm{x}_{2 \mathrm{i}}\right)^{\prime}}
\end{aligned}
$$

where $\bar{x}$ and $\sigma$ are the mean and standard deviation, $r$ is the correlation coefficient, and $n$ is the number of observations (in this case, the number of time steps).

Table 4.2 shows $U, U_{m}, U_{s}$, and $U_{c}$ for the speed in the vehicle simulation and the interconnected simulation, using the Aimsun or Vissim traffic simulation model. Note that the speed from the interconnected simulation closely matches the speed from the vehicle simulation, i.e. $U_{m}$ and $U_{s}$ are close to zero and $U_{c}$ is close to one. Speed observations from the Aimsun based simulations display better fit. The explanation is likely the difference in the possibility of overriding the speed in the traffic simulation model. In the Vissim API, only acceleration can be overridden and the speed error regulator was therefore added, see Section 4.2.3. This seems to influence the precision of the results, but a finer calibration of the speed error correction term might enhance the agreement even more. 
Table 4.2 Theil's Inequality Coefficient for the four simulation cases

\begin{tabular}{|c|c|c|c|c|c|}
\hline & & $\boldsymbol{U}$ & $\boldsymbol{U}_{\boldsymbol{m}}$ & $\boldsymbol{U}_{\boldsymbol{s}}$ & $\boldsymbol{U}_{\boldsymbol{c}}$ \\
\hline \multirow{2}{*}{$\begin{array}{c}\text { LACC + } \\
\text { DHSC }\end{array}$} & AIMSUN & 0.0008 & 0.0000 & 0.0071 & 0.9929 \\
\cline { 2 - 6 } & VISSIM & 0.0032 & 0.0577 & 0.0021 & 0.9402 \\
\hline \multirow{2}{*}{ LACC } & AIMSUN & 0.0013 & 0.0030 & 0.0000 & 0.9970 \\
\cline { 2 - 6 } & VISSIM & 0.0028 & 0.0388 & 0.0484 & 0.9128 \\
\hline \multirow{3}{*}{ CC+ DHSC } & AIMSUN & 0.0002 & 0.0000 & 0.0001 & 1.0000 \\
\cline { 2 - 6 } & VISSIM & 0.0036 & 0.0387 & 0.0007 & 0.9606 \\
\hline \multirow{2}{*}{ CC } & AIMSUN & 0.0002 & 0.0000 & 0.0002 & 0.9998 \\
\cline { 2 - 6 } & VISSIM & 0.0036 & 0.0045 & 0.1397 & 0.8558 \\
\hline
\end{tabular}

Trucks on motorways are commonly driven using cruise control whenever possible. Traffic simulations of trucks on the studied motorway could therefore be expected to mimic the simulation results of the CC plus DHSC case. The ability of the Aimsun and Vissim models to simulate trucks on motorways with varying topography was investigated by running two reference simulations without the interconnection to the vehicle and ADAS simulation (see results in Figure. 4.6). The Aimsun model does not capture the truck's inability to maintain speed on upgrades. This is because the maximum acceleration limitation does not influence the speed as long as the driver drives at the desired speed (see the Gipps car-following model Gipps (1981)). The Vissim model, on the other hand, captures the truck's inability to maintain speed on upgrades but does not capture the characteristic increase in speed on downgrades or the truck driver's use of downhill speed controllers (compare the CC active and DHSC active case in Figure. 4.6). 

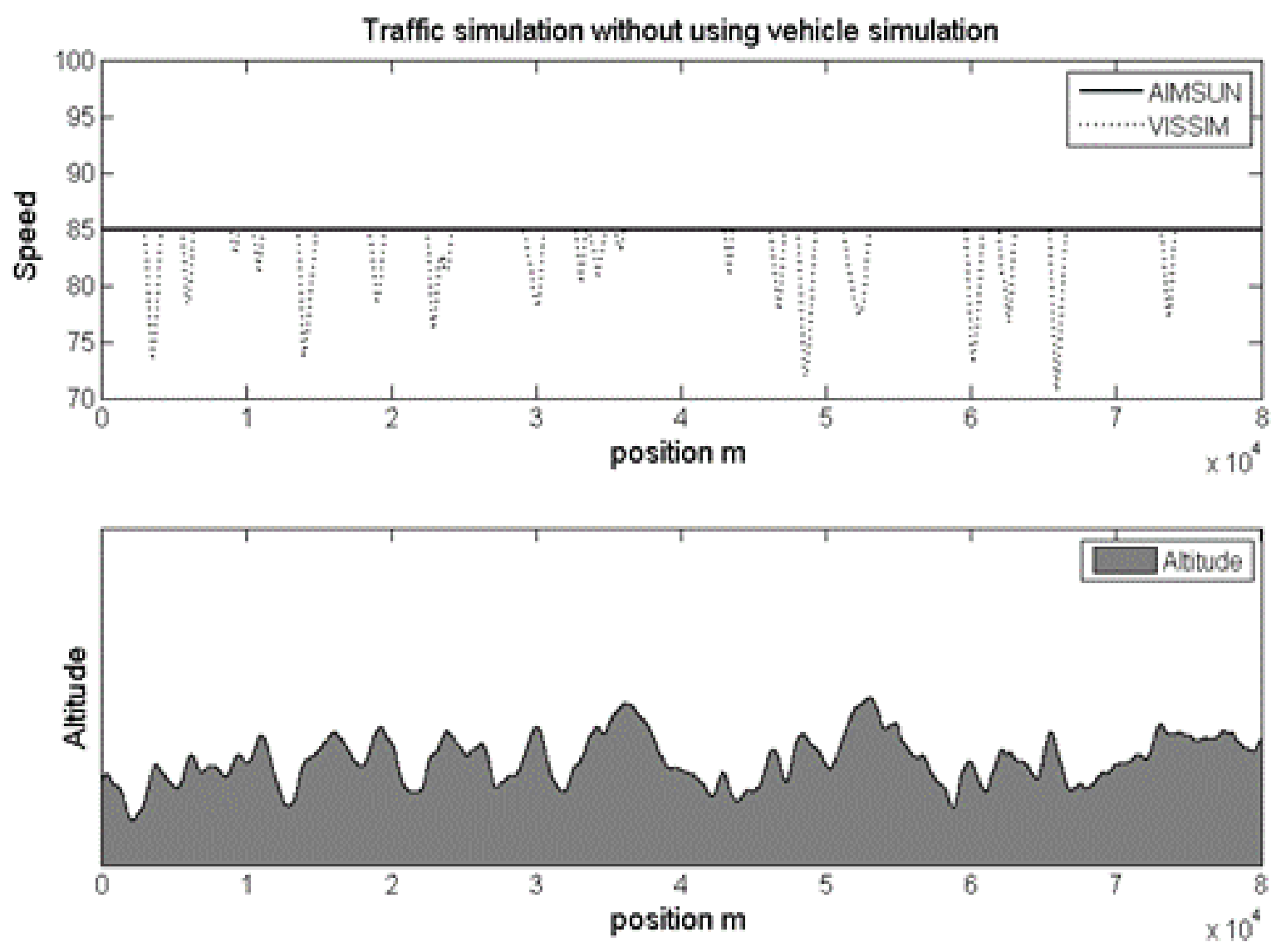

Figure 4.6. Reference simulations without any ADAS or interconnection to the vehicle simulation.

\subsubsection{Simulation of several vehicles}

To ensure applicability of the interconnection approach, simulations of several vehicles were conducted. The aim was to investigate whether sufficiently long road segments, relatively high traffic flow, and high penetration rates of equipped trucks can be simulated within reasonable execution times. The traffic simulation test corresponds to running 400 equipped trucks on the $80-\mathrm{km}$ motorway segment described in section 4.4.1. A two-hour simulation time allowed at least 400 vehicles to traverse the whole road segment, given the desired speed of $85 \mathrm{~km} / \mathrm{h}$. A traffic flow of 400 trucks corresponds to $10 \%$ of the capacity flow for a two-lane motorway (4000 vehicles/h.). All vehicles in the simulation were assumed to be trucks with the same characteristics (e.g. weight, power, and desired speed). The penetration rate was assumed to be $100 \%$. This means that all trucks in the simulation were utilizing LACC for the entire road 
traversal and no deactivation or reactivation driver sub-model was used. The trajectories were saved at $10 \mathrm{~Hz}$ to allow analysis of fuel consumption and driving behaviour.

The run time on a standard laptop computer for the interconnected traffic and vehicle simulation with this set-up is approximately $40-50$ minutes, meaning that the combined model can be run at $2-3$ times real time. This is not extremely fast but workable and acceptable in practice. However, running scenarios with $100 \%$ equipped vehicles on an 80-km road segment at a high flow (e.g. 4000 equipped vehicles/h) might take too much time on a regular desktop computer and would probably require a more powerful computer and/or multithreading.

\subsection{Conclusions}

The interconnection between a traffic and vehicle simulation model presented here, is the first part of the development of a framework for evaluating the effects of powertrain-related ADAS. The first task was to verify the results of the interconnected simulation model, which was done using single-truck simulation tests. Moreover, the framework was applied to a relatively high traffic flow and long road network within reasonable simulation execution times. The presented framework is found suitable for evaluating ADAS that require detailed modelling of the powertrain and/or vehicle dynamics. For other ADAS, the advantage of using the framework might be too small relative to the computational effort.

A secondary conclusion is that the behaviour and vehicle dynamics modelling in Aimsun (v. 7.0) and Vissim (v. 5.4) do not capture the driving characteristics of trucks with cruise control traversing roads with varying topography well enough, at least not to evaluate eco-driving support systems for trucks on motorways.

To be able to model driving behaviour with respect to surrounding vehicles, it is required to have corresponding data available. The Vissim API provides straight forward access to data on closest surrounding vehicles' in each simulation step. The Aimsun API requires an additional program code that in each simulation step searches through the position of all vehicles in the network in order to determine the preceding vehicle or the vehicle in the right lane in an 
Chapter 4. Simulation based evaluation framework

overtaking situation. This is time consuming and requires additional calculations. Therefore it is more practical to use Vissim in the implementation of the framework in this thesis. 


\section{Chapter 5}

\section{Driving behaviour modelling}

In this chapter a driver model is presented. The behavioural driver model simulates the drivers' interaction with regular cruise control (CC) and Look Ahead Cruise Control (LACC). The chapter includes descriptions and driver model structure. The driver model is developed to consider any additional changes in driving behaviour based on drivers' decision for desired or set speed as well as changes in acceleration and deceleration levels, and overtaking behaviour. This driver behaviour model is to be used as a complement to the driving behaviour sub-models in the traffic simulation software and not aimed at replacing any of the available sub-models.

The approach of interconnecting traffic and vehicle simulation model presented in Chapter 4 was the first task in the evaluation of the environmental effects of the LACC. The second task is to observe the LACC's impacts on driving behaviour, both for the equipped vehicle and for surrounding vehicles. The 
identified behavioural effects will then be implemented in a driver model, which will be used to determine when the LACC/CC will be deactivated or reactivated during the simulation. The chapter starts with a description of LACCs effect on truck drivers' behaviour, which is then presented and verified.

\subsection{Observed driving behaviour}

LACC may cause changes in driving behaviour, which in turn can affect the traffic system. In order to consider driving behaviour changes related to LACC, several methodologies have been used in this thesis. We have used a compilation of a Driving Simulator Study, a Field Observation Test (FOT) and a Focus Group Study. The objective in these behavioural studies has been to observe the interaction between the driver and LACC. The methodologies used are described in Section 3.1.

Figure 5.1 shows an overview on how the behavioural studies were planned and conducted. Initial hypotheses were formulated and tested in the Driving Simulator Study and Field Observation Test. The results were then used as input to the design of the Focus Group Study and finally, the findings from all three studies was used in the modelling of de/reactivation of LACC.

\begin{tabular}{|c|c|c|c|c|c|c|}
\hline \multirow{2}{*}{ Initial hypotheses } & results & Driving simulator study & results & \multirow{2}{*}{$\begin{array}{l}\text { Focus Group } \\
\text { Study }\end{array}$} & results & \multirow{2}{*}{$\begin{array}{c}\text { Driver sub-model } \\
\text { de/reactivation of LACC }\end{array}$} \\
\hline & analysis & $\begin{array}{c}\& \\
\text { Field Observation Test }\end{array}$ & analys & & analy & \\
\hline
\end{tabular}

Figure 5.1. Illustration of how outcome from the behavioural studies is used in driving behaviour modelling

\subsubsection{Driving Simulator Study and Field Observation Test}

The driver behavioural study is based on several studies. One is presented by Henriksson P (2012) including the results and analysis of the simulator study and Field Observation Test. The study was conducted by VTI, Swedish National Road and Transport Research Institute and the SCANIA Transportlaboratorium. 24 truck drivers of different age and driving experience participated in the studies. The aim of the Driving simulator experiment was to evaluate LACC at an individual level, for example to investigate how LACC 
influence drivers' willingness and ability to drive in a less energy consuming way. Since CC and LACC have primarily been designed to operate on motorways the scenario created in the driving simulator experiment consisted of a two lane motorway road section. The road section was based on a road section of the E4 national motorway between Södertälje and Nyköping. The experiment contained a warm-up driving of 10 minutes and three different settings: manual driving, driving with CC and driving with LACC.

The LACC was integrated in the dashboard in the driving simulator cabin to ensure the same visual feedback to the driver as in a real truck. The driver could update set-speed and access other settings and real time information using a button panel. More than $80 \%$ of the drivers have completed at least one ecodriving training session. The participants were asked to fill in two questionnaires. The first one included questions about their background and expectations of LACC and was answered before the drive. The second questionnaire contained questions about the driving experience with LACC and was answered after the drive.

A number of dependent measures were considered in the experiment. The main questions were how fuel consumption was affected and how the two alternatives of cruise controllers (CC and LACC) were experienced (accepted). Moreover, speed variations, sleepiness, overtaking probabilities, overtaking rate, etc. were observed. The main hypotheses were:

1. Using the LACC system reduces fuel consumption compared to a situation without.

2. Average desired speed will be lower when using the LACC compared to a situation without.

3. Drivers not using LACC will be more willing to overtake a vehicle equipped with LACC than a vehicle without, given that the vehicles ahead have similar desired speeds.

4. Drivers not using LACC will be more willing to overtake LACCequipped trucks, compared to trucks without. 
5. Drivers using LACC will use similar following time headways as drivers not using this system.

6. LACC will be deactivated more often than CC.

The Field Observation Test was conducted by SCANIA Transportlaboratorium, which is a SCANIA own transport company. In order to investigate how the drivers had experienced driving with LACC, two questionnaires were distributed to the drivers at SCANIA Transportlaboratorium. Three Scania trucks equipped with LACC were driven on the motorway between Södertälje in Sweden and Vordingborg in Denmark. The objective of this test was to observe impressions of LACC and if the drivers noticed any improvements compared to CC. Moreover, in-vehicle data-loggers were used to collect driving data such as speed, position, gear level and braking.

Results of the Simulator Study and Field Observation Test have been discussed by Henriksson P (2012). These studies include the evaluation of users' views on LACC. The Simulator Study results clarify that fuel consumption and average speed decrease when using LACC and the drivers do not deactivate LACC more than CC. The participants show overall positive attitude towards LACC, but they state that they need more experience in driving with LACC to understand if the system realises safer and enhanced fuel-efficient driving. Sleepiness has been difficult to evaluate in these studies.

Participants in these tests state that LACC is more effective in reduction of fuel consumption for inexperienced drivers, which can be true for experienced drivers too, if they are not familiar with the road. The drivers experienced that travel time increased when driving with LACC compared to CC, since the average speed decreased. The lower fuel consumption in LACC-equipped vehicles seems to be affected by both lower average speed and the system function.

In summary, the results of the experiments approve hypotheses 1, 2, 4 and 5 . Hypothesis 3 could not be inferred and hypothesis 6 is rejected. 


\subsubsection{Focus Group Study}

To be able to model driving behavior in the equipped vehicle, it was important to discuss the driving behavior with drivers who have experience in driving trucks equipped with LACC in real traffic. We therefor designed a Focus Group Study in order to give input to modelling driving behavior in a simulation based evaluation framework, see Appendix II. An advantage of the Focus Group Study was to afford the drivers an opportunity to discuss the advantages and disadvantages of LACC and to express how they react in different traffic situations. More specifically, it was essential to study when the drivers decided to deactivate or reactivate the cruise control. Several questions were formulated based on different traffic situations.

The ambition was to meet several drivers at the same time and arrange focus groups. We decided to meet the drivers at the change-station in Ishøj. Another way to meet the drivers is to arrange a meeting with each driver. This would be more time consuming than having focus groups. Another advantage of discussions in focus groups is to have the possibility to discuss the topics with other drivers. Three focus groups with 6,5 and 4 participants were formed based on their arrival time to the station. These participants were not selected based on any qualifications, age or experience. The only selection criteria was having some experience in driving with LACC, they did not receive any information or outline of the study beforehand.

The Focus Group Study was based on qualitative data analysis revealed from discussions in the three focus groups. The purpose of the Focus Group Study was to study interaction between the driver and the LACC with respect to system acceptance and travel time impacts. The Focus Group Study experiment was led by two discussion leaders from VTI who guided the discussions, one of them is the author of this thesis and the other is a more experienced behavior researcher. Discussions were recorded digitally and answers and comments were summarized. The experiment was conducted in June 2013.

The drivers distribute goods between SCANIAS factories. A usual route for these drivers was a round-trip between Södertälje and Vordingborg. The driving scheme was divided in two parts. There was a change-station in Ishøj in Denmark. A drive might be Södertälje-Ishøj or Ishøj-Vordingborg with a few 
hours of rest in Ishøj. The discussion leaders were in Ishøj when the drivers arrived at the station.

The accomplishment of a driver support system depends partly on the drivers' acceptance and willingness to interact with the system, and partly on the interaction during the driving. It is therefor important to study both acceptance of the system and driving behaviour. Acceptance of LACC has been studied with respect to the time that the drivers decide to have the system activated, and to the set-speed the drivers choose. For example if the drivers decide to adjust the set-speed to avoid increased travel time. Moreover, the drivers' decisions were discussed in traffic situations when

1) the vehicle in front is slower.

2) there is a risk for collision.

3) the traffic is dense.

4) the vehicle is in an overtaking situation.

5) the vehicle is going to merge from an on-ramp.

6) the vehicle is going uphill or downhill.

The main findings of the focus group study can be summarized as:

- Many participants state that they do not deactivate LACC when the vehicle is constrained by a slower vehicle ahead. They estimate initially if an overtaking is possible. When it is not possible to overtake, they will press the brake pedal to adjust the speed. Many trucks have been equipped with Adaptive Cruise Control (ACC), which adapts the speed according the preceding vehicle if it is slower. Therefore, they do not for example, have to press the brake pedal to decelerate and deactivate the LACC when ACC is activated. However, they will deactivate LACC when they get constrained by a slower vehicle ahead, if the truck is not equipped with ACC. Some of the drivers state that they deactivate the ACC when lane-changing.

- The truck drivers use LACC and CC whenever possible, i.e. when traffic is not too dense and they are not constrained by other vehicles.

- Average speed when using LACC is lower than when using CC. 
- Total travel time for LACC is higher than for CC.

- Changes in total travel time are small and it doesn't influence the set-speed. In other words, the drivers don't set the LACC set-speed at a higher level to compensate lower average speed caused by using LACC.

- The drivers state that they deactivate LACC by accelerating to a slightly higher speed if an overtaking takes too long due to a too small speed difference with the vehicle they are overtaking.

- The drivers state that they usually do not deactivate LACC when merging from an on-ramp.

- The drivers will join a platoon ${ }^{5}$ if the platoon includes trucks from the same company. Generally, it seems to be more difficult to join a platoon when driving with LACC, since it is not easy to adapt the speed after the platoon.

- Downhill, some of the drivers choose to deactivate LACC in order to keep the speed limits.

- When there are several platoons ahead, drivers deactivate LACC to be able to overtake the platoons.

- It is challenging to have LACC activated when platooning. It requires some speed adaptions using the accelerator or brake pedal in order to maintain the speed of the platoons. If the platoon leader has activated LACC, it will reduce speed close to the top of hill. This makes the following vehicle decelerate earlier and then have to accelerate to avoid drastic speed reduction.

It is worth to noting that the participants in these behavioural studies were truck drivers who worked at SCANIA Transportlaboratorium. They distributed goods between SCANIAs factories. The common route for these drivers was a round-trip between Södertälje and Vordingborg. This means that the drivers were familiar with the road and the reoccurring driving scheme. The fact that many of these drivers have participated in some eco-driving training might

\footnotetext{
${ }^{5}$ A platoon is a group of vehicles that preserve a minimum distance between the vehicles during driving. The main advantage of platooning is to decrease air resistance and energy consumption. A secondary advantage of platooning is increasing the road capacity.
} 
have affected their driving behaviour as well. It can be assumed that the participants were not a perfect representative sample of the truck driver population in Sweden. Since the tests revealed that LACC has a positive influence on the participants, it can clarify that LACC can support all drivers in fuel saving. However, this requires that the drivers have a positive attitude towards the system and are willing to use it whenever possible. Otherwise, the drivers who are unfamiliar with eco-driving may not accept LACC and reject using the system.

\subsubsection{Conclusions}

The conclusions from these behavioural studies have been utilized to model traffic situations when the driver deactivates or reactivates the LACC. The outcome from the Focus Group Study allows us to omit some of these traffic situations, since they seem not to influence the drivers' decision in deactivating or reactivating the LACC. According to the Focus Group Study, an outlined structure can be observed in situations 1, 2, 3, 4 mentioned in Section 5.1.2.

\subsection{Driver model for de/reactivation of LACC}

The LACC adopts the speed without consideration of the traffic situation. This implies that the LACC might control speed in such a way that the vehicle will collide with a slower preceding vehicle if the driver does not take action. In such situations the driver might either act by changing lanes or by deactivating the LACC, e.g. by decelerating. Drivers might also deactivate the LACC or keep it deactivated if they anticipate that the LACC cannot be kept activated long enough (i.e. if a deactivation will be necessary shortly).

A driver's interaction with LACC will affect the proportion of time that the system is active. This obviously has impacts on fuel consumption, compared with when the LACC is active during the entire trip.

Computational models of driving behaviour are often developed to appropriate the most common performance on a task by a population. However, real drivers' interaction with LACC might depend on age, driving style and experience level both in driving and using the LACC. Modelling individual 
differences in behaviour is difficult. The common way to model differences is to assign individual parameter values based on a statistical distribution describing the variation related to various conditions. Variations in driving behaviour for heavy trucks are considerably less than variations in car driving behaviour. Taking this as an advantage, construction of a basic driver model is possible by observing a number of different traffic situations. Therefore, in the model developed in this thesis an average driver is considered and no attention is paid to variation in drivers' experience, gender, age, etc.

LACC and CC controls the longitudinal movement of the vehicle. Therefore, the de/reactivation driver model is designed to interact mainly with the car following model. The car-following model in the traffic simulation software determines the drivers desired longitudinal movements in terms of acceleration during the simulation. The driver model identifies if the vehicle is equipped with LACC or CC and evaluates the surrounding traffic situation. Based on this information the driver model determines if the driver prefers to keep LACC activated or deactivated or if it should be deactivated or reactivated. In other words, the driver model describes in which traffic situations drivers deactivate or reactivate the ADAS. This means that speed and acceleration will be calculated by the vehicle simulation when the LACC/CC is active, and by the car-following model in the traffic simulation when it is not active.

All types of deactivation such as deceleration, acceleration or button press are treated as the driver decides to deactivate/override cruise control. The developed driver model may override the acceleration and desired speed estimated by the traffic simulation model. Changes in desired speed may depend on increased desired speed in order to avoid too long overtaking time or decrease in desired speed in order to maintain safety distance to the proceeding vehicle.

\subsubsection{Model structure}

Figure 5.2 illustrates the decision model for deactivation and reactivation of the cruise control in the equipped vehicle, in each simulation step. The model is designed based on the following basic principles:

1) The truck drivers use the $\mathrm{LACC} / \mathrm{CC}$ whenever possible. 
2) Deactivation is done when the vehicle is constrained by slower vehicles in front, deactivating and adapting speed.

3) Deactivation is done when overtaking is taking too long time (deactivation and acceleration).

4) The cruise control is reactivated when the vehicle is no longer constrained by a slower vehicle or any ongoing overtaking is finished.

These principles are shown by the corresponding numbers in Figure 5.2.

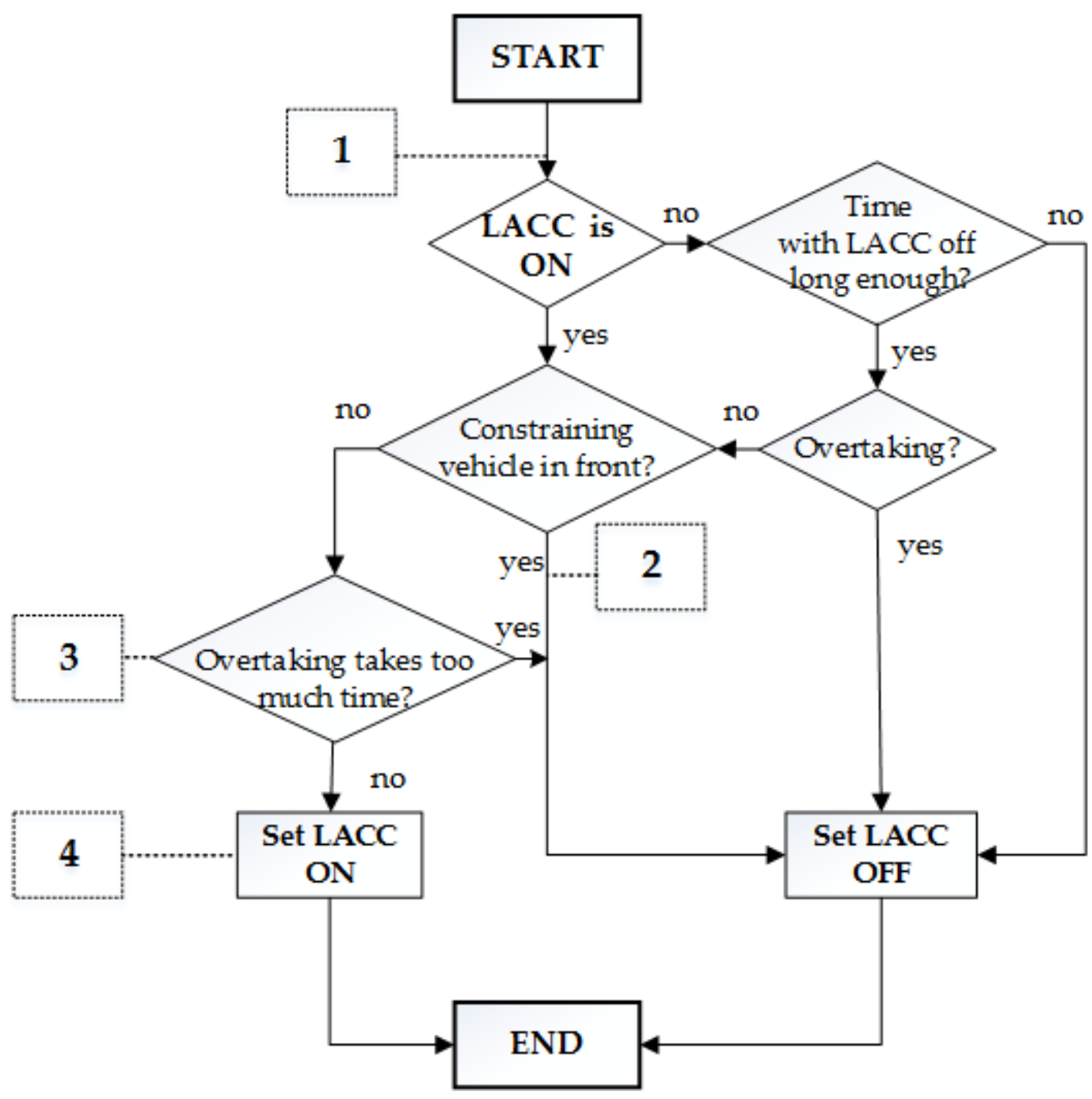

Figure 5.2. Flowchart over the driver model including behavioural update 
It is assumed that the trucks have either CC or LACC and the driver may override cruise control if the traffic situation requires such. The trucks are updated according to the flowchart presented in Figure 5.2. The simulated vehicles' position, speed, acceleration and fuel consumption are updated 10 times per second, which means that every time step in the simulation is 0.1 second.

The behavioural update according to the driver model is made in every time step and involves three main traffic situations:

1. The following situation

2. The overtaking situation

3. Reactivation of LACC/CC

\subsubsection{The following situation}

Consider the traffic situation when a LACC-equipped vehicle is following a slower vehicle. Figure 5.3 illustrates a road section where vehicle $n$ at position $x_{n}(t)$ is catching up vehicle $n-1$ (currently at position $x_{n-1}(t)$ ). It means that the speed $v_{n}(t)$ of vehicle $n$ is higher than the speed $v_{n-1}(t)$ of vehicle $n-1$ and the relative speed between them is positive $\left(v_{n}(t)-v_{n-1}(t)>0\right)$.

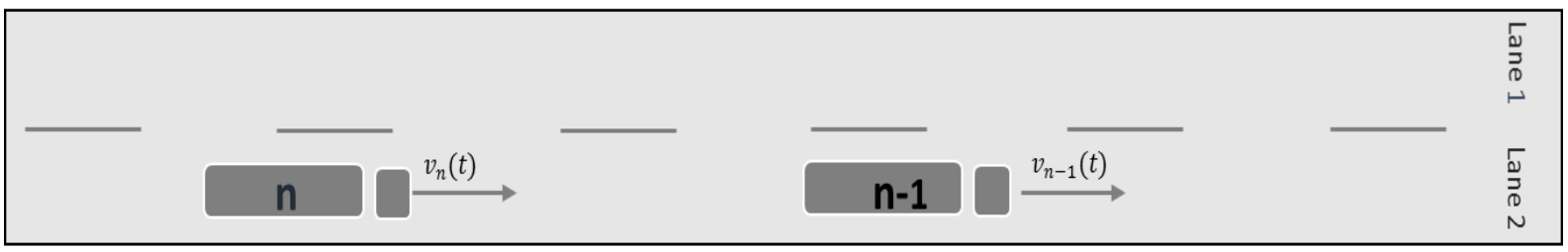

Figure 5.3. Deactivation and reactivation in different traffic situations

If the car-following model suggests a negative acceleration $a_{n}^{C F}(t)$ for vehicle $n$ this is an indication that driver $n$ either drives faster than it's desired speed $v_{n}^{\text {des }}$, e.g. with respect to the speed limit, or need to decelerate in order to avoid a collision with the preceding vehicle $n-1$. If the time headway between vehicles $\left(T H W_{n, n-1}=\frac{x_{n-1}-x_{n}}{v_{n}}\right)$ is small, the reason for deceleration is more probably the leading vehicle, than a speed higher than desired. 
By a combination of information about the suggested acceleration from the carfollowing model, the relative speed and the current headway, it is possible to estimate whether vehicle $n$ is in a following situation or not. In the model developed vehicle $n$ is assumed to be in a following situation when three conditions below are fulfilled:

$$
\begin{aligned}
& v_{n}(t)>v_{n-1}(t) \\
& T H W_{n, n-1}=\frac{x_{n-1}(t)-x_{n}(t)}{v_{n}}<T H W_{\text {min }} \\
& a_{n}^{C F}(t)<0
\end{aligned}
$$

The threshold $T H W_{\min }$ is the longest time headway at which drivers are assumed to take a preceding vehicle into account. In the simulations the $T H W_{\min }$ was set to 6 seconds.

If the LACC/CC is active and the conditions (1)-(3) are fulfilled the driver will deactivate LACC/CC and the acceleration and speed of the vehicle will then be determined by the car-following model. If not all three constraints are fulfilled the driver will continue driving using LACC/CC and acceleration will be determined by the LACC/CC $\left(a_{n}^{L A C C / C C}(t)\right)$. This implies that the final acceleration $a_{n}(t)$ of vehicle $n$ the becomes

$$
a_{n}(t)=\left\{\begin{array}{cc}
a_{n}^{C F}(t) & \text { LACC } / \text { CC deactivated } \\
a_{n}^{L A C C / C C}(t) & \text { LACC } / C \text { activated }
\end{array}\right.
$$

\subsubsection{Overtaking situation}

Consider a traffic situation when a LACC/CC-equipped vehicle is overtaking a slower vehicle. Figure 5.4 illustrates a road section where vehicle $n$ at position $x_{n}(t)$ is overtaking vehicle $n-1$ (currently at position $x_{n-1}(t)$ ). Both the situation where vehicle $n$ is behind vehicle $n-1$ (i.e. $x_{n}(t)<x_{n-1}(t)$ ) and when it is in front of vehicle $n-1$ (i.e. $x_{n}(t)>x_{n-1}(t)$ ) need to be considered. 


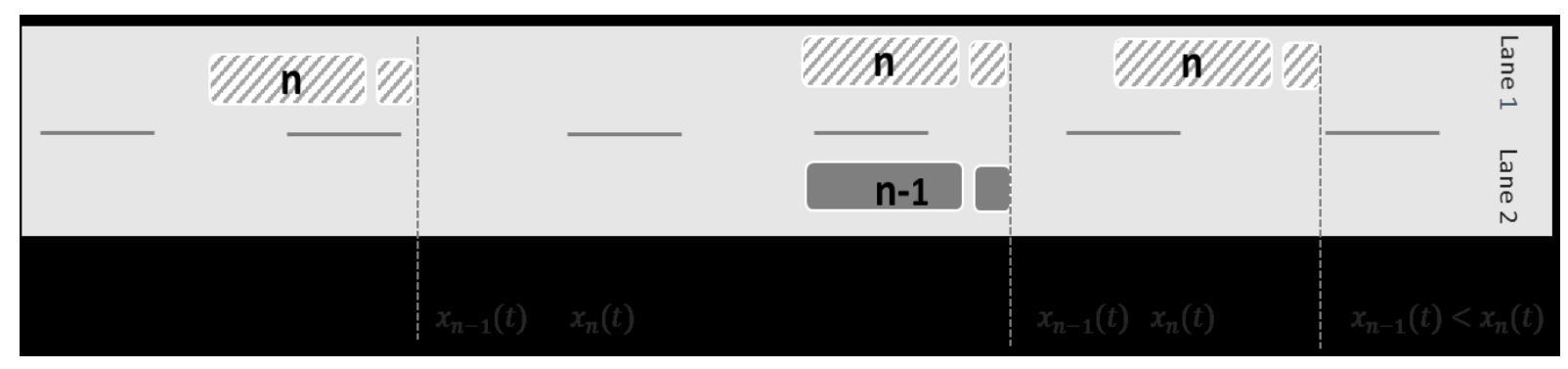

Figure 5.4. The overtaking vehicle is the follower or the leader in different areas during an overtaking situation

LACC-equipped vehicles' speed varies due to the road slope, which may result in that the relative speed $v_{n}(t)-v_{n-1}(t)$ varies (given that vehicle $n-1$ drives at constant speed). If the relative speed becomes less than zero the driver will accelerate in order to avoid that the time needed to finalize the overtaking increases (as stated by drivers in the focus group, see Section 5.1.2). It is assumed that each driver requires a minimum relative speed $\Delta v_{\min }$ to the overtaken vehicle in order to avoid too long overtaking. For example, if vehicle $n$ is currently in the left lane conducting an overtaking, its speed should be at least higher than vehicle $n-1$ in the right lane that is being overtaken. This applies both in the case where vehicle $n$ is behind and in front of vehicle $n-1$. In the driver model LACC/CC is deactivated when:

$$
v_{n}(t)-v_{n-1}(t)<\Delta v_{\min }
$$

When the LACC/CC is deactivated and the vehicle is in the left lane currently overtaking another vehicle $\left(T H W_{n, n-1}<5 s\right)$ the vehicle will increase its desired speed in order to ensure that overtaking will not take too long. The revised desired speed (only used during the overtaking) is calculated as

$$
\tilde{v}_{n}^{\text {des }}(t)=\max \left\{v_{n}^{\text {des }}, v_{n}(t)+\Delta v_{\min }\right\}
$$




\subsubsection{Reactivation of LACC/CC}

If LACC/CC is deactivated the duration for deactivation will be measured. When a driver deactivates LACC/CC due to the traffic situation, it usually takes some time before the driver reactivates the system again. A minimum deactivation time is therefore assumed in order to avoid reactivating the system immediately. However, it is not enough that enough time has elapsed from the deactivation. The driver first checks whether the LACC/CC would have been deactivated if it was active, i.e. checking the conditions for deactivation (1)-(3) and (5) in Section 5.2.1 and 5.2.2.

\subsection{Verification of the driver model}

The simulated vehicles may be passenger cars or trucks. The passenger cars are simulated according to the traffic simulation model and the trucks according to the vehicle simulation model when the cruise control is activated, and by the car-following model in the traffic simulation model, when the LACC/CC is deactivated. When the LACC/CC is deactivated during the simulation, the truck will be updated by the implemented driver model. It is therefore important to verify that the driver model deactivates and reactivates LACC/CC at the correct situations and that the simulation is free from collisions.

Verification of the implemented driver model is performed in order to ensure that:

- The trucks simulated by the driver model behave realistically according to surrounding traffic and that they do not cause collisions.

- The implemented driver model deactivates or reactivates LACC/CC in the correct situations.

- Deactivation and reactivation of the cruise control is modelled correctly in the vehicle simulation model.

- Driving with LACC/CC deactivated gives a correct description of roll and air resistance. 


\subsubsection{Verification of collision free simulations}

The first step in verification of the framework is to confirm if the output data are correctly calculated by the sub-models in the framework. In other words if the interconnection works properly. The detailed description of this step can be found in Section 4.4. The second step is to verify that the driver sub-model does not cause any collisions. A suitable way to verify that the trucks behave realistically and are not involved in any collisions is to analyse the vehicles trajectories. Several simulations have been run and the speed profiles for trucks are observed. An extreme or a quick reduction of speed can indicate a collision. The speed profiles for all trucks in all simulation experiments are observed and they seem to be in an acceptable range.

\subsubsection{Verification of deactivation and reactivation}

A truck should be simulated according to the cruise control simulation whenever possible and it is therefore important to verify if the implemented driver model deactivates the cruise control in the correct situation. Several trucks in different simulation experiments have been considered in two scenarios: 1) where the truck drives freely, without any surrounding traffic and 2) where a truck with the same conditions and desired speed drives in different traffic flows. The comparison between the simulation results show that the generated output from free driving agrees with the output from the simulated traffic with higher traffic flow. In order to verify if deactivation and reactivation of the cruise control is modelled correctly in the vehicle simulation model, several vehicles are selected in various simulation experiments. Figure 5.5 illustrates examples of the verification.

Driving behaviour for trucks is studied with respect to lane changing and relative speed to the preceding vehicle. The desired speed for the studied truck is $82 \mathrm{~km} / \mathrm{h}$. Vissim acceleration is used in the driver sub-model as an indicator to deactivate LACC, because a negative acceleration suggested by Vissim indicates a traffic situation where the truck should decelerate in order to keep a safety time headway, see Section 5.2.1.

Figure 5.5 shows the comparison between the speed profile for a truck equipped with LACC, when the traffic flow is $1700 \mathrm{v} / \mathrm{h}$. The speed profiles for the studied 
trucks feels realistic. For example, at position $x_{1}$ the vehicle deactivates the LACC in a following situation when the vehicle in front is slower and Vissim acceleration is negative. At this point the lane curve shows that the vehicle in traffic has moved from the right lane to the left lane and the vehicle is in an overtaking situation. At position $x_{2}$, the vehicle moves into the right lane after the overtaking and reactivates the LACC. In position $x_{3}$, the vehicle is in the same situation as $x_{1}$ and deactivates the LACC.

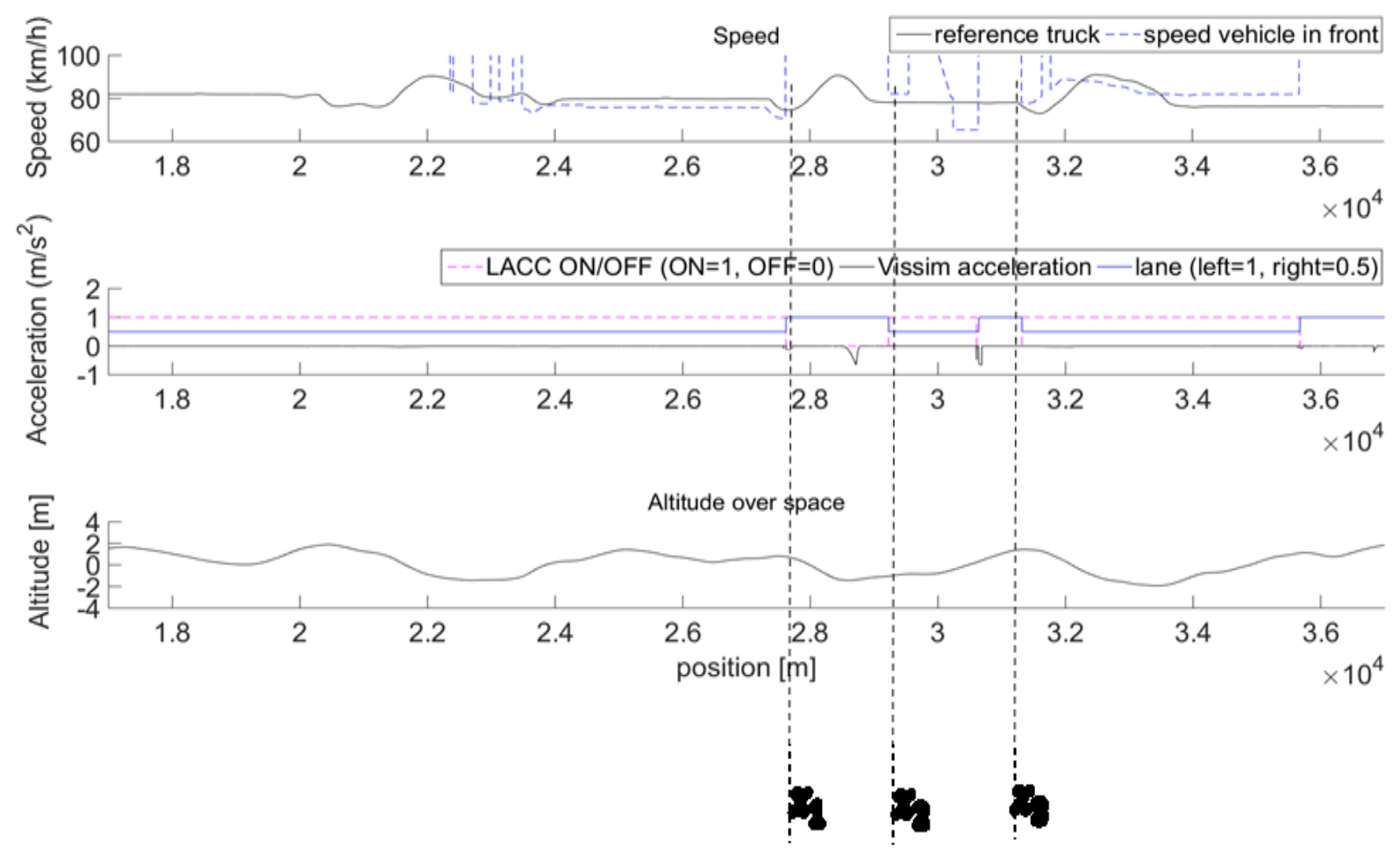

Figure 5.5. Lane-changing and speed profile for a truck and a vehicle in front

\subsubsection{Verification of acceleration}

The Vissim API only allows control of acceleration, the speed of a vehicle in the traffic simulation may deviate from the speed in the vehicle simulation. Tapani et al. (2012) solved this by adding a correction factor, proportional to the difference in speed of the vehicle in the traffic and vehicle simulation, to the acceleration feed back to Vissim. The same approach has been utilized here. 
Both in the driver sub-model and Vissim, speed is calculated based on acceleration by an additional error factor. In each simulation step, acceleration for trucks will be updated by vehicle simulation which has two main parts (in this case). The powertrain and dynamics simulation takes into account engine power, roll resistance, and air resistance, etc. The driver support system simulation estimates acceleration based on the functionality of the cruise controls, LACC, CC and DHSC (for more detailed descriptions see Sections 2.3 and 4.2.1). When LACC/CC is activated, acceleration will be calculated by both parts, and when the cruise control is deactivated, the powertrain and dynamics simulation calculates acceleration. The principal is shown in Figure 5.6. The current acceleration is the vehicles acceleration in the previous time step and the desired acceleration is the acceleration that the car-following model has calculated. The driver sub-model determines if the cruise control will be activated or not and which part of the vehicle simulation will calculate the final acceleration (revised acceleration).

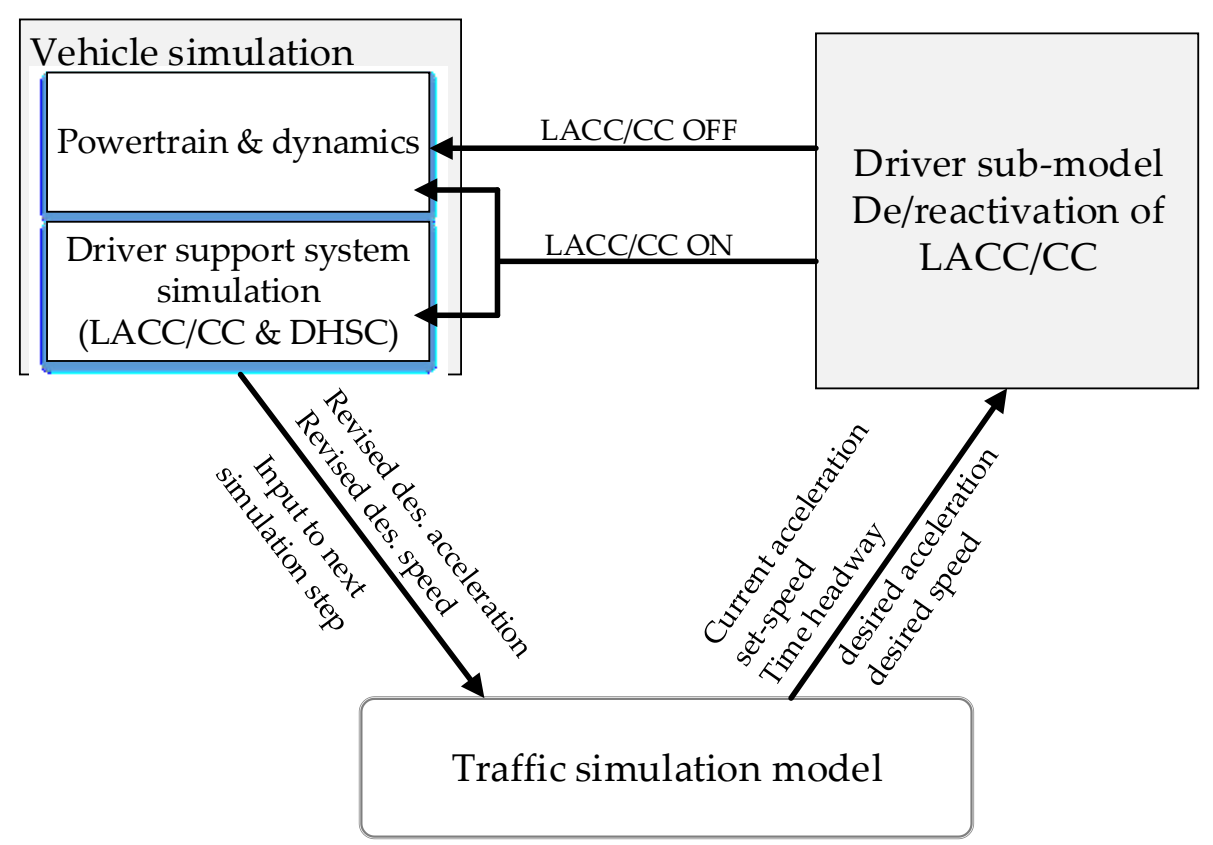

Figure 5.6. The basis of calculation of acceleration in each simulation step. 
Since there are several options for calculating acceleration, it is important to verify if the final acceleration is calculated correctly. Comparison between trajectories for several LACC-equipped trucks shows that the speed calculated by Vissim agrees with the vehicle simulation. The desired speed may have a larger value compared with the current speed the moment deactivation is made. It may take a while to adjust the current speed to desired speed. It can depend on the trucks limited ability to change the speed. The acceleration calculated by Vissim when LACC/CC is deactivated, can be higher than the final acceleration. It depends on recalculation of acceleration by powertrain simulation which takes into account the vehicle condition and roll and air resistance.

\subsection{Conclusions}

The driver sub-model presented in this thesis is able to simulate trucks that are equipped with DHSC and LACC / CC. The model updates the simulated truck based on the vehicle conditions and traffic situation in a realistic way. This external model generates realistic and safe driving behaviour. The input and output data flows correctly in the framework and the calculations of speed and acceleration is completed based on the driver sub-model. 


\section{Chapter 6}

\section{Simulation results}

This chapter describes the experimental design for a range of simulation experiments and discusses the results. The purpose is to investigate the impacts of LACC on the environment and on traffic efficiency, with respect to different penetration rates of LACC in the traffic system. Variations in fuel consumption, travel time and mean speed are studied and presented. Moreover, driving behaviour such as utilization of LACC/CC is observed.

\subsection{Design of traffic simulation experiments}

\subsubsection{Hypothesis}

A traffic simulation experiment was conducted in order to investigate to what extent LACC-equipped vehicles affect other vehicles. Different traffic simulation scenarios were designed to analyse the following hypotheses: 
- LACC will have a positive effect on fuel consumption and on traffic related emissions for LACC-equipped trucks.

- An increased penetration rate of LACC will not have negative effects on emissions for LACC- equipped trucks and CC- equipped trucks.

- An increased penetration rate of LACC will not have any effect on energy use or car emissions.

- LACC will increase travel time for LACC- equipped trucks.

- LACC will not have a negative effect on travel time for cars and CCequipped trucks.

- LACC will have less effect for LACC- equipped trucks in higher traffic flow situations and in denser traffic conditions.

- LACC will have less effect for LACC- equipped trucks and CC- equipped trucks, if drivers' desired speed varies considerably.

The hypotheses were tested with respect to the behaviour when driving with the LACC/CC, given different combinations of LACC penetration level, distribution of desired speed, and traffic flow.

\subsubsection{Performance indicators}

The evaluation was conducted using a set of used performance indicators. In order to study the hypotheses, we have selected several indicators to observe. Table 6.1 presents the indicators, which were selected and determined to examine the hypotheses above. 
Table 6.1 The applied performance indicators for LACC- equipped trucks, CCequipped trucks and cars in the traffic simulation experiments.

\section{Environmental impacts measures}

- Average fuel consumption

- Average emissions of: $\mathrm{CO} 2, \mathrm{CO}, \mathrm{HC}$, and $\mathrm{NOx}$

\section{Traffic efficiency measures}

- Average travel speed

- Average travel time

\section{Utilisation of $\mathrm{LACC} / \mathrm{CC}$}

Average portion of the distance that truck drivers were able to use the cruise control.

The number of LACC-equipped trucks is expected to increase in the future and examination of the effects of this growth is relevant. For the analysis of the traffic performance and environmental effects of LACC usage, differences in the corresponding measures will be analysed with respect to different truck/car flow, and the penetration rates of LACC-trucks. Furthermore, to understand changes in driving behaviour, activation and deactivation of the LACC has been analysed related to the traffic flow, the distance proportion and the proportion of time that the drivers have decided to activate the system.

\subsubsection{Simulation scenarios}

We have designed the simulation scenarios by combining two levels of truck flow and four levels of car flow.

The following combinations were considered (See also Table 6.2):

- Four levels of car traffic flow: 0, 800, 1600 and $2400 \mathrm{v} / \mathrm{h}$.

- Two levels of truck traffic flow: 200 and $400 \mathrm{v} / \mathrm{h}$. 
- Five levels of the proportion of LACC-equipped trucks $0 \%, 25 \%, 50 \%, 75 \%$ and $100 \%$.

The measures presented in Table 6.1 are analysed for LACC-trucks, CC-trucks, cars, and all vehicles in the traffic.

Table 6.2 The scheme for the traffic simulation experiment.

\begin{tabular}{|cc|c|c|}
\hline \multicolumn{3}{|c|}{ Various traffic flow levels (vehicles per hour) } \\
\hline \hline $\begin{array}{c}200 \text { trucks \& } \\
0 \text { cars }\end{array}$ & $\begin{array}{c}200 \text { trucks \& } \\
800 \text { cars }\end{array}$ & $\begin{array}{c}200 \text { trucks \& } \\
1600 \text { cars }\end{array}$ & $\begin{array}{c}200 \text { trucks \& } \\
2400 \text { cars }\end{array}$ \\
\hline \begin{tabular}{c|c|c|c|}
400 trucks \& \\
0 cars
\end{tabular} & $\begin{array}{c}400 \text { trucks \& } \\
800 \text { cars }\end{array}$ & $\begin{array}{c}400 \text { trucks \& } \\
1600 \text { cars }\end{array}$ & $\begin{array}{c}400 \text { trucks \& } \\
2400 \text { cars }\end{array}$ \\
\hline
\end{tabular}

\begin{tabular}{|c|c|c|c|c|}
\hline \multicolumn{5}{|c|}{ Various penetration rates of LACC } \\
\hline $0 \%$ LACC & $25 \%$ LACC & $50 \%$ LACC & $75 \%$ LACC & $100 \%$ LACC \\
\hline
\end{tabular}

\begin{tabular}{|c|c|c|c|}
\hline \multicolumn{4}{|c|}{ Vehicles in the traffic system } \\
\hline TOTAL & LACC-trucks & CC-trucks & CARS \\
\hline
\end{tabular}

\subsubsection{The simulated road}

We have made the simulation experiments for a motorway since this is the type of road where CC are most frequently used. All scenarios in the simulation experiments consist of a straight $40 \mathrm{~km}$ motorway section with two lanes in each direction, a speed limit of $110 \mathrm{~km} / \mathrm{h}$, and a speed limit for the trucks of $80 \mathrm{~km} / \mathrm{h}$. The first $10 \mathrm{~km}$ is used as a warm-up stretch and only the last $30 \mathrm{~km}$ are considered in the calculations of the performance indicators. The simulation time is set to 105 minutes, including 45 minutes warm up. This means that vehicles that exit the simulated motorway during the first 45 minutes are excluded from performance indicators calculations. Calculations of the effects are based on trajectories for the vehicles that exit the motorway after 45 minutes and before 105 minutes. 


\subsubsection{Vehicle characteristics}

Different type of distributions and functions in the traffic simulation model are aimed to deal with the variability of the vehicles' characteristics. Several parameters are assigned based on stochastic distributions to be able to simulate various vehicle types.

The simulation experiments in this study include only cars and trucks. The traffic simulation model determines the characteristics for the simulated cars and the trucks' characteristics are defined by the external module in the combined framework presented in Chapter 4 . All trucks are assumed to be Scania long-haul trucks with a $440 \mathrm{hp}(\sim 328.24 \mathrm{~kW})$ diesel engine and a total weight of 38 tones.

All trucks are assumed to be equipped with CC or LACC to maintain the speed and one downhill speed control (DHSC) that uses the auxiliary brake systems to maintain a desired downhill set-speed.

\subsubsection{Driver characteristics}

The simulated drivers are assumed to set the LACC/CC set speed to their desired speed and the DHSC set speed to a speed $5 \mathrm{~km} / \mathrm{h}$ higher than the set speed of the LACC/CC. A vehicle not constrained by other vehicles will drive at the desired speed.

The desired speed distribution is an important parameter that influences traffic performance and energy use. Strong variations in desired speeds can cause more willingness to overtake. This effect might be more observable when some of the trucks are equipped with LACC, due to the different speed profile for LACC-trucks. Therefore, it is interesting to study LACC/CC impacts with respect to different distribution of the desired speed.

We have also calculated the performance indicators with respect to different desired speed distributions. The minimum and maximum desired speed for trucks are assumed to be $75 \mathrm{~km} / \mathrm{h}$ and $85 \mathrm{~km} / \mathrm{h}$ respectively. The performance indicators presented in Sections 6.2-6.4 are generally calculated based on the simulation results with a uniform distribution for the drivers' desired speed, i.e. the distribution function is a linear function. In this case, the range of the desired 
speed is large. This is the default set up for the desired speed distribution and the results presented in this chapter are based on this kind of desired speed distribution.

The second desired speed distribution used in the simulation experiments, is when the trucks that are assigned desired speeds based on a normal distribution. This means that the desired speed for trucks does not vary as much and the range of the desired speed is smaller.

\subsubsection{Number of replications}

In order to achieve reliable simulation results, a minimum number of repetitions of the simulation model is needed. The number of repetitions is estimated using confidence intervals calculated from a preliminary set of simulations. According to Bång et al. (2014) the required number of repetitions $N$ can be calculated as

$$
N=\left(\frac{s \cdot t_{n-1, \alpha / 2}{ }^{2}}{\bar{m} \cdot \beta}\right)^{2}
$$

where $s$ is the standard deviation for the measures, $t_{n-1, \alpha / 2}$ is the critical value of the t-test for $n-1$ degrees of freedom and significance level $\alpha / 2$. Where $\alpha=$ 0.95 and $\bar{m}$ is the mean value from the preliminary set of simulations. $\beta=5$ is the acceptable error in percent.

$N$ must be estimated by iterative calculations. A preliminary set of repetitions is usually made to estimate the standard deviation of results, which will be used to calculate $N$. For all simulation repetitions studied in this thesis, five repetitions were initially conducted, and the calculation of $N$ showed that less than five repetitions were required for all the relevant performance indicators, and additional repetitions were not necessary. The results presented in the thesis are therefore based on five repetitions of each simulation scenario.

\subsection{Impacts on the environment}

In this section, the environmental impacts are presented and discussed. Fuel consumption and traffic related emissions are measured and analysed. As a 
default choice, the trucks will be assigned desired speed based on a uniform distribution.

\subsubsection{Impacts on fuel consumption}

The mean fuel consumption per $10 \mathrm{~km}$ is calculated for the scenarios described in Table 6.2. In this section, results and analyses are presented based on:

- Fuel consumption for all trucks

- Comparison between LACC- and CC-equipped trucks with uniform distributed desired speeds

- Comparison between LACC- and CC-equipped trucks with uniform vs normal distributed desired speeds

- Comparison between LACC- and CC-equipped trucks with trucks in free-flow traffic

- Fuel consumption for all cars

\section{Fuel consumption for all trucks}

A comparison of the mean fuel consumption for all trucks with respect to different penetration level of LACC-trucks is illustrated in Figure 6.1. Results show that the penetration level of LACC-trucks does not affect fuel consumption in the same way in all scenarios. Fuel consumption seems to decrease with increasing penetration rate for the low traffic flow scenarios. The trend is similar but smaller for the case with $1600 \mathrm{cars} / \mathrm{h}$. The figure shows a growth of fuel consumption for all trucks, when the traffic flow increases. 


\section{Mean fuel all trucks}

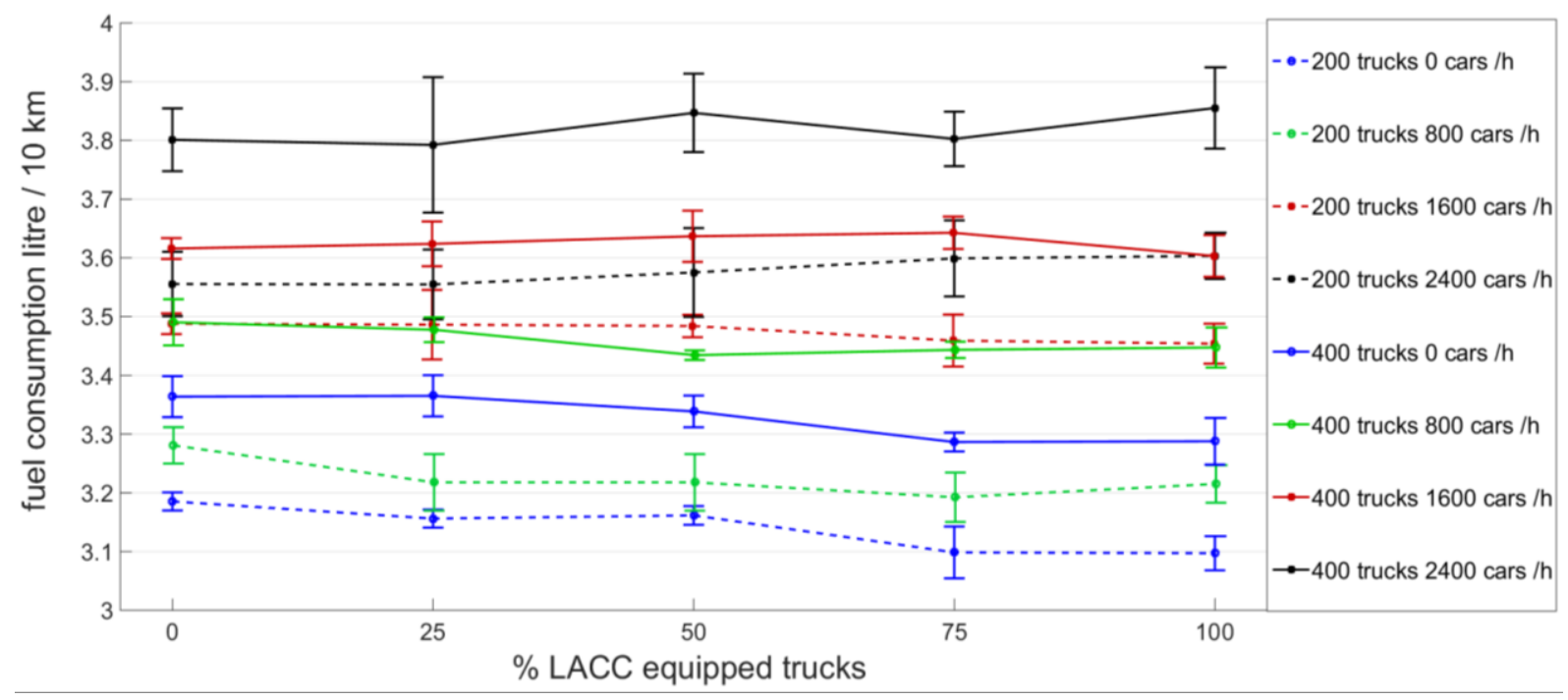

Figure 6.1. Comparison of fuel consumption for all trucks with respect to different penetration level for 8 traffic flows

\section{Comparing LACC- and CC-trucks with uniform distributed desired speed}

The mean fuel consumption for LACC and CC-equipped trucks for different traffic flow conditions is shown in Figure 6.2. The figure shows the fuel consumption for the 8 combinations of traffic flow regarding trucks and cars. The increase in traffic flow is reflected in the results as increased fuel consumption both for LACC- and CC-equipped trucks. An increase in the flow of trucks from 200 to 400 trucks/h influences fuel consumption more than an increase in the flow of passenger cars with $800 \mathrm{cars} / \mathrm{h}$. Although the number of passenger cars increases with 800 , the increase of fuel is less than the scenario with 200 more trucks/h. This indicates, as expected, that the trucks influence the driving behaviour for other trucks. However, we see that differences between the different penetration rates of LACC-equipped trucks are not significant. A comparison between fuel consumption for the LACC and CC-equipped trucks shows that the LACC-equipped trucks have a lower fuel consumption in all scenarios and this difference is more significant when the flow of trucks and cars is low. 


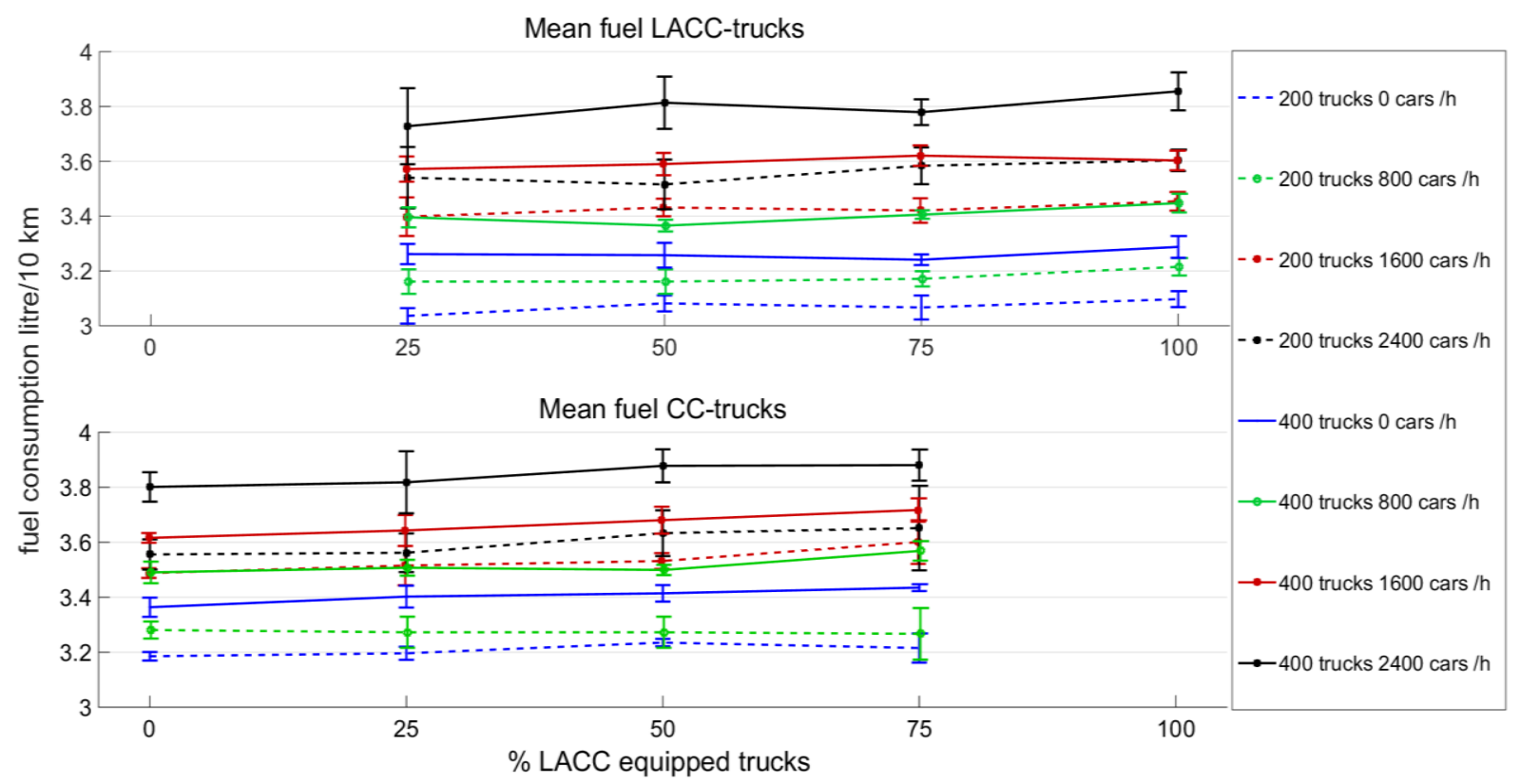

Figure 6.2. Comparison of the fuel consumption for LACC-equipped trucks (top) and CC-equipped trucks (bottom) for 8 traffic flows

Since it is difficult to compare the results for CC and LACC-equipped trucks in the same scenarios in Figure 6.2, we have also presented the results in Tables 6.3 and 6.4. The fuel consumption (litre $/ 10 \mathrm{~km}$ ) for CC-equipped and LACC-trucks is given for four scenarios where the truck flow is $200 \mathrm{v} / \mathrm{h}$ and car flow is 0,800 , 1600 or $2400 \mathrm{v} / \mathrm{h}$. For these scenarios, the improvements in fuel consumption for LACC-equipped trucks compared to the CC-equipped trucks are given in Table 6.4. The results show that LACC is able to decrease fuel consumption in all scenarios. The benefit for an individual vehicle seems to decrease for low car flow. The same trend can be observed when the car flow increases, apart from the scenario where car flow is 1600 or $2400 \mathrm{v} / \mathrm{h}$. It might be that the LACCequipped trucks may constrain CC-equipped trucks when traffic is dense, which causes an increase in fuel consumption for CC-equipped trucks. However, in these scenarios the utilization of the LACC/CC clearly decreases and the results of the fuel consumption may not be relevant to compare with the other scenarios (see more detailed results and analysis in Section 6.2). 
Table 6.3. Comparison of fuel consumption for LACC and CC-equipped trucks when truck flow is $200 \mathrm{v} / \mathrm{h}$

Penetration rates of LACC-trucks

\begin{tabular}{lcccccc}
\hline \multicolumn{1}{c}{ car flow $(\mathrm{v} / \mathbf{h})$} & $\mathbf{0 \%}$ & $\mathbf{2 5 \%}$ & $\mathbf{5 0 \%}$ & $\mathbf{7 5 \%}$ & $\mathbf{1 0 0} \mathbf{0}$ \\
\hline CC-trucks & $\mathbf{0}$ & 3.185 & 3.196 & 3.235 & 3.215 & \\
LACC-trucks & & & 3.036 & 3.082 & 3.067 & 3.097 \\
\hline CC-trucks & $\mathbf{8 0 0}$ & 3.281 & 3.273 & 3.273 & 3.267 & \\
LACC-trucks & & & 3.161 & 3.161 & 3.171 & 3.215 \\
\hline CC-trucks & $\mathbf{1 6 0 0}$ & 3.488 & 3.515 & 3.532 & 3.601 & \\
LACC-trucks & & & 3.398 & 3.431 & 3.421 & 3.454 \\
\hline CC-trucks & $\mathbf{2 4 0 0}$ & 3.556 & 3.562 & 3.633 & 3.652 & \\
LACC-trucks & & & 3.540 & 3.516 & 3.584 & 3.603 \\
\hline
\end{tabular}

Table 6.4. Improvements in fuel consumption for trucks equipped with LACC compared with CC when truck flow is $200 \mathrm{v} / \mathrm{h}$

\begin{tabular}{rrrr}
\hline \multicolumn{4}{c}{ Penetration rates of LACC-trucks } \\
\hline car flow (v/h) & $\mathbf{2 5 \%}$ & $\mathbf{5 0 \%}$ & $\mathbf{7 5 \%}$ \\
$\mathbf{0}$ & $-5.01 \%$ & $-4.74 \%$ & $-4.62 \%$ \\
$\mathbf{8 0 0}$ & $-3.41 \%$ & $-3.41 \%$ & $-2.92 \%$ \\
$\mathbf{1 6 0 0}$ & $-3.33 \%$ & $-2.84 \%$ & $-5.00 \%$ \\
$\mathbf{2 4 0 0}$ & $-0.60 \%$ & $-3.23 \%$ & $-1.86 \%$ \\
\hline
\end{tabular}

The fuel consumption results (litre/10km) for CC- and LACC-equipped trucks when truck flow is $400 \mathrm{v} / \mathrm{h}$, are given in Table 6.5. The four scenarios where the truck flow is $400 \mathrm{v} / \mathrm{h}$ and the flow is $0,800,1600$ or $2400 \mathrm{v} / \mathrm{h}$ are observed. The improvement in fuel consumption for LACC-equipped trucks compared with CC-trucks is given in Table 6.6. The results show the same improvement trend as when the truck flow is $200 \mathrm{v} / \mathrm{h}$.

It was further observed that the fuel consumption decreases more when the penetration level of LACC-equipped trucks increases and the benefits decrease when the flow increases. In the scenario where the car flow is $2400 \mathrm{v} / \mathrm{h}$, we can notice lower improvement when the penetration rate of LACC-trucks is $50 \%$. 
This can indicate that CC-trucks constrain LACC-trucks in dense traffic, which results in more accelerations and decelerations and consequently more fuel consumption.

Table 6.5. Comparison of fuel consumption for LACC- and CC-equipped trucks when truck flow is $400 \mathrm{v} / \mathrm{h}$

Penetration rates of LACC-trucks

\begin{tabular}{lcccccc}
\hline \multicolumn{1}{c}{ car flow $(\mathrm{v} / \mathrm{h})$} & $\mathbf{0 \%}$ & $\mathbf{2 5 \%}$ & $\mathbf{5 0 \%}$ & $\mathbf{7 5 \%}$ & $\mathbf{1 0 0} \%$ \\
\hline CC-trucks & $\mathbf{0}$ & 3.364 & 3.402 & 3.414 & 3.435 & \\
LACC-trucks & & & 3.262 & 3.258 & 3.241 & 3.288 \\
\hline CC-trucks & $\mathbf{8 0 0}$ & 3.490 & 3.507 & 3.499 & 3.569 & \\
LACC-trucks & & & 3.395 & 3.365 & 3.406 & 3.447 \\
\hline CC-trucks & $\mathbf{1 6 0 0}$ & \multirow{2}{*}{3.616} & 3.643 & 3.680 & 3.717 & \\
LACC-trucks & & & 3.572 & 3.590 & 3.621 & 3.603 \\
\hline CC-trucks & $\mathbf{2 4 0 0}$ & 3.801 & 3.818 & 3.878 & 3.881 & \\
LACC-trucks & & & 3.728 & 3.814 & 3.779 & 3.855 \\
\hline
\end{tabular}

Table 6.6. Improvements in fuel consumption for trucks equipped with LACC compared with CC when truck flow is $400 \mathrm{v} / \mathrm{h}$

\begin{tabular}{rrrr}
\hline \multicolumn{4}{c}{ Penetration rates of LACC-trucks } \\
\hline car flow (v/h) & $25 \%$ & $50 \%$ & $75 \%$ \\
0 & $-4.14 \%$ & $-4.59 \%$ & $-5.65 \%$ \\
800 & $-3.19 \%$ & $-3.82 \%$ & $-4.57 \%$ \\
1600 & $-1.96 \%$ & $-2.45 \%$ & $-2.60 \%$ \\
2400 & $-2.35 \%$ & $-1.65 \%$ & $-2.61 \%$ \\
\hline
\end{tabular}




\section{Comparing LACC- and CC-equipped trucks with uniform vs normal distributed desired speeds}

So far in the analysis, we have assumed a uniform distribution of the desired speed for the truck drivers. The mean fuel consumption is further observed when the desired speed of trucks are assigned based on a normal distribution. The mean values of fuel consumption are calculated for the four scenarios based on combining two levels of car flow and two levels of truck flow. The mean fuel consumption for both CC and LACC-equipped trucks seem to increase when the desired speed for trucks are normally distributed, Figure 6.3, compared to the results in Figure 6.2. However, LACC-trucks seem to be influenced more than CC-trucks. A summary of the increase in the mean fuel consumption is presented in Table 6.7 and more detailed tables are available in Appendix II.

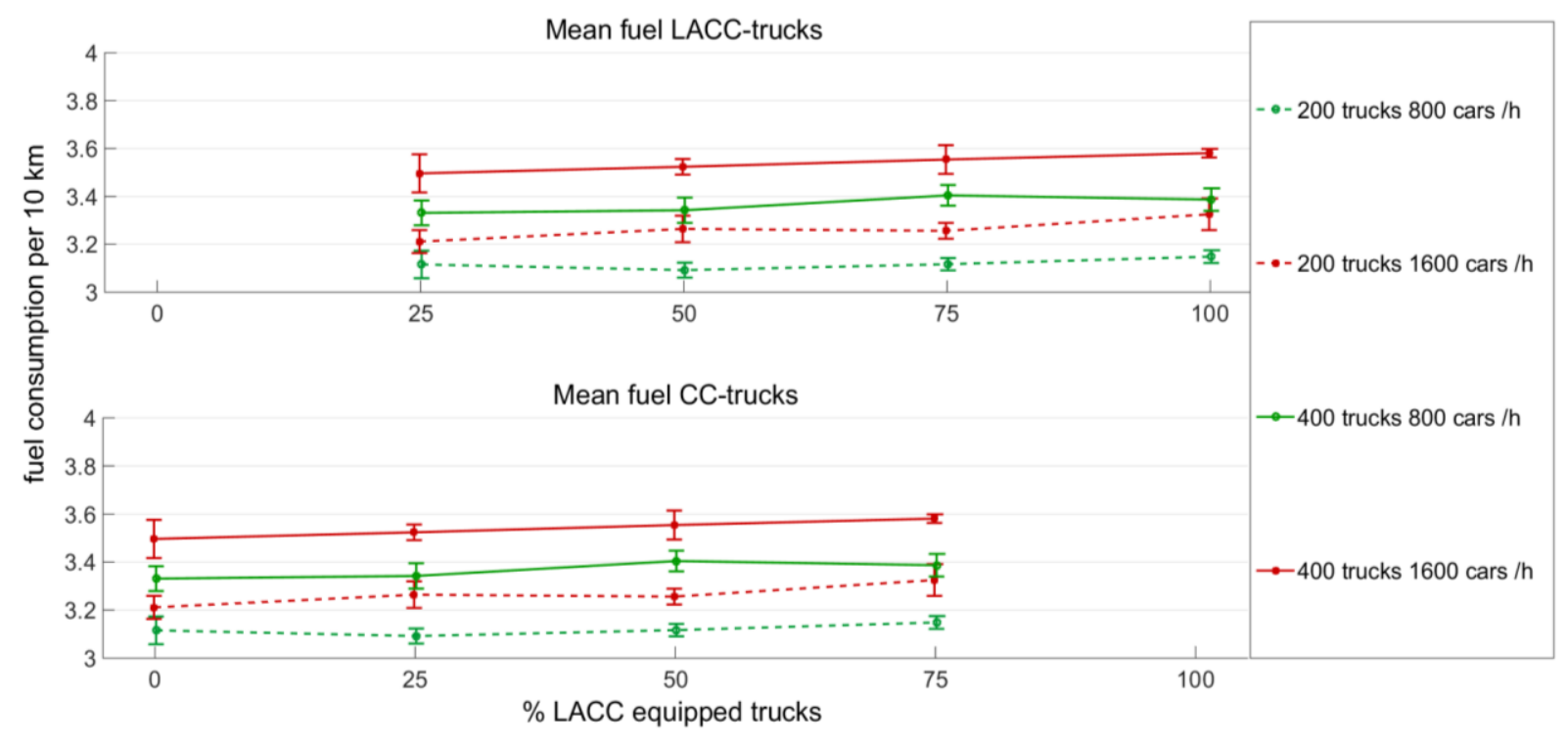

Figure 6.3. Comparison of fuel consumption for LACC-equipped trucks (top) and CC-equipped trucks (bottom) trucks for 4 traffic flows 
Table 6.7 Increase in mean fuel consumption for trucks by uniform vs normal distributed desired speed

\begin{tabular}{|c|c|c|c|c|c|}
\hline \multicolumn{6}{|c|}{ CC-trucks } \\
\hline $\begin{array}{l}\text { truck flow } \\
\text { (v/h) }\end{array}$ & $\begin{array}{c}\text { car flow } \\
\text { (v/h) }\end{array}$ & $\begin{array}{c}0 \% \text { LACC } \\
100 \% \text { CC }\end{array}$ & $\begin{array}{c}25 \% \text { LACC } \\
75 \% \text { CC }\end{array}$ & $\begin{array}{c}50 \% \text { LACC } \\
50 \% \text { CC }\end{array}$ & $\begin{array}{c}75 \% \text { LACC } \\
25 \% \text { CC }\end{array}$ \\
\hline 200 & 800 & $1.2 \%$ & $0.7 \%$ & $-0.4 \%$ & $0.8 \%$ \\
\hline 200 & 1600 & $4.1 \%$ & $4.9 \%$ & $4.1 \%$ & $5.7 \%$ \\
\hline 400 & 800 & $1.3 \%$ & $0.8 \%$ & $0.3 \%$ & $1.1 \%$ \\
\hline 400 & 1600 & $0.3 \%$ & $0.4 \%$ & $0.7 \%$ & $0.4 \%$ \\
\hline \multicolumn{6}{|c|}{ LACC-trucks } \\
\hline $\begin{array}{l}\text { truck flow } \\
\text { (v/h) }\end{array}$ & $\begin{array}{l}\text { car flow } \\
\text { (v/h) }\end{array}$ & $\begin{array}{c}25 \% \text { LACC } \\
75 \% \text { CC }\end{array}$ & $\begin{array}{c}50 \% \text { LACC } \\
50 \% \text { CC }\end{array}$ & $\begin{array}{c}75 \% \text { LACC } \\
25 \% \text { CC }\end{array}$ & $\begin{array}{c}100 \% \text { LACC } \\
0 \% \text { CC }\end{array}$ \\
\hline 200 & 800 & $5.3 \%$ & $5.8 \%$ & $5.0 \%$ & $3.8 \%$ \\
\hline 200 & 1600 & $8.6 \%$ & $7.7 \%$ & $8.5 \%$ & $8.3 \%$ \\
\hline 400 & 800 & $4.8 \%$ & $4.9 \%$ & $2.8 \%$ & $5.4 \%$ \\
\hline 400 & 1600 & $3.4 \%$ & $3.4 \%$ & $3.6 \%$ & $3.8 \%$ \\
\hline
\end{tabular}

The results in Figure 6.2, Figure 6.3, and Table 6.7 show a generally higher fuel use in the simulation experiments with uniform distributed desired speeds. A normal distribution of the desired speed causes less variations in desired speed, compared to a uniform distribution. This can imply that if the desired speed for LACC- equipped trucks varies in a wide range, it can cause extra accelerations and decelerations for other trucks, which results in higher fuel use. The trucks with higher desired speed might be more willing to overtake where the vehicles ahead have a lower desired speed which can result in even higher speed and fuel use.

\section{Comparing LACC- and CC-equipped trucks with trucks in free-flow traffic}

To find out how changes in traffic flow and the penetration rates of LACCtrucks influence the trucks' energy use, we created two reference scenarios when the trucks were driving in free-flow conditions. The total traffic flow in both scenarios is $200 \mathrm{v} / \mathrm{h}$ and all vehicles are trucks. The idea is to calculate the mean fuel used by the trucks in free-flow driving. The first reference scenario 
includes 200 LACC- equipped trucks and the second includes 200 CC-trucks with activated LACC/CC during the whole trip. In both graphs in Figure 6.4, fuel use increased more for CC-equipped trucks than LACC- equipped trucks compared to the fuel consumption in the reference scenario, with the exception for the no-car scenario (car flow $=0$ ).

Increase (\%) in fuel consumption compared to ref. trucks truck flow $=200 \mathrm{v} / \mathrm{h}$

30.00

25.00

20.00

15.00

10.00

5.00

0.00

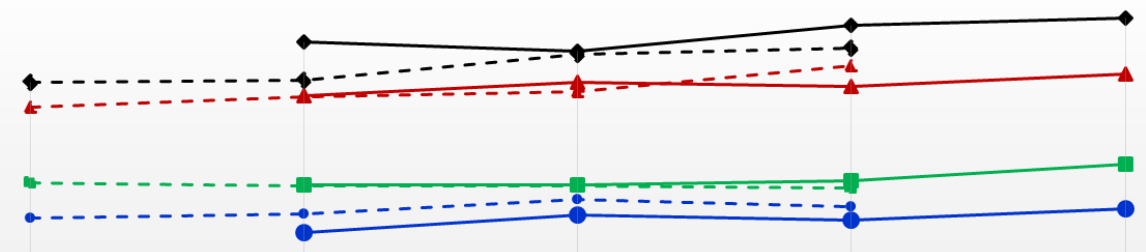

0\% LACC $\quad 25 \%$ LACC $\quad 50 \%$ LACC $\quad 75 \%$ LACC $\quad 100 \%$ LACC

Increase (\%) in fuel consumption compared to ref. trucks truck flow $=400 \mathrm{v} / \mathrm{h}$

35.00

30.00

25.00

20.00

15.00

10.00

5.00

0.00
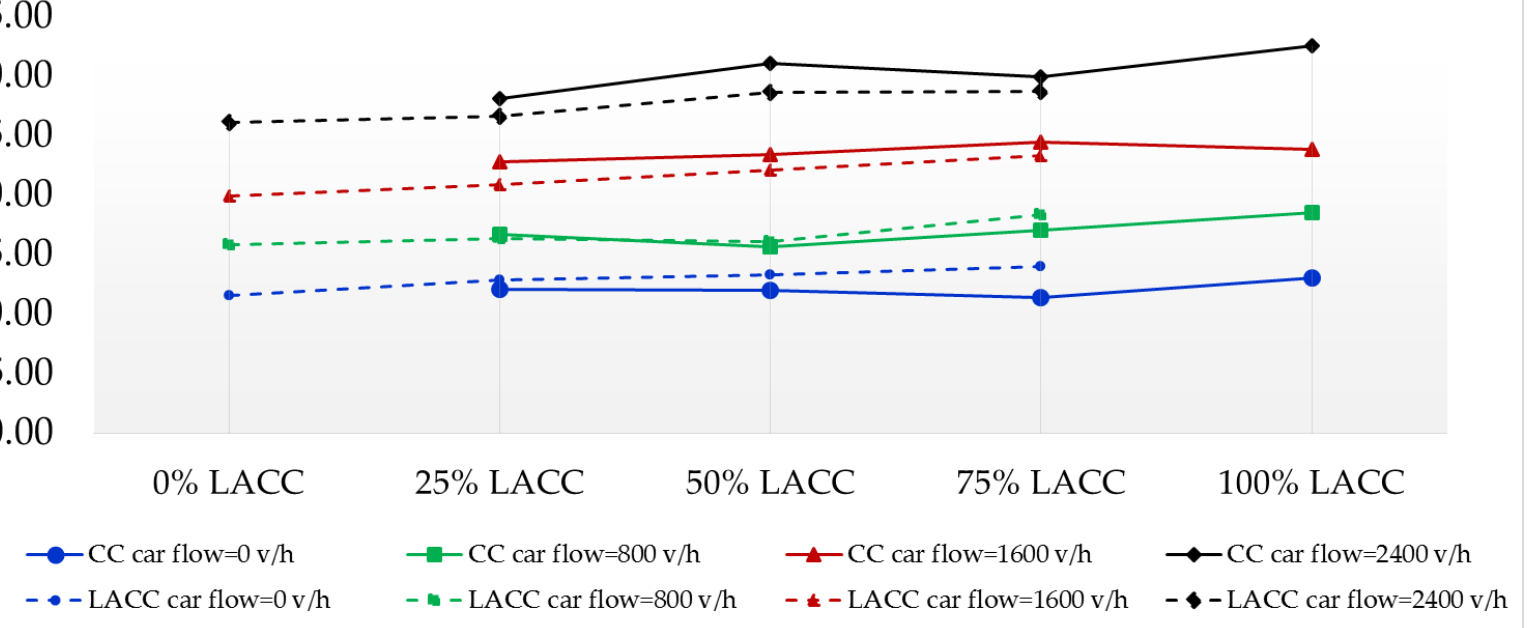

Figure 6.4. Increase (\%) in fuel consumption compared with free driving trucks

As expected, the increase in fuel use is more significant in higher traffic flow. However, an increase in truck flow seems to influence the level of fuel use more 
than an increase in car flow. This can be noticed for example by comparing the values in Table 6.8. In this table, the increase in the mean fuel consumption for LACC and CC-equipped trucks in the simulated scenarios, compared to a reference scenario without other surrounding vehicles is summarized. In most of the studied scenarios, it was observed that a higher truck flow causes a higher increase in fuel consumption, compared to a higher car flow. Since trucks may often be constrained by other trucks in traffic, it is expected that this happens more often when the number of trucks increases. When a truck is constrained by another truck, it may cause extra decelerations and accelerations that in turn cause higher fuel consumption.

Table 6.8 Degree of increase in mean fuel consumption compared with the reference scenario (free flow driving)

Increase (\%) in fuel consumption for LACC-equipped trucks compared to reference trucks

\begin{tabular}{|c|c|c|c|c|c|}
\hline $\begin{array}{c}\text { truck flow } \\
\text { v/h }\end{array}$ & car flow $\mathrm{v} / \mathrm{h}$ & $\begin{array}{c}25 \% \text { LACC } \\
75 \% \text { CC }\end{array}$ & $\begin{array}{c}50 \% \text { LACC } \\
50 \% \text { CC }\end{array}$ & $\begin{array}{c}75 \% \text { LACC } \\
25 \% \text { CC }\end{array}$ & $\begin{array}{c}100 \% \text { LACC } \\
0 \% \text { CC }\end{array}$ \\
\hline 200 & 0 & 5.67 & 6.03 & 7.32 & 6.66 \\
\hline 200 & 800 & 8.84 & 8.57 & 8.57 & 8.37 \\
\hline 200 & 1600 & 15.70 & 16.61 & 17.16 & 19.45 \\
\hline 200 & 2400 & 17.95 & 18.16 & 20.52 & 21.13 \\
\hline 400 & 0 & 11.59 & 12.87 & 13.26 & 13.95 \\
\hline 400 & 800 & 15.79 & 16.35 & 16.08 & 18.38 \\
\hline 400 & 1600 & 19.95 & 20.85 & 22.09 & 23.31 \\
\hline 400 & 2400 & 26.09 & 26.66 & 28.64 & 28.73 \\
\hline
\end{tabular}

Increase (\%) in fuel consumption for CC-equipped trucks compared to reference trucks

\begin{tabular}{|c|c|c|c|c|c|}
\hline $\begin{array}{c}\text { truck flow } \\
\mathrm{v} / \mathrm{h}\end{array}$ & car flow $\mathrm{v} / \mathrm{h}$ & $\begin{array}{c}0 \% \text { LACC } \\
100 \% \text { CC }\end{array}$ & $\begin{array}{c}25 \% \text { LACC } \\
75 \% \text { CC }\end{array}$ & $\begin{array}{c}50 \% \text { LACC } \\
50 \% \text { CC }\end{array}$ & $\begin{array}{c}75 \% \text { LACC } \\
25 \% \text { CC }\end{array}$ \\
\hline 200 & 0 & 4.35 & 5.91 & 5.40 & 6.44 \\
\hline 200 & 800 & 8.65 & 8.65 & 8.99 & 10.50 \\
\hline 200 & 1600 & 16.78 & 17.94 & 17.56 & 18.71 \\
\hline 200 & 2400 & 21.67 & 20.83 & 23.17 & 23.85 \\
\hline 400 & 0 & 12.10 & 11.96 & 11.39 & 13.00 \\
\hline 400 & 800 & 16.70 & 15.67 & 17.05 & 18.48 \\
\hline 400 & 1600 & 22.75 & 23.38 & 24.44 & 23.84 \\
\hline 400 & 2400 & 28.13 & 31.07 & 29.89 & 32.50 \\
\hline
\end{tabular}




\section{Fuel consumption for cars}

It is also interesting to analyse the fuel consumption of cars. The influence of variations in traffic flow and the penetration rate of LACC- equipped trucks in four scenarios, is illustrated in Figure 6.5. The results show that an increased penetration level of LACC-equipped trucks has no negative effect on the energy used by the cars. The average fuel consumption for cars decreases as traffic flow increases, which is surprising. It was expected that higher traffic flow would cause more accelerations and decelerations which results in higher fuel consumption. However, by considering the travel speed for cars, in Figure 6.11 we can notice that the mean travel speed for cars is significantly higher when traffic flow is low. This might influence the level of fuel consumption.

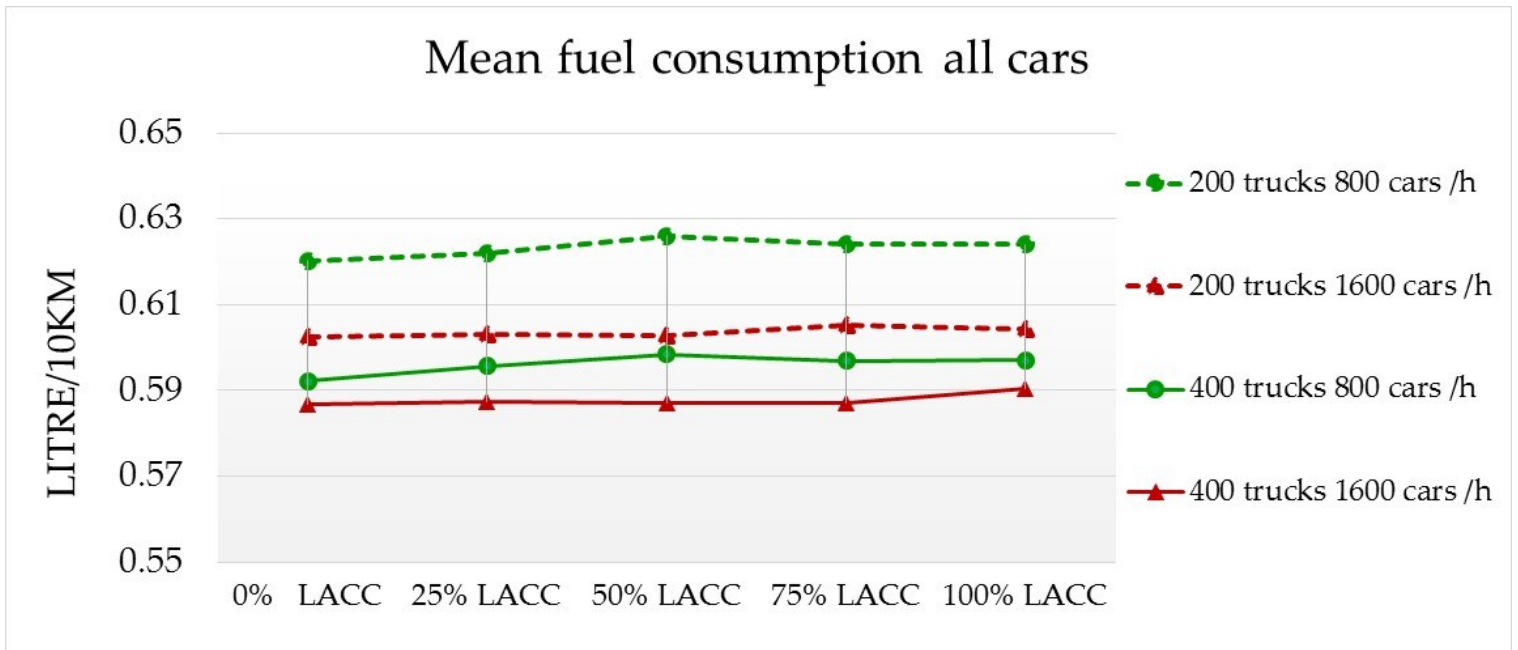

Figure 6.5. Fuel used by cars for four simulated scenarios

\subsubsection{Impacts on emissions}

In this section, the impacts of different traffic flow and penetration rate of LACC on traffic related emissions are presented. The PHEM model (Passenger Car and Heavy Duty Emission Model) Hausberger (2010) is used for emissions calculation. The input data for PHEM are the vehicle trajectories calculated in the simulation experiments. The emissions Hydrocarbon (HC), Carbon 
monoxide (CO), and Nitrogen Oxide (NOx) are calculated for the following four scenarios:

1. Truck flow is $200 \mathrm{v} / \mathrm{h}$ and car flow is $800 \mathrm{v} / \mathrm{h}$

2. Truck flow is $200 \mathrm{v} / \mathrm{h}$ and car flow is $1600 \mathrm{v} / \mathrm{h}$

3. Truck flow is $400 \mathrm{v} / \mathrm{h}$ and car flow is $800 \mathrm{v} / \mathrm{h}$

4. Truck flow is $400 \mathrm{v} / \mathrm{h}$ and car flow is $1600 \mathrm{v} / \mathrm{h}$

Figure 6.6 shows the mean value of traffic related emissions generated by all trucks in the simulated traffic. The calculations of Hydrocarbon (HC), Carbon monoxide (CO) and Nitrogen monoxide (NOx) are included in the analysis. In calculating traffic related emissions, Carbon dioxide $\left(\mathrm{CO}_{2}\right)$ emissions are relatively straightforward to estimate. The level of $\mathrm{CO}_{2}$ depends on two factors, the type of fuel and the level of used fuel. For all simulated trucks, the type of fuel is assumed to be diesel while the simulated cars use either petrol or diesel. Since the proportion of diesel and petrol cars is the same in all simulation scenarios, a comparison between the total levels of fuel consumption for cars would be reliable. The level of generated $\mathrm{CO}_{2}$ can therefore be perceived by observing the results concerning fuel consumption.

In general, for all scenarios, emissions increase with increased traffic flow. An increased car flow seems to have more influence than truck flow on emission increase. However, a comparison between the case with no LACC- equipped trucks and when $25 \%$ of trucks are equipped with LACC, indicates a significant improvement and emissions are reduced. The same reduction can be observed when the penetration level of LACC- equipped trucks increases from $75 \%$ to $100 \%$.

Comparison between the outcomes related to LACC- and CC-equipped trucks shows that emissions increase when traffic flow increases, and CC-equipped trucks are generally less influenced by traffic flow, see appendix II. Emissions generated by LACC-equipped trucks are significantly less than emissions made by CC-equipped trucks in all scenarios. A clear difference can be seen between scenario 1 and other scenarios for $\mathrm{CO}$ and NOx emissions. This means that the level of CO and NOx emissions made by both LACC and CC-equipped trucks, are strongly influenced by traffic flow. It is surprising that increased car flow seems to have more effect on the level of emissions for trucks, compared to 
increased truck flow. A larger difference can be seen in the results shown in Figure 6.6 between the blue and red curves compared to the difference between the red and black curves. The truck flow in scenario 1 and 2 is the same $(200 \mathrm{v} / \mathrm{h})$ while the truck flow in scenario 4 is double the truck flow in these scenarios (400 $\mathrm{v} / \mathrm{h})$. This can indicate that, when car flow increases from 800 to 1600, the level of emissions for trucks increases significantly, compared to when truck flow increases from 200 to 400 .

-200 trucks 800 cars $/ \mathrm{h} \rightarrow-200$ trucks 1600 cars $/ \mathrm{h} \rightarrow-400$ trucks 800 cars $/ \mathrm{h} \rightarrow 400$ trucks 1600 cars $/ \mathrm{h}$

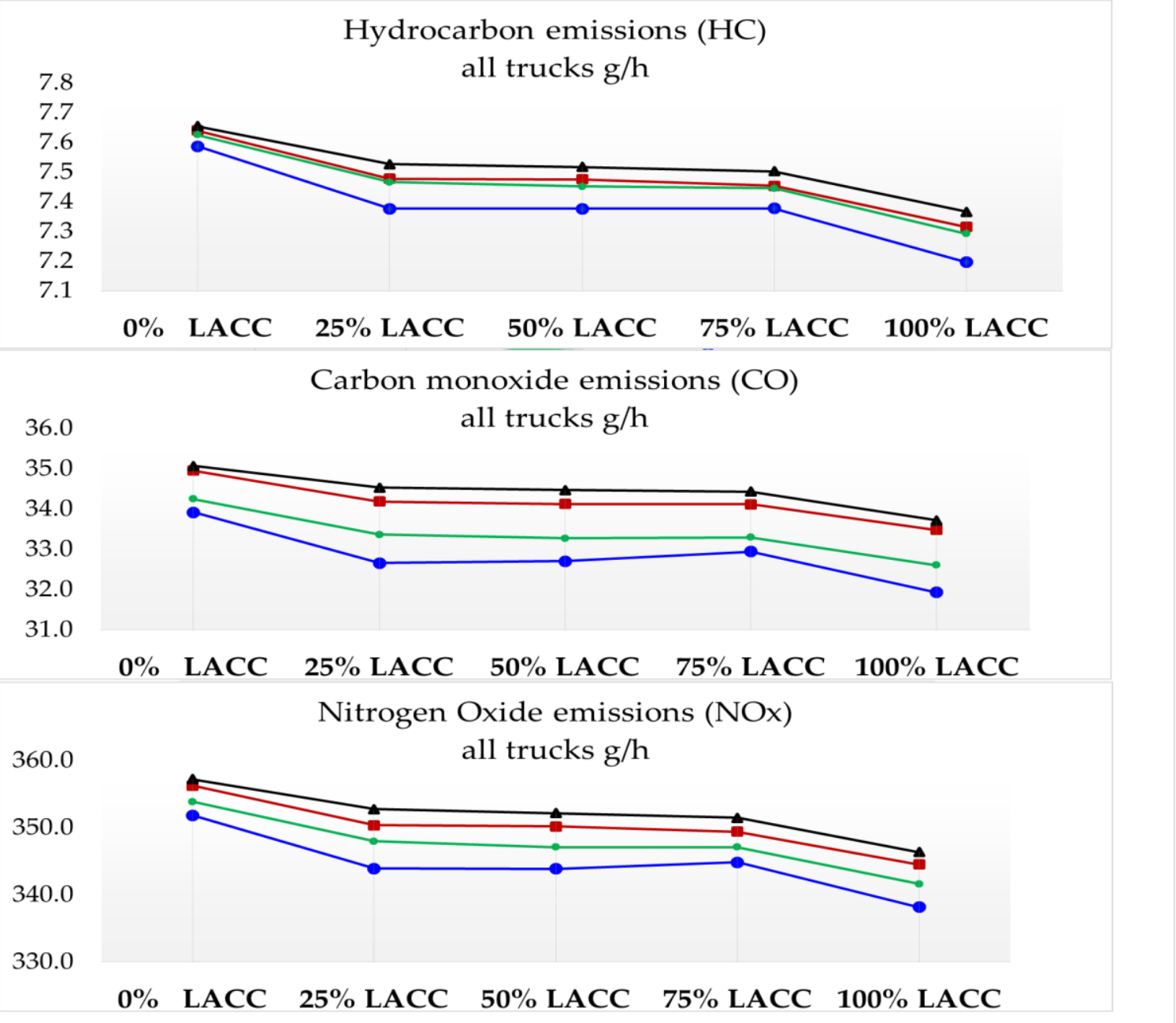

Figure 6.6. Traffic related emissions ( $\mathrm{HC}, \mathrm{CO}$ and NOx) made by all trucks in different traffic flow and penetration rates of LACC-trucks

The traffic related emissions generated by cars are also interesting to study. In Figure 6.7 the mean value of the emissions are plotted for the cars in the four 
simulation scenarios. The different penetration rates of LACC- equipped trucks seems not have any effect on the level of emissions made by cars. We notice a higher level of emissions for scenario 2 compared to scenario 4 , in spite of the higher truck flow in scenario 4 . This can be explained by the higher travel speed for the cars when the trucks flow is lower.

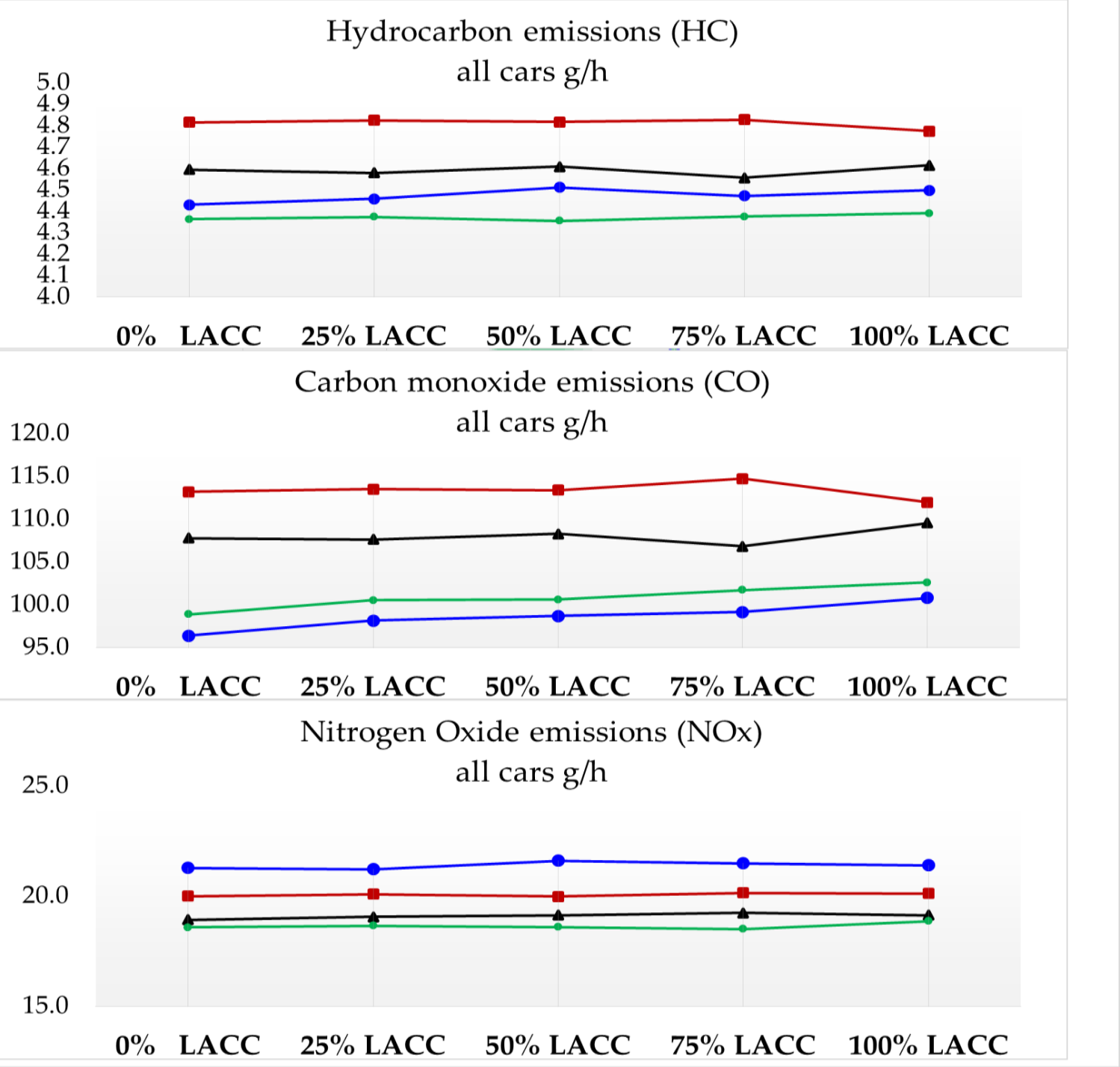

Figure 6.7. Traffic related emissions (HC, $\mathrm{CO}$ and NOx) made by all cars in different traffic flow and penetration rates of LACC-trucks 
The same effect in the measured HC and NOx, is noticed for scenario 1 and 3 with equivalent car flow and different truck flow. But, the level of $\mathrm{CO}$ is higher in scenario 3 than scenario 1 . This reveals that in lower traffic flow, an increase of truck flow causes a higher level of $\mathrm{CO}$ emission.

\subsection{Impacts on traffic efficiency}

In this section, the impacts on traffic efficiency will be presented and discussed. The measures that have been selected are travel speed and time.

\subsubsection{Impacts on travel speed}

Since LACC affects the vehicle's speed, it is therefore interesting to study the travel speed of the trucks in different scenarios. Figure 6.8 shows the comparison between the mean travel speed for all trucks with respect to the different car and truck flow as well as the different penetration rates. The solid lines indicate the mean speed for all trucks in the scenarios when the truck flow is $400 \mathrm{v} / \mathrm{h}$ and the dashed lines indicate the mean speed for all trucks in scenarios when the truck flow is $200 \mathrm{v} / \mathrm{h}$. Through analysis of Figure 6.8, it is evident that when total flow increases, travel speed decreases. This result was expected, since when total flow increases, vehicles constrain each other. Therefore, the vehicles become unable to maintain their desired speed for the whole duration of the journey. The difference in the curves, showing the space mean speed of the vehicles, becomes clearer the higher the flow. It is interesting to evaluate how an increasing number of cars affects the travel speed of the trucks. Comparing the curves for two scenarios with the same number of trucks and different number of cars will aid in this evaluation. Generally, the space between the solid lines is larger than the space between one solid and one dashed line with the same color. This means that in the studied scenarios the increase in car flow has more effect on the travel speed. However, car flow in the scenarios differ by $800 \mathrm{v} / \mathrm{h}$ while trucks flow differ by $200 \mathrm{v} / \mathrm{h}$. An increase with $200 \mathrm{v} / \mathrm{h}$ in car flow would probably not affect the results in the same way. 


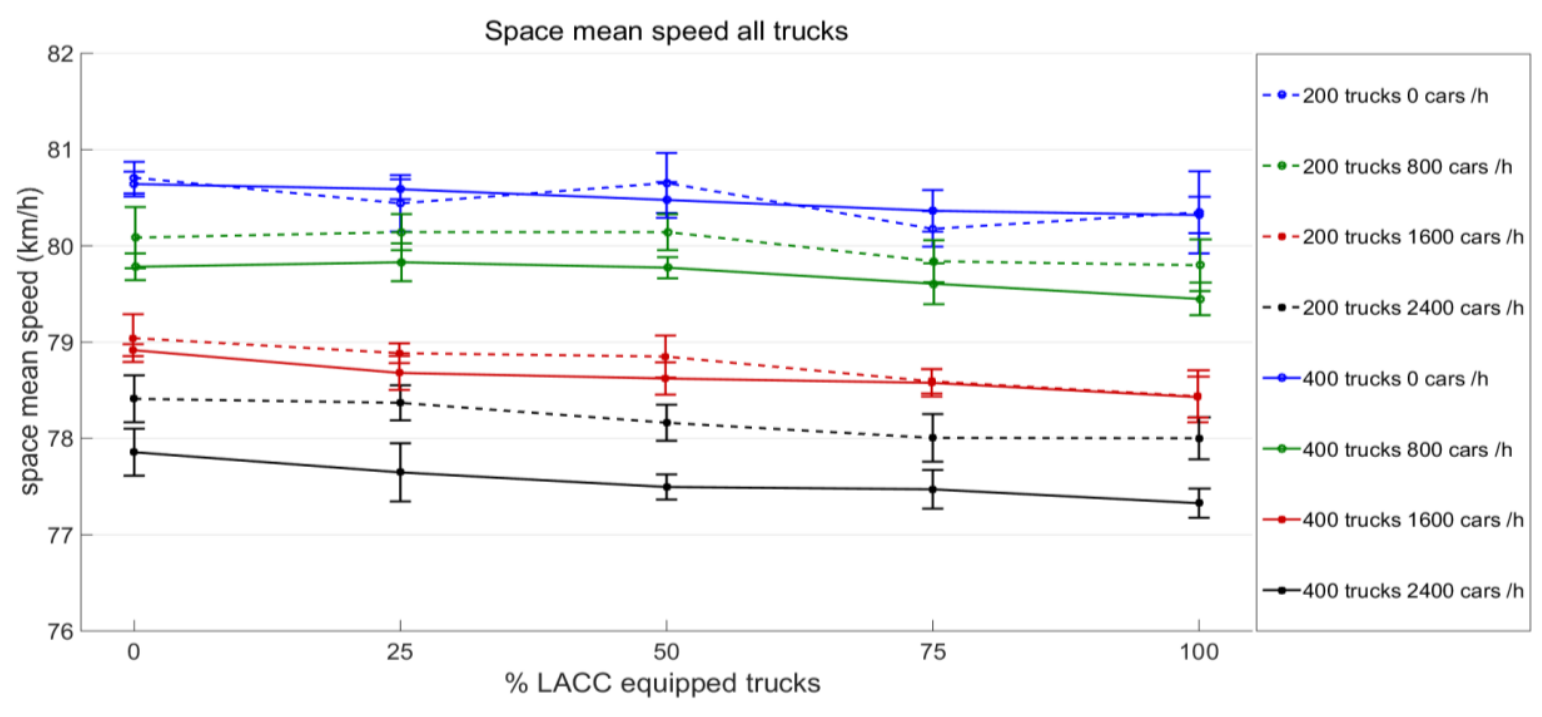

Figure 6.8. Comparison of mean travel speed for all trucks in 8 scenarios

Figure 6.9 shows the comparison between the mean travel speeds for all trucks that are assigned normal distributed desired speeds. The figure shows the mean travel speed for all trucks with respect to the different car and truck flow as well as the different penetration rates. When comparing Figure. 6.8 and 6.9, we notice that the mean travel speed for the scenarios with different distribution of desired speeds do not differ significantly. A clear increase can be seen in the mean travel speed for the trucks with normal distributed desired speed in the scenarios with 1600 cars. In these scenarios, traffic is more dense compared to the other scenarios with 800 cars. A lower mean travel speed can indicate that the trucks constrain each other more if desired speeds are more varied, which can be observed in Figure 6.8. Different penetration rates of LACC- equipped trucks seems not to have influence on mean travel speed in the scenarios shown in Figure 6.9. 


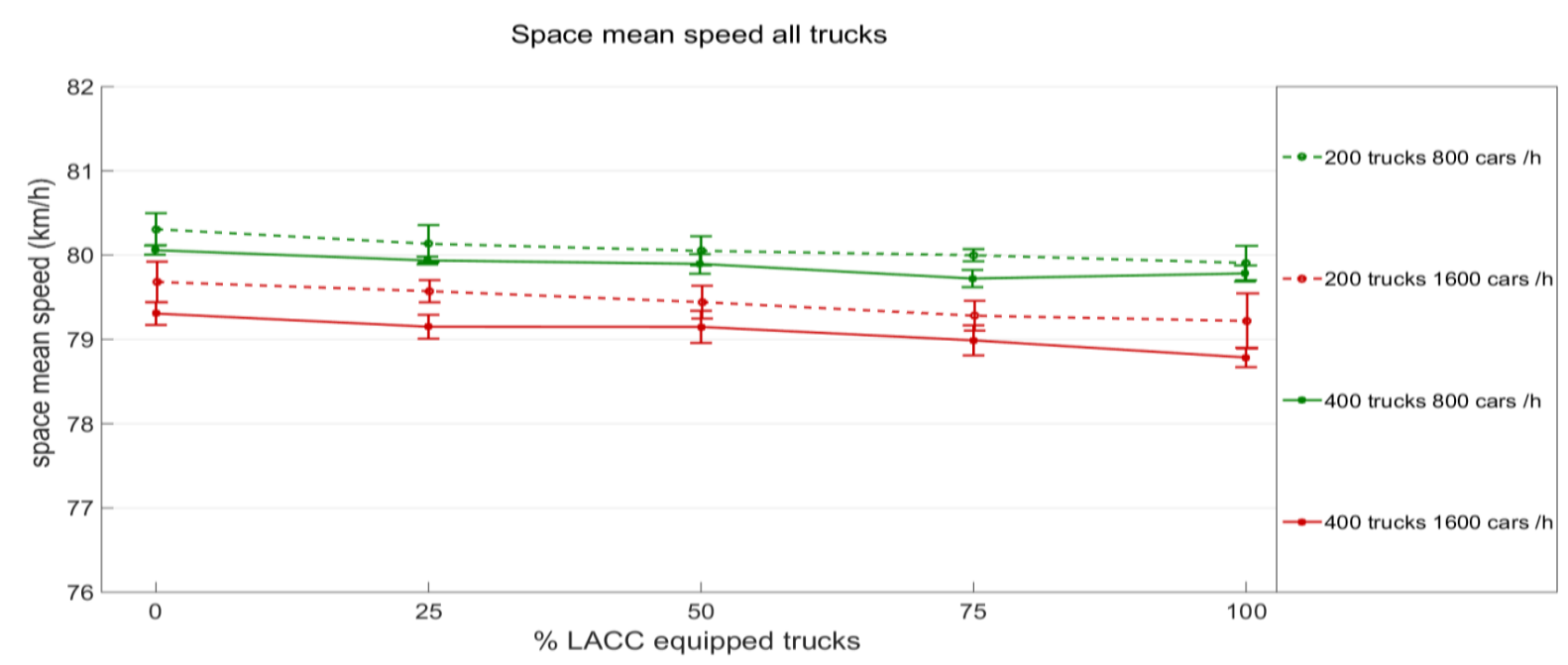

Figure 6.9 Comparison of travel speed for all trucks with normal distributed desired speed

Figure 6.10 shows results for the mean travel speed for CC and LACC- equipped trucks separately. No significant difference can be observed between CC and LACC-equipped trucks in any scenario. An expected increase in the mean travel speed is noticed for both CC- and LACC- equipped trucks, when traffic flow increases. However, an increase in car flow seems to influence the mean travel speed, more than an increase in truck flow. This can be seen by comparing the solid curves (or the dashed curves). The solid curves indicate the scenarios where truck flow is $400 \mathrm{v} / \mathrm{h}$ and car flow varies. The space between the solid curves is greater than the space between a dashed curve and a solid curve with the same colour. This trend is noticed in Figures 6.8 and 6.9 as well. 


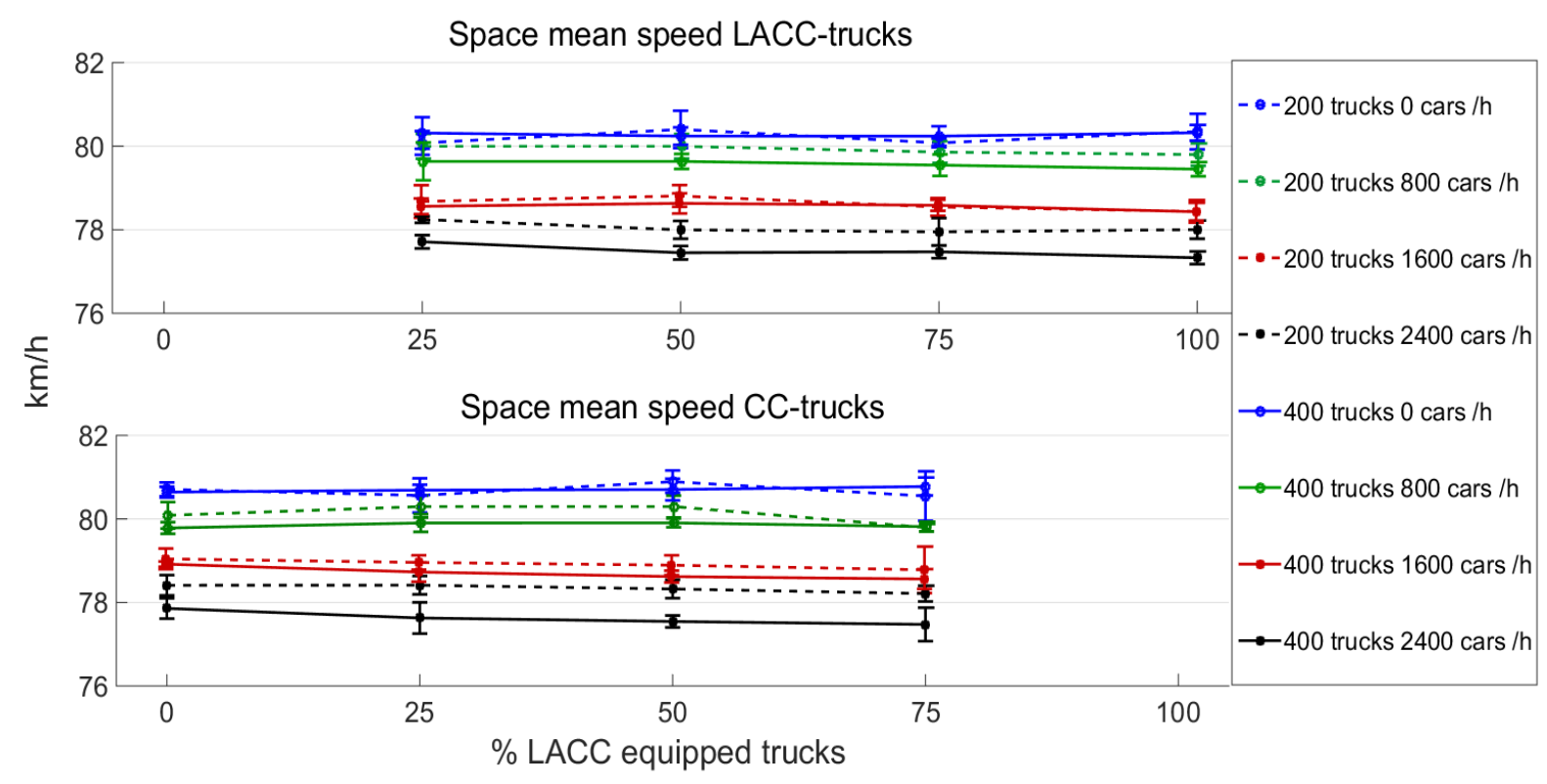

Figure 6.10 Comparison of travel speed for LACC-equipped (top) and CC-equipped trucks (bottom) for 8 scenarios

Travel speed for cars is another performance indicator that is calculated in order to study LACC- equipped trucks impacts on traffic efficiency. Figure 6.11 shows the travel speed for cars in the 6 scenarios that include cars. Different proportion of LACC- equipped trucks in the simulated traffic does not seem to influence the cars' travel speed. However, both car and truck flow affect the cars' travel speed. We can notice that the space between the red dashed and solid green line is very small. This means that 800 more cars per hour have the same effect as 200 more trucks per hour. The scenario indicated by the green solid line, includes 200 more trucks and 800 less cars than the other scenario indicated by the dashed red line. 
Average travel speed all cars

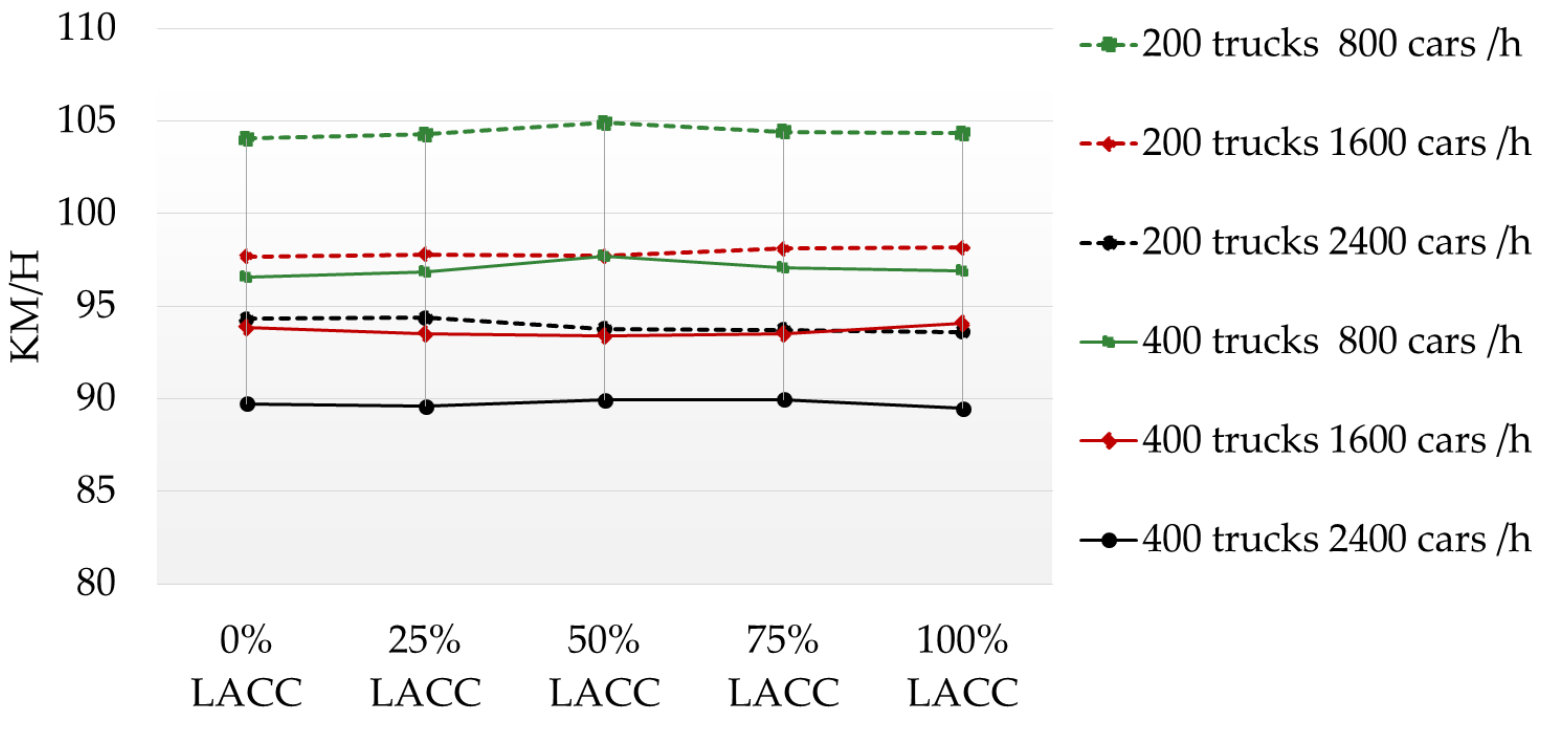

Figure 6.11 Comparison of travel speed for cars for scenarios including cars

\subsubsection{Impact on travel time}

The travel time measured for all trucks is given in Figure 6.12 These graphs confirm the results of Figure 6.8 with the exception that travel time increases with increased traffic flow. When comparing the travel time between different penetration rates of LACC- equipped trucks, we see that travel time increases as the number of LACC- equipped trucks increases in each scenario. 


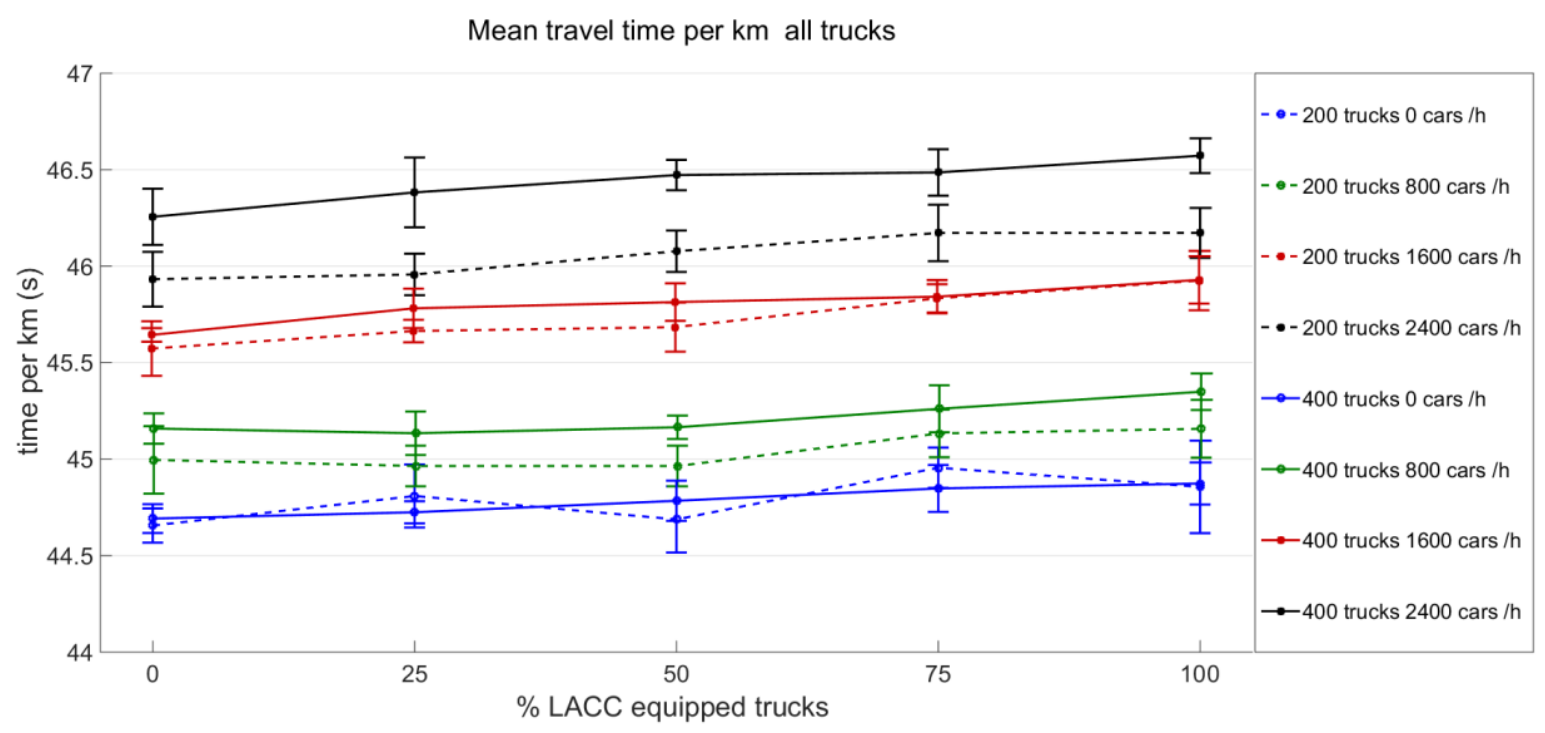

Figure 6.12 Comparison of travel time for all trucks for 8 scenarios

Results concerning the travel time for all trucks with normal distributed desired speed are displayed in Figure 6.13. The results show lower travel time for these scenarios compared to the scenarios presented in Figure 6.12. The trucks seem to take more time to drive the same distance when the desired speeds vary widely, and the difference between the scenarios with different traffic flow is more clear. We can see this trend in the figure by comparing the difference between the solid curves (the red and the green one) or the difference between the dashed curves. 
Mean travel time per km all trucks

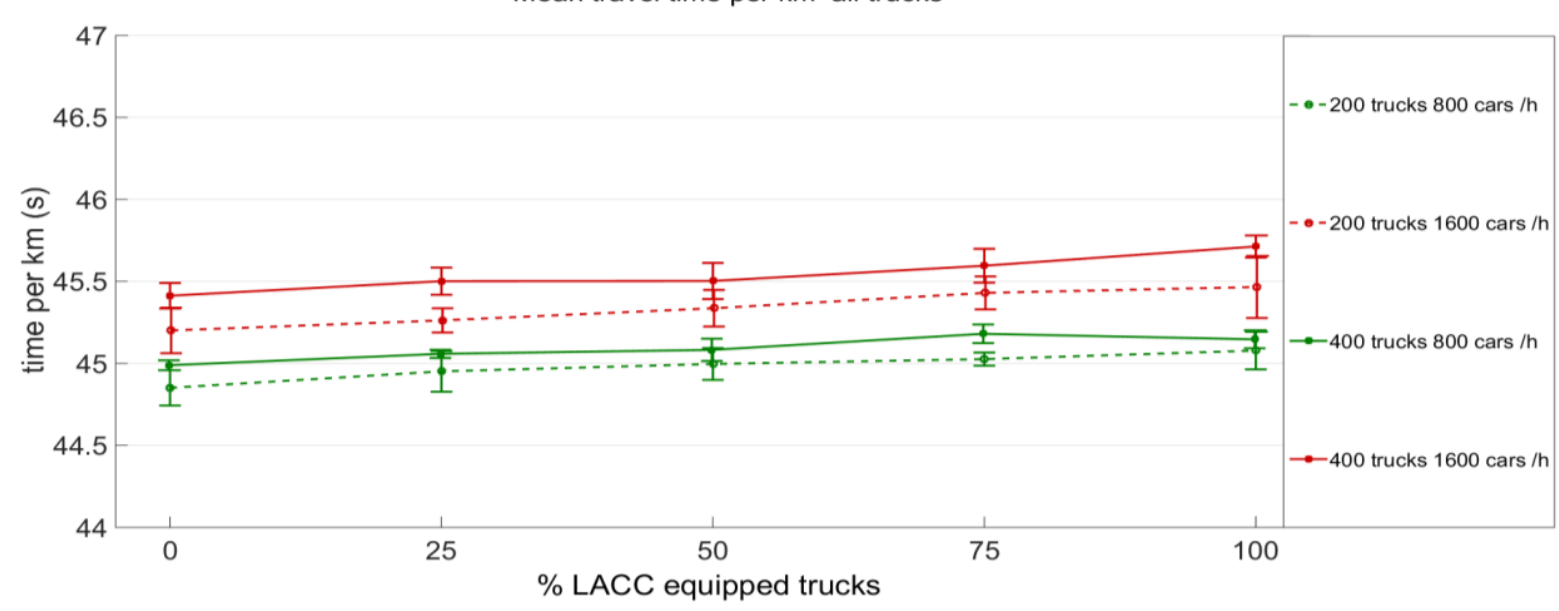

Figure 6.13 Comparison of travel time for all trucks with normal distributed desired speed

The travel time for LACC- and CC- equipped trucks is displayed in Figure 6.14. The results show no significant difference between the measured travel times between different penetration rates of LACC- equipped trucks. LACCequipped trucks seem to have longer travel time than CC-equipped trucks. However, this difference is not especially large.

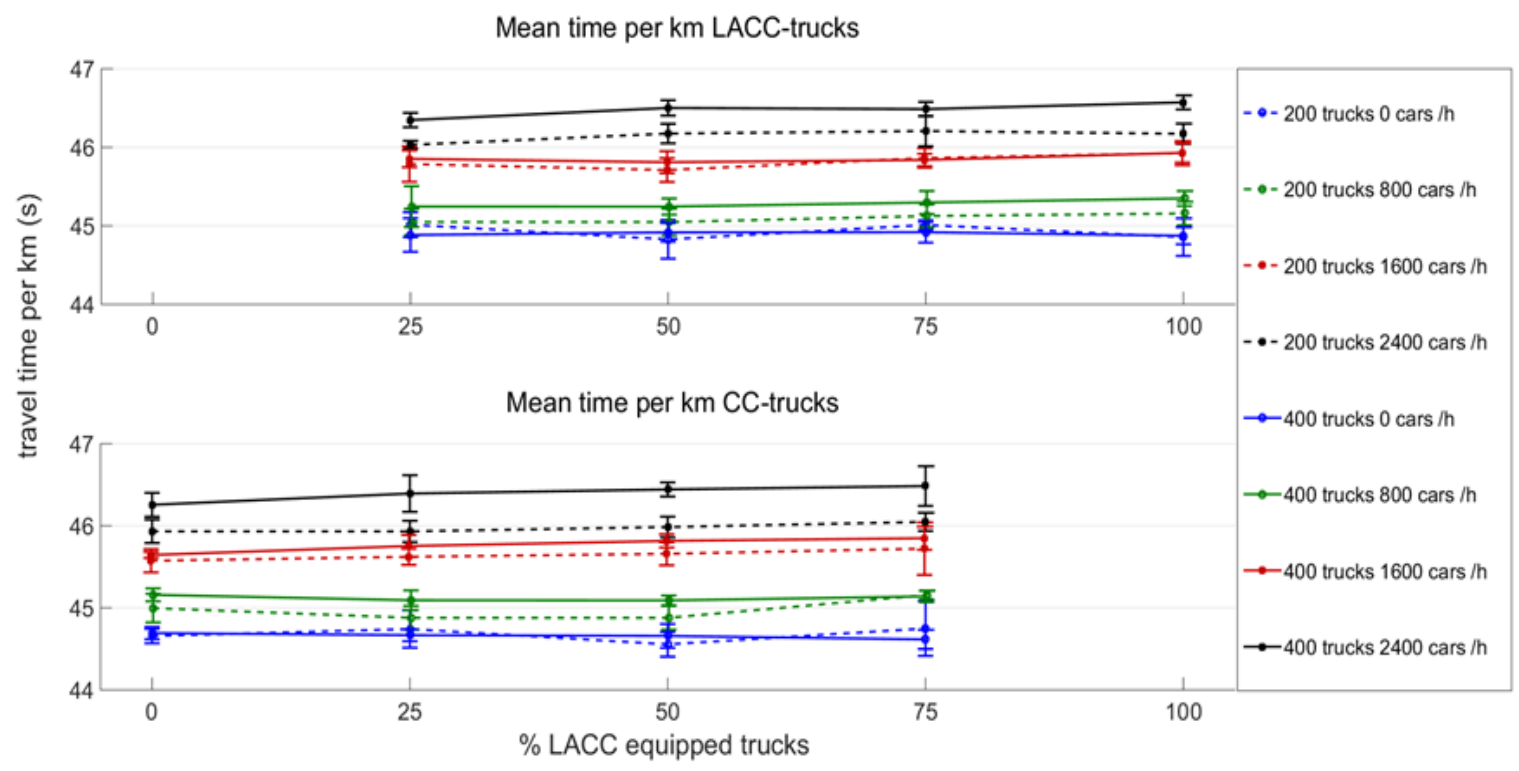

Figure 6.14 Comparison of travel time for LACC- equipped trucks (top) and CCequipped trucks (bottom) for 8 traffic flows 
The average travel time (per kilometre) for all cars are plotted in Figure 6.15. These graphs confirm the results of Figure 6.11 with the exception that travel time increases with increased traffic flow. The observed effect of increasing truck or car flow is also noticed in these results, i.e. 800 more cars per hour have same effect as 200 more trucks per hour. When comparing the travel time between different penetration rates of LACC- equipped trucks, we do not see any significant variation.

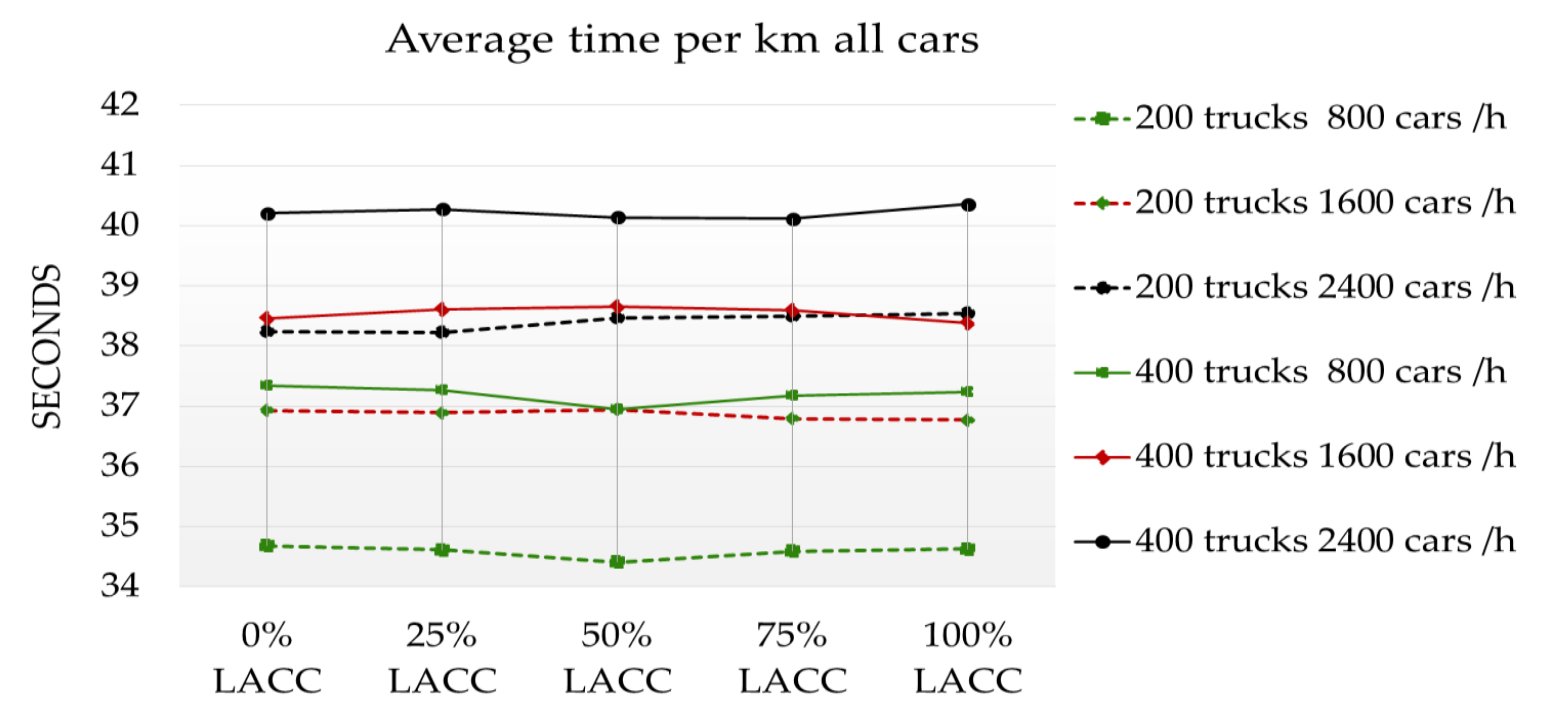

Figure 6.15 Comparison of travel speed for cars

\subsection{LACC and CC utilization}

In this section, the utilization of LACC and CC is investigated. It is interesting to compare the utilization of LACC and CC to see how different flows affect activation and reactivation of the systems, but also to see how such utilization affects the other measured indicators. The portion of LACC- and CC-equipped trucks that have activated LACC/CC at least $50 \%$ of the travel distance is shown in Figure 6.16. 


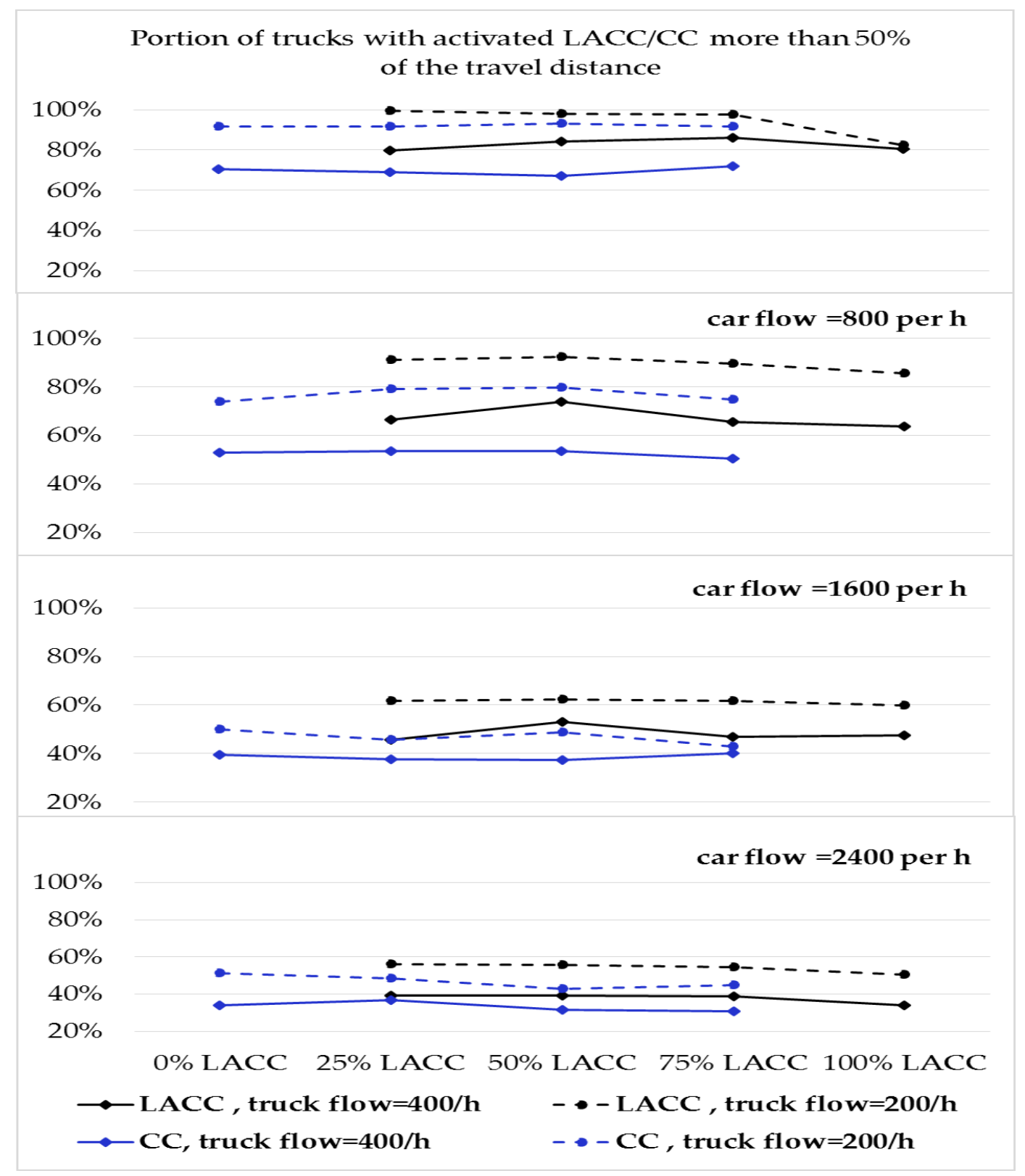

Figure 6.16 Portion of trucks that keep LACC or CC activated at least $50 \%$ of the travel distance

Figure 6.16 shows that as traffic flow increases, the number of trucks that have the system activated decreases. However, it seems that a larger number of LACC trucks choose to keep the system active for a minimum of $50 \%$ of the travel distance. Different penetration rates of LACC seem not to influence the result. More detailed figures are presented in Appendix II. The figures show the 
portion of trucks that have activated LACC/CC with respect to the portion of the travel distance in different traffic flows.

\subsection{Discussion and conclusions}

The influence of variations in car flow, truck flow, and the penetration rates of LACC-equipped trucks is studied. A motorway section with two lanes, containing cars and trucks, different traffic flow and portions of LACCequipped trucks is simulated and a set of performance indicators is measured. Several graphs and tables are presented and analysed in order to examine the hypotheses described in 6.1.1. Differences in fuel consumption, emissions, travel time, travel speed and utilization of LACC/CC is measured and analysed. By analysing the results and testing the hypotheses, we can consider the following conclusions:

- LACC will have a positive effect on fuel consumption and on traffic related emissions for LACC-equipped trucks.

The results concerning mean fuel consumption and the traffic related emissions revealed a positive effect of LACC for LACC-equipped trucks. However, a significant improvement can not be seen when the penetration level of LACC-equipped trucks increases.

- An increased penetration rate of LACC will not have negative effects on emissions for LACC-and CC-equipped trucks.

Evaluation of results related to emissions, revealed a clear improvement when comparing $0 \%$ and $100 \%$ penetration level of LACC-equipped trucks. We can also observe a significant decrease in emissions between $0 \%$ and $25 \%$ as well as between a $75 \%$ and $100 \%$ level of LACC-equipped trucks. This can indicate that LACC-equipped trucks in traffic reduce the level of traffic related emissions and the maximum effect will be achieved when all trucks have LACC. An increased penetration rate of LACC will not have any effect on energy use or emissions for the cars. 
Higher penetration rates of LACC show no negative effect on HC and NOx car emissions. An increase in the level of $\mathrm{CO}$ made by cars, can be noticed when the portion of LACC- equipped trucks increases.

- LACC will increase the travel time for LACC-equipped trucks. LACC will not have a negative effect on travel time for cars and CC-equipped trucks.

As expected, LACC shows no negative effect on traffic efficiency, but a small increase in travel time can be noticed. LACC-equipped trucks seem to have somewhat higher travel time than CC-equipped trucks, but the difference is not significant.

- LACC will have less effect for LACC-equipped trucks in higher traffic flow situations and in denser traffic conditions.

Generally, LACC shows less effect in denser traffic conditions, while the proportion of LACC-equipped trucks does not show significant effect on travel time for cars or trucks.

- LACC will have less effect for LACC- and CC- equipped trucks, if drivers' desired speed varies considerably.

The effects of variations in the truck drivers' desired speed are studied through comparing the measured performance indicators for the simulated scenarios including trucks assigned with normal or uniform distributed desired speeds. The analyses show that LACC has more effect on the measured performance indicators when the drivers' desired speed varies considerably. 


\section{Chapter 7}

\section{Discussion and future research}

The development of Advanced Driver Assistant Systems (ADAS) has become an important element in the traffic system. Focus has mainly been on driver support systems for increasing traffic safety and comfort, but eco-driving ADAS has been given more attention recently. Eco-driving ADASs are more interesting for trucks and heavy vehicles, especially in terrain with varying topography, since the driving style and heavy loads, affect fuel consumption more significantly than for cars. The fuel cost is a considerable proportion of the total vehicle cost for transport companies and $\mathrm{CO}_{2}$ emissions are directly affected by the fuel consumption. An Eco-driving ADAS is supposed to decrease fuel consumption and emissions for the equipped vehicle. However it may also affect the surrounding vehicles' driving behaviour, e.g. since the equipped vehicle may accept geometric deceleration on uphill slopes when 
other drivers accelerate or keep a constant speed. Thus the effects on the overall traffic system should be evaluated before implementing a driver support system in the real traffic system. The effects of an ADAS on traffic safety, environmental impact and level-of-service for individual drivers, should be measured and evaluated irrespective of the aims of the ADAS. In that context, it is essential to aggregate the individual driver behaviour to the traffic system level.

The research presented in this thesis has focused on evaluation of Advanced Driver Assistance Systems (ADAS), more precisely different types of fuel controllers, focusing on fuel minimizing cruise controllers for trucks and more specifically, the Look Ahead Cruise Control (LACC). We pointed out the importance of a combined framework in order to achieve enhanced results. The overall aim of this thesis has been to quantify the fuel consumption and emissions generated by cars and trucks, when some of the trucks are equipped with LACC. By studying different flows of cars and trucks and various penetration levels of LACC-equipped trucks, we determined the effect on fuel consumption and the generated traffic-related emissions. In addition, the resulting traffic performance and driving behavior were analyzed.

Simulation experiments were carried out to observe the LACC impacts on the traffic system with respect to penetration rate, traffic density and variation in the desired speed. A driver model was developed to capture the changes in driving behaviour in a LACC-equipped truck. Environmental effects of the LACC were estimated using emission calculations, and the results were analysed.

Generally, LACC shows less effect in more dense traffic conditions, while the proportion of LACC-equipped trucks does not show significant effect for cars or trucks. Results from the evaluation indicate that LACC can contribute to improvements in the level of traffic-related emissions. Comparison between the improvement in fuel consumption for LACC-equipped and CC-equipped ${ }^{6}$ trucks show that LACC is able to decrease the total fuel consumption in all scenarios but the benefit for the equipped trucks seems to drop upon low car flow, because the trucks can drive with activated LACC and CC on a longer

${ }^{6}$ Cruise Control, for more detailed description see Section 2.3 
stretch of the road and the benefit compared with free driving is not so remarkable. The simulation results show that LACC-trucks generally have a lower mean speed compared with CC-trucks. When the traffic is dense, the trucks may constrain the other trucks and this may causes an increase in fuel consumption for all trucks. However, the utilization of the LACC/CC decrease clearly when the traffic is dense and the results of the fuel consumption may not be relevant in comparing with the other scenarios. The results show that as traffic flow increases, the number of trucks that have the system activated decreases. However, it seems that a larger number of LACC- equipped trucks choose to keep the system active, compared to CC-equipped trucks.

In order to study if the variations in the desired speeds for the trucks influence the measured indicators, we conducted simulation experiments where the variations in the desired speed are small or large. A comparison between the simulation results for scenarios including trucks with small variations in desired speed show a generally higher fuel use in the simulation experiments compared to a simulation when the trucks' desired speeds have large variations. This can imply that if the desired speed for trucks vary in a wide range, these trucks can cause extra accelerations and decelerations for other trucks which results in a higher fuel consumption. The trucks with higher desired speed might be more willing to overtake where the vehicles ahead have a lower desired speed which can result in even higher speed and fuel consumption.

In order to study the effects on the cars, the fuel consumption and emissions related to the cars in the simulation experiments were considered. The results show that an increased penetration level of LACC- equipped trucks has no negative effect on the energy used by the cars. The average fuel consumption for cars decreases as traffic flow increases, which is surprising. It was expected that the higher traffic flow would cause more accelerations and decelerations which results in higher fuel consumption. However, by considering the travel speed for cars, we can notice that the mean travel speed for cars is significantly higher when traffic flow is low. This might influence the level of fuel consumption. When the truck flow is low, Hydrocarbon emissions (HC) and Carbon monoxide emissions (CO) generated by cars increase more with respect to increased car flow. A higher car flow seems to have an opposite effect on the level of Nitrogen Oxide emissions (NOx) generated by cars. 
The mean travel speed for all trucks with respect to the different car and truck flow as well as the different penetration rates were compared. Through analysis of the results, it is evident that when total flow increases, travel speed decreases. This result was expected, since when total flow increases, vehicles constrain each other. Therefore, the vehicles become unable to maintain their desired speed for the whole duration of the journey. Equivalent analysis for cars shows that different proportion of LACC- equipped trucks in the simulated traffic seem not to influence the cars' travel speed.

We have conducted the simulation experiments for a motorway since this is the type of road where cruise controllers are most frequently used. We have assumed that all trucks are Scania long-haul trucks, and that all trucks have the same engine power, vehicle length and weight. All trucks are assumed to be equipped with CC or LACC to maintain the speed and one downhill speed control (DHSC) that uses the auxiliary brake systems to maintain a desired downhill set-speed.

The research carried out in this thesis indicates that there are many interesting questions that need further investigation in future research studies. An interesting question is how we can apply the evaluation framework presented in this thesis for other ADAS. One of the main contributions of this study was the interconnection of an external system into an existing traffic simulation model. One of the advantages of this framework was to avoid implementing the functionality of the system in the traffic simulation model. Having a complete vehicle simulation helped us to capture a reliable simulation of the vehicle dynamics and the ADAS. Another benefit of the interconnected model was to be able to simulate several in-vehicle systems at the same time. Considering the increasing number of these systems in the traffic, and the complexity of modelling several in-vehicle systems in a traffic simulation model, this approach can be applied. The major drawback is probably that the verification and validation of the model is time consuming and requires deliberate data collection and behavioural studies. However, this is more or less always the case when conducting complex microscopic traffic simulation investigations.

The Field Observation Test (FOT) carried out here was limited to three trucks that were driving the same route. We did not have any data to estimate the headway to the vehicles in the adjacent lane or the vehicle behind. Clearly, 
modelling the driving behaviour of ADAS-equipped trucks would be more reliable if we had information about the preceding vehicle's speed, the time headway, and traffic flow. This kind of data could help us to set accurate parameters as used in our driver sub-model. Regarding evaluation of an ADAS in a specific traffic condition, this kind of data collection can be useful.

Other potential future research is to study the level-of-service for traffic systems including ADAS-equipped vehicles. We have analysed the travel speed and travel time in this thesis. Other measures such as safety, freedom to manoeuvre, and traffic interruptions can be measured and analysed. Traffic simulation models are suitable tools for studying level-of-service. By extending the existing traffic simulation models to include ADAS-equipped vehicles, it is possible to simulate traffic systems that include vehicles equipped with various type of ADAS. 
Chapter 7. Discussion and future research 


\section{Appendix I}

\section{Focus Group Study}

This appendix presents the set-up for the Focus Group Study and summarizes the findings. The FGS is done in order to give input to traffic simulation modelling of cruise controllers. 
The Focus Group Study (FGS) is based on qualitative data analysis revealed from discussions in three focus groups. The FGS experiment were led by two discussion leaders from VTI who guide the discussions, record the discussion digitally and summarize the answers and comments. The experiment was conducted in June 2013.

\section{Purpose:}

The Focus Group Study was designed in order to give input to modelling driving behavior in a simulation based evaluation framework. The main objective of the evaluation framework is to investigate how Look Ahead Cruise Control (LACC) influence the surrounding traffic with respect to driving behavior and fuel consumption. To be able to model driving behavior in the equipped vehicle, we need to discuss it with drivers who have experience in driving trucks equipped with LACC. More specifically, we will study when the drivers decide to deactivate or reactivate the cruise control. Several questions were formulated based on different traffic situations.

\section{Participants:}

15 drivers with experience in driving trucks equipped with LACC from Scanias transportlaboratorium took part in the Focus Group Study. They distribute goods between Scanisa's factories. These participants are not selected based on any qualifications, age or experience. The only selection criteria is having some experience in driving with LACC. The participants have not received any information or outline of the study beforehand. A usual route for these drivers is a round-trip between Södertälje and Vordingborg. The driving scheme is divided in two parts. There is a change-station in Ishøj in Denmark. A drive may be Södertälje- Ishøj or Ishøj-Vordingborg with a few hours of rest in Ishøj. The discussion leaders were in Ishøj when the drivers arrived in the station.

The ambition is to meet several drivers at the same time and arrange focus groups. To meet the drivers at the change-station in Ishøj make it possible. Another way to meet the drivers is to arrange a meeting with each driver. This would be more time consuming than having focus groups. Other advantage of discussion in focus groups is to have possibility to discuss the topics with other 
drivers. Three focus groups with 6,5 and 4 participants, were formed based on their arrival time to the station.

\section{Discussion plan:}

The FGS conducted took approximately 1.5 hours including refreshments. The meeting started with coffee, introducing the leaders and then start the discussion. Each focus group were conducted according to following procedure:

Welcome and information about the study, the meeting and time plan.

Presentation of the discussion leaders.

Introduction of the research project and the purpose of the focus group

How the discussion is planed.

Basic rule, the drivers are the "experts" and there are no right or wrong answers. We need to know how they experienced driving with the cruise control.

The meeting started with an introduction about the purpose of the meeting and indication that the aim of the focus group discussions is to find out how the participants have experienced driving with LACC. The FGS is a form of group interview which involves participants to discuss the decisions, observations and feelings when using LACC.

The group was informed that the session is recorded in order to avoid to miss any of their comments. Their thoughts and experiences are very valuable and helpful in these discussions and the meeting time is not long enough to write down everything. However, the recording is only used as a support for the discussion leaders and no one else will listen to the recording.

All opinions are important and everyone's engagement is appreciated. Some general questions will be asked and the drivers are welcome to discuss other topics that they think are valuable in this matter. 


\section{Questions}

Five general questions are used as guides for focus group discussions. For each question, a couple of situations are formulated to guide the discussions.

1. Which traffic situations do you decide not to set LACC ON?

a. Does the traffic density influence your decision?

b. Do you observe the traffic situations ahead in order to find out if you might be constrained by other vehicles?

c. Do you deactivate LACC if there is a risk for collision?

2) How do you react in a situation when you are constrained by the preceding vehicle?

a. What do you do if the preceding vehicle is slower and the LACC is activated?

b. What do you do if you are constrained by a platoon. When do you decide to join the platoon and when do you decide to overtake?

3) Assume that the road slope is not evident. Do you deactivate the LACC during an overtaking?

a. What do you do if it is an uphill/downhill?

b. What do you do if you are overtaking a platoon?

4) Have you noticed increased travel time when using LACC, compared with a CC?

a. If you have noticed an increase in travel time, is it acceptable?

b. Do you adjust the set-speed in order to compensate the longer travel time that LACC has caused?

c. Do you deactivate LACC more often than CC in order to compensate the longer travel time? 
d. Do you choose a higher set-speed for the Down Hill Speed Control (DHSC) in order to compensate the longer travel time?

5) Do you need to deactivate LACC when you are going to merge from an onramp?

a. Do you deactivate LACC in order to help a merging vehicle?

b. Do you change the lane in order to keep LACC ON when helping a merging vehicle?

c. Do you deactivate LACC when the preceding vehicle decelerates to exit the motorway?

\section{Outcome}

The outcome of the discussions has been summarized and listed in several statements below. This means that some of the participants may consider a statement. It does not mean that all of participants state same reaction or decision. Other participants may not observe any specific reaction in this case or do not agree with it.

- No participants state the increase in travel time caused by LACC make them to increase the set-speed in order to compensate the longer travel time.

- Many participants state that they do not deactivate LACC when the vehicle in constrained by a slower vehicle ahead. They estimate initially if an overtaking is possible. When it is not possible to overtake, they will press the brake pedal to adjust the speed. Many trucks have been equipped with Adaptive Cruise Control (ACC), which adapts the speed according the preceding vehicle if it is slower. Therefore, they do not have to for example press the brake pedal to decelerate and deactivate the LACC when ACC is activated. Some of the drivers state that they deactivate the ACC when lanechanging. 
- They indicate an enhanced functionality of LACC compared with the earlier version. For example, the speed do not decrease drastically in uphill, as it did in the beginning of the field observation test (FOT).

- They accelerate when overtaking takes too long time.

- The drivers will join a platoon if the platoon includes the trucks from the same company. Generally, it seems to be more difficult to join a platoon when driving with LACC, since it is not easy to adapt the speed after the platoon.

- In downhill, some of the drivers choose to deactivate LACC in order to keep the speed limitations.

- When there are several platoons ahead, the drivers deactivate LACC to be able to overtake the platoons.

- They have noticed a degeneration of LACC functionality when the weather condition is not normal, e.g. when it is raining or snowing in fog or damp.

- The system should take into account the vehicles mass and weight. They experience that the LACC cannot optimize the speed when the load is low.

- It is challenging to have LACC activated when platooning. It requires some speed adaptions using gas or brake pedal in order to keep the platoons speed. If the platoon leader has activated LACC, it will reduce the speed close to the top of hill. This makes the following vehicle to decelerate earlier and then have to accelerate to avoid drastic speed reduction. 


\section{Appendix II}

\section{Graphs \& Tables}

This appendix contains graphs and tables that show detailed measurements of the emissions, the fuel consumption and the travel time. 
Table 1. Comparison of the mean fuel consumption for trucks in scenarios with normal distribution, compared with scenarios with uniform distribution of the desired speed

\begin{tabular}{|c|c|c|c|c|c|}
\hline \multicolumn{6}{|c|}{ CC-equipped trucks } \\
\hline & & $0 \%$ LACC & $25 \%$ LACC & $50 \%$ LACC & $75 \%$ LACC \\
\hline truck flow $(\mathrm{v} / \mathrm{h})=\mathbf{2 0 0}$ & car flow (v/h) & $100 \% \mathrm{CC}$ & $75 \%$ CC & $50 \%$ CC & $25 \%$ CC \\
\hline uniform distribution & 800 & 3.281 & 3.273 & 3.273 & 3.267 \\
\hline Normal distribution & & 3.241 & 3.250 & 3.285 & 3.242 \\
\hline uniform distribution & 1600 & 3.488 & 3.515 & 3.532 & 3.601 \\
\hline \multirow[t]{2}{*}{ Normal distribution } & & 3.350 & 3.351 & 3.392 & 3.405 \\
\hline & & $0 \%$ LACC & $25 \%$ LACC & $50 \%$ LACC & $75 \%$ LACC \\
\hline truck flow $(v / h)=400$ & car flow (v/h) & $100 \%$ CC & $75 \%$ CC & $50 \%$ CC & $25 \%$ CC \\
\hline uniform distribution & 800 & 3.490 & 3.507 & 3.499 & 3.569 \\
\hline Normal distribution & & 3.445 & 3.479 & 3.488 & 3.531 \\
\hline uniform distribution & 1600 & 3.616 & 3.643 & 3.680 & 3.717 \\
\hline Normal distribution & & 3.606 & 3.630 & 3.654 & 3.702 \\
\hline
\end{tabular}

\section{LACC-equipped trucks}

$25 \%$ LACC $50 \%$ LACC $75 \%$ LACC $100 \%$ LACC

\begin{tabular}{|c|c|c|c|c|c|}
\hline truck flow $(\mathrm{v} / \mathrm{h})=200$ & car flow (v/h) & $75 \%$ CC & $50 \%$ CC & $25 \%$ CC & $0 \%$ CC \\
\hline uniform distribution & 800 & 3.281 & 3.273 & 3.273 & 3.267 \\
\hline Normal distribution & & 3.116 & 3.092 & 3.117 & 3.149 \\
\hline uniform distribution & 1600 & 3.488 & 3.515 & 3.532 & 3.601 \\
\hline Normal distribution & & 3.211 & 3.264 & 3.256 & 3.325 \\
\hline
\end{tabular}

\begin{tabular}{|c|c|c|c|c|c|}
\hline truck flow $(\mathrm{v} / \mathrm{h})=400$ & car flow (v/h) & $\begin{array}{c}25 \% \text { LACC } \\
75 \% \text { CC } \\
\end{array}$ & $\begin{array}{c}50 \% \text { LACC } \\
50 \% \text { CC } \\
\end{array}$ & $\begin{array}{c}75 \% \text { LACC } \\
25 \% \text { CC } \\
\end{array}$ & $\begin{array}{c}100 \% \text { LACC } \\
0 \% \text { CC } \\
\end{array}$ \\
\hline " uniform distribution & 800 & 3.490 & ב3.507 & 3.499 & 3.569 \\
\hline Normal distribution & & 3.331 & 3.342 & 3.404 & 3.387 \\
\hline uniform distribution & 1600 & 3.616 & 3.643 & 3.680 & 3.717 \\
\hline Normal distribution & & 3.496 & 3.524 & 3.554 & 3.581 \\
\hline
\end{tabular}


-200 trucks 800 cars /h

-200 trucks 1600 cars $/ \mathrm{h}$

-400 trucks 800 cars /h

-400 trucks 1600 cars $/ \mathrm{h}$
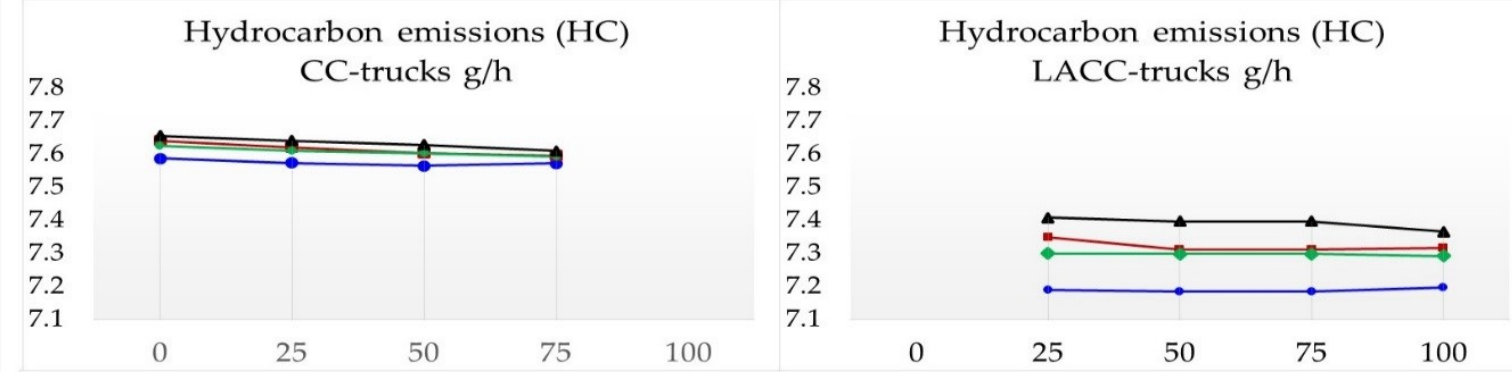

Carbon monoxide emissions (CO)

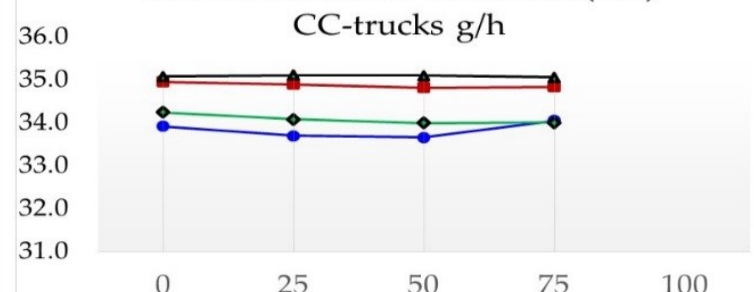

36.0

Carbon monoxide emissions (CO)

35.0

34.0

33.0

32.0

31.0

LACC-trucks g/h

Nitrogen Oxide emissions (NOx)

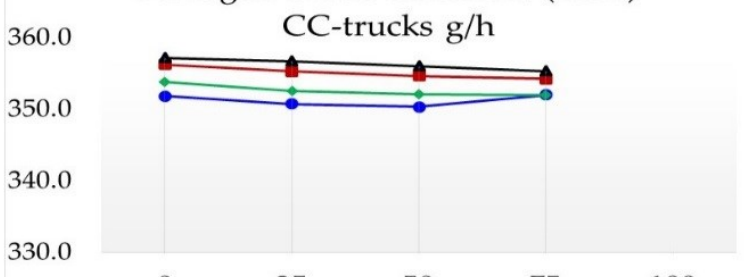

360.0

Nitrogen Oxide emissions (NOx)

350.0

340.0

330.0

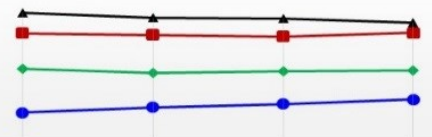

25

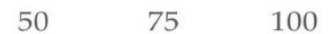

LACC-trucks $\mathrm{g} / \mathrm{h}$

PROCENT LACC-TRUCKS

Figure 1. Comparison of the traffic related emissions for LACC-trucks and CC-trucks 
Table 2. Comparison of the traffic related emissions

Difference (\%) in Hydrocarbon emissions (HC) for CC-truck compared to LACC-trucks

\begin{tabular}{cc|ccc}
\hline $\begin{array}{c}\text { truck flow } \\
\text { v/h }\end{array}$ & car flow v/h & $\begin{array}{c}\mathbf{2 5 \%} \text { LACC } \\
\mathbf{7 5 \%} \text { CC }\end{array}$ & $\begin{array}{c}\mathbf{5 0 \%} \text { LACC } \\
\mathbf{5 0} \mathbf{~ C C}\end{array}$ & $\begin{array}{c}\mathbf{7 5 \%} \text { LACC } \\
\mathbf{2 5} \% \text { CC }\end{array}$ \\
\hline 200 & 800 & 5.34 & 5.29 & 5.39 \\
200 & 1600 & 3.70 & 3.97 & 3.88 \\
400 & 800 & 3.15 & 3.13 & 2.88 \\
400 & 1600 & & &
\end{tabular}

\begin{tabular}{|c|c|c|c|c|}
\hline \multicolumn{5}{|c|}{ Difference (\%) in Carbon monoxide emissions (CO) for CC-trucks compared to LACC-trucks } \\
\hline truck flow & & $25 \%$ LACC & $50 \%$ LACC & $75 \%$ LACC \\
\hline $\mathbf{v} / \mathbf{h}$ & car flow $\mathrm{v} / \mathrm{h}$ & $75 \%$ CC & $50 \%$ CC & $25 \% \mathrm{CC}$ \\
\hline 200 & 800 & 6.56 & 6.02 & 6.97 \\
\hline 200 & 1600 & 4.25 & 4.11 & 4.29 \\
\hline 400 & 800 & 3.41 & 3.71 & 3.66 \\
\hline 400 & 1600 & 0.00 & 0.00 & 0.00 \\
\hline
\end{tabular}

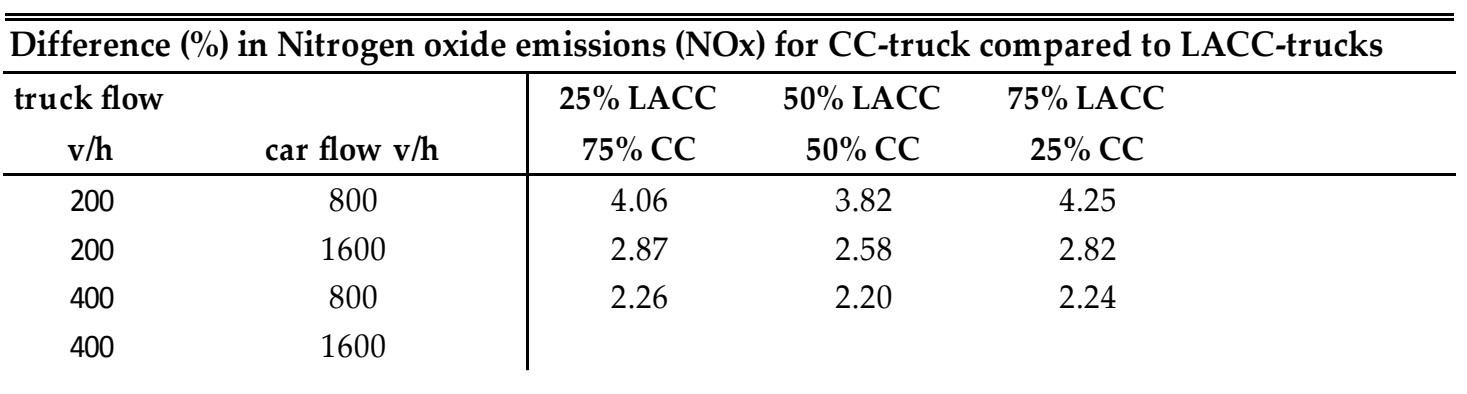



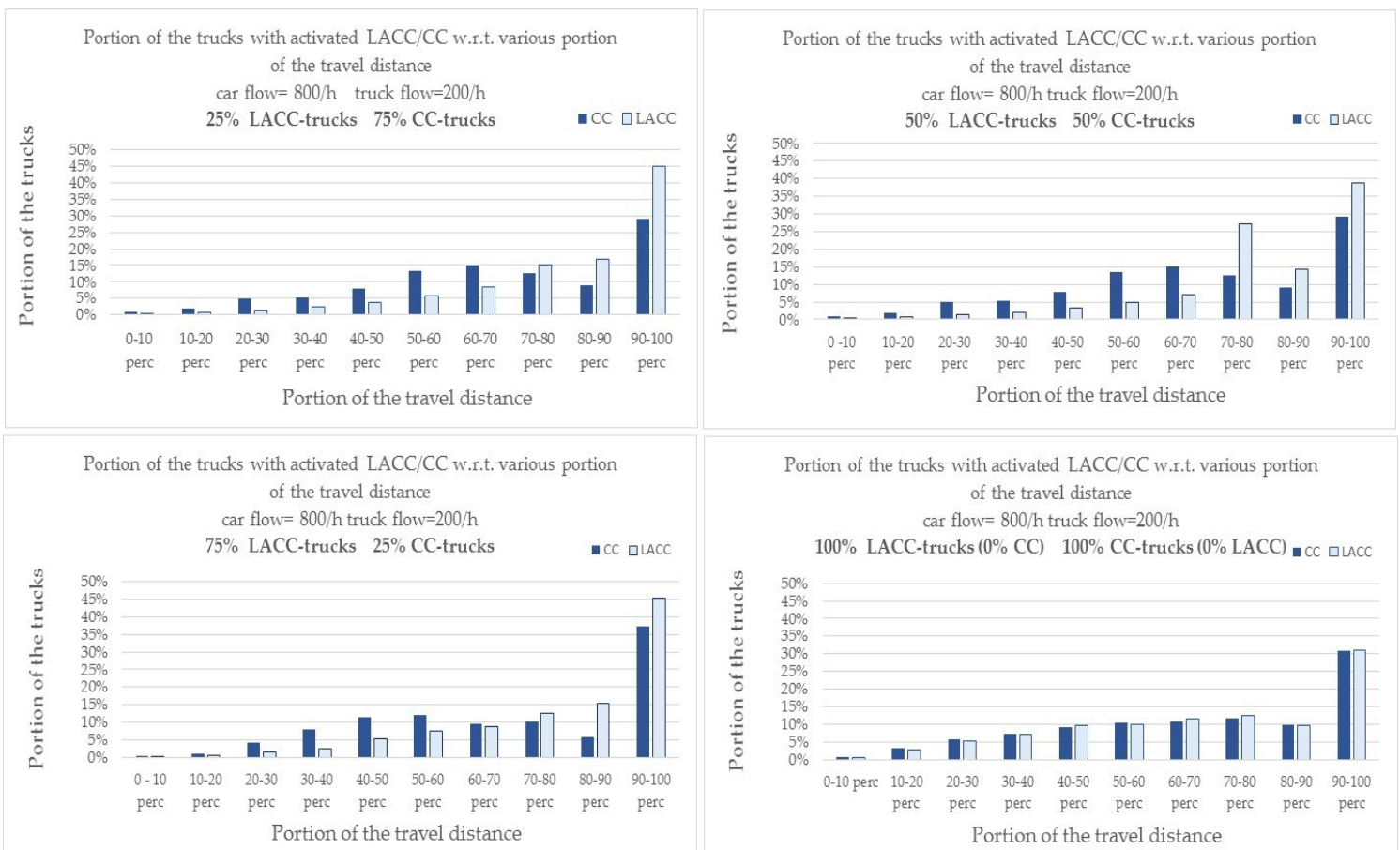

Figure 5. Portion of trucks that have LACC/CC activated, with respect to portion of the travel distance, car flow $=800 \mathrm{v} / \mathrm{h}$, truck flow $=200 \mathrm{v} / \mathrm{h}$

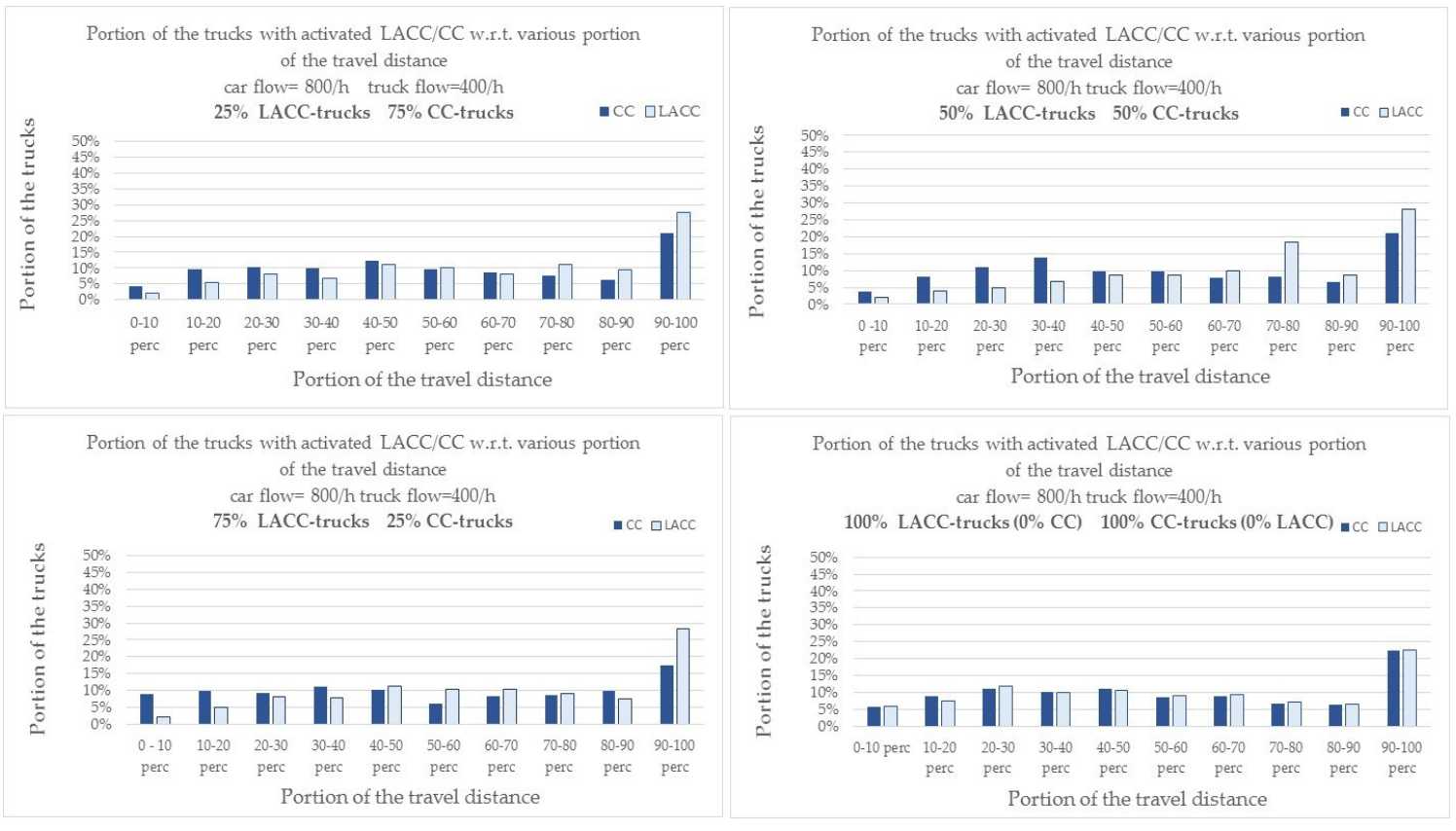

Figure 6. Portion of trucks that have LACC/CC activated, with respect to portion of the travel distance, car flow $=800 \mathrm{v} / \mathrm{h}$, truck flow $=400 \mathrm{v} / \mathrm{h}$ 


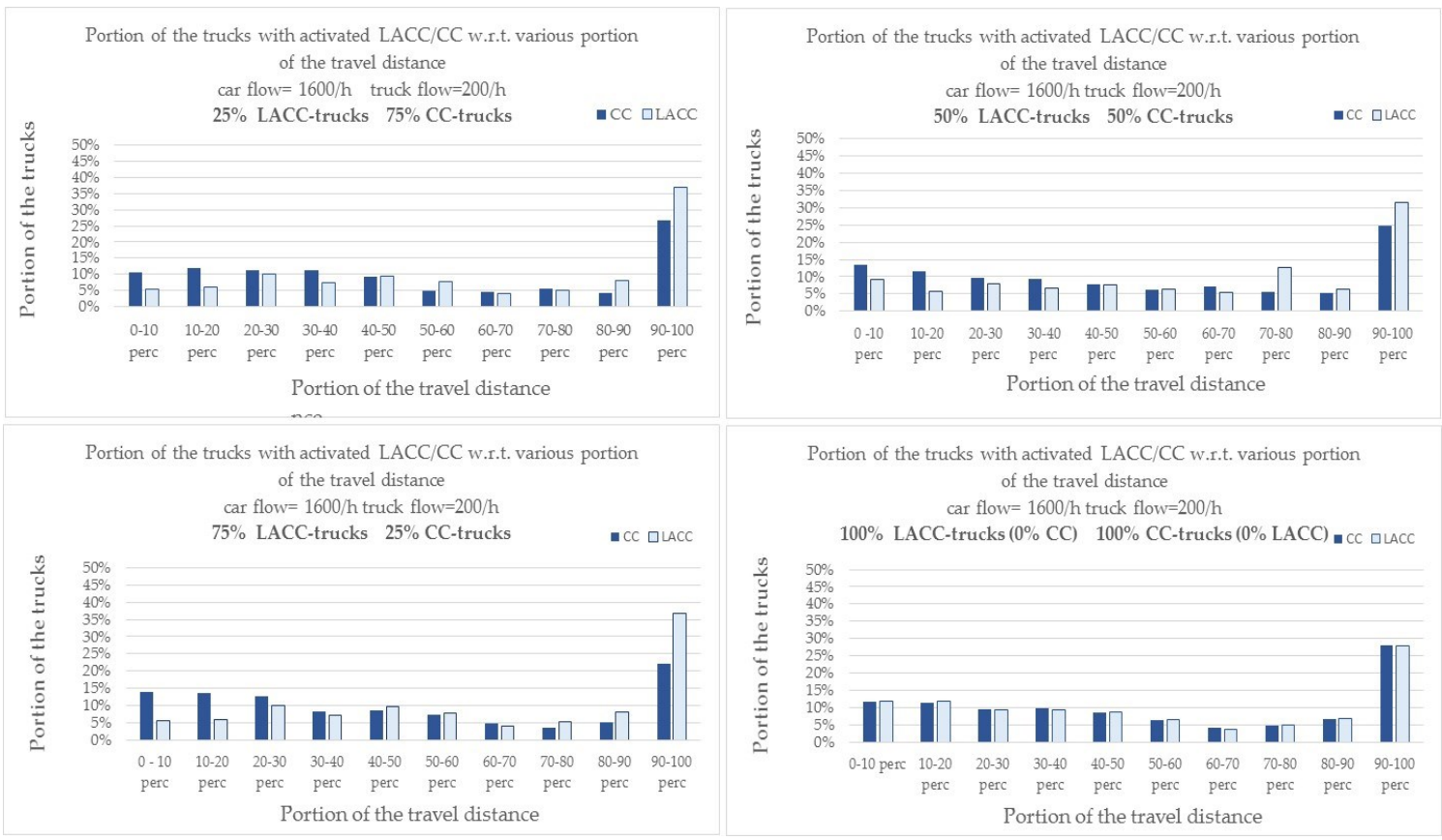

Figure 7. Portion of trucks that have LACC/CC activated, with respect to portion of the travel distance, car flow $=1600 \mathrm{v} / \mathrm{h}$, truck flow $=200 \mathrm{v} / \mathrm{h}$
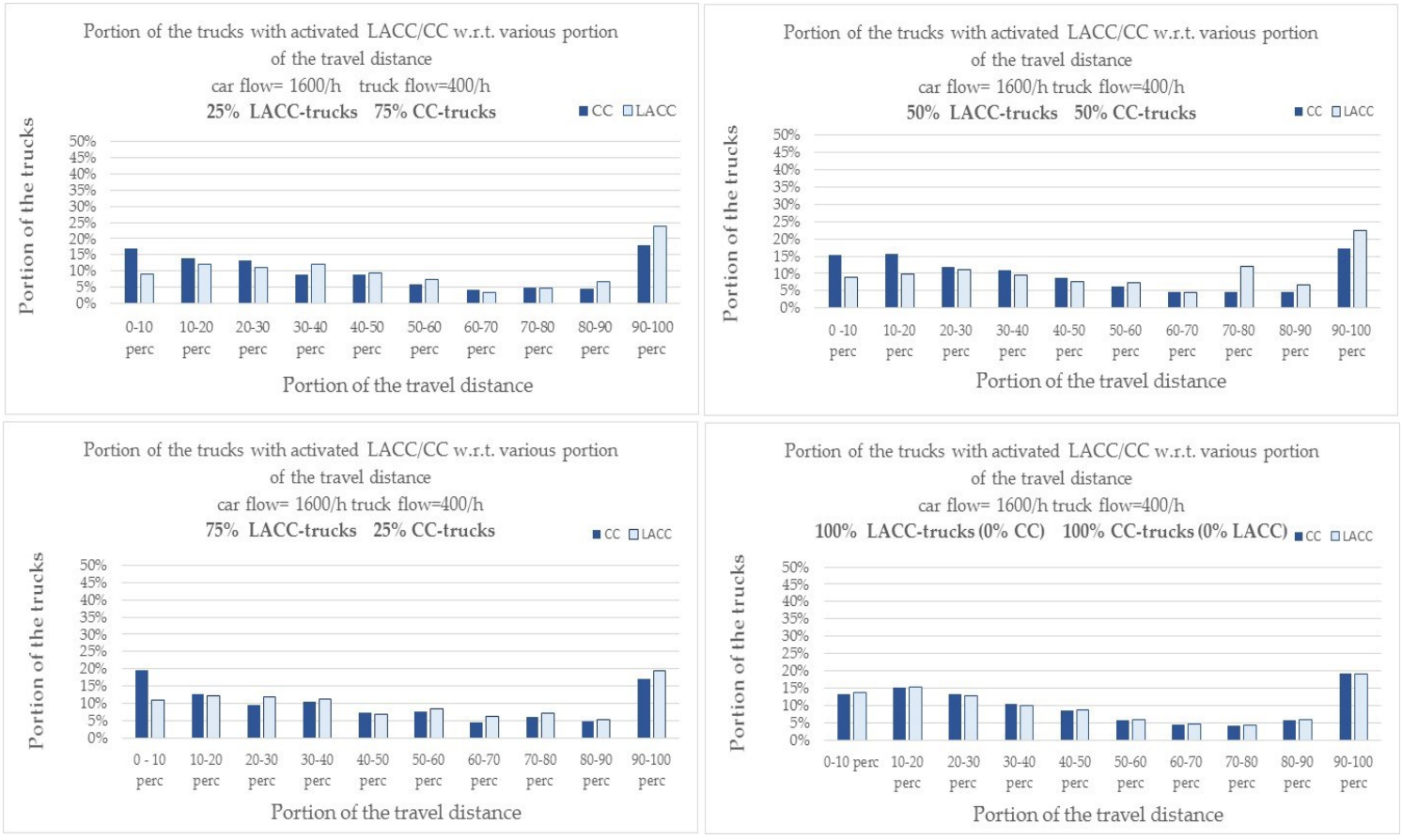

Figure 8. Portion of trucks that have LACC/CC activated, with respect to portion of the travel distance, car flow $=1600 \mathrm{v} / \mathrm{h}$, truck flow $=400 \mathrm{v} / \mathrm{h}$ 


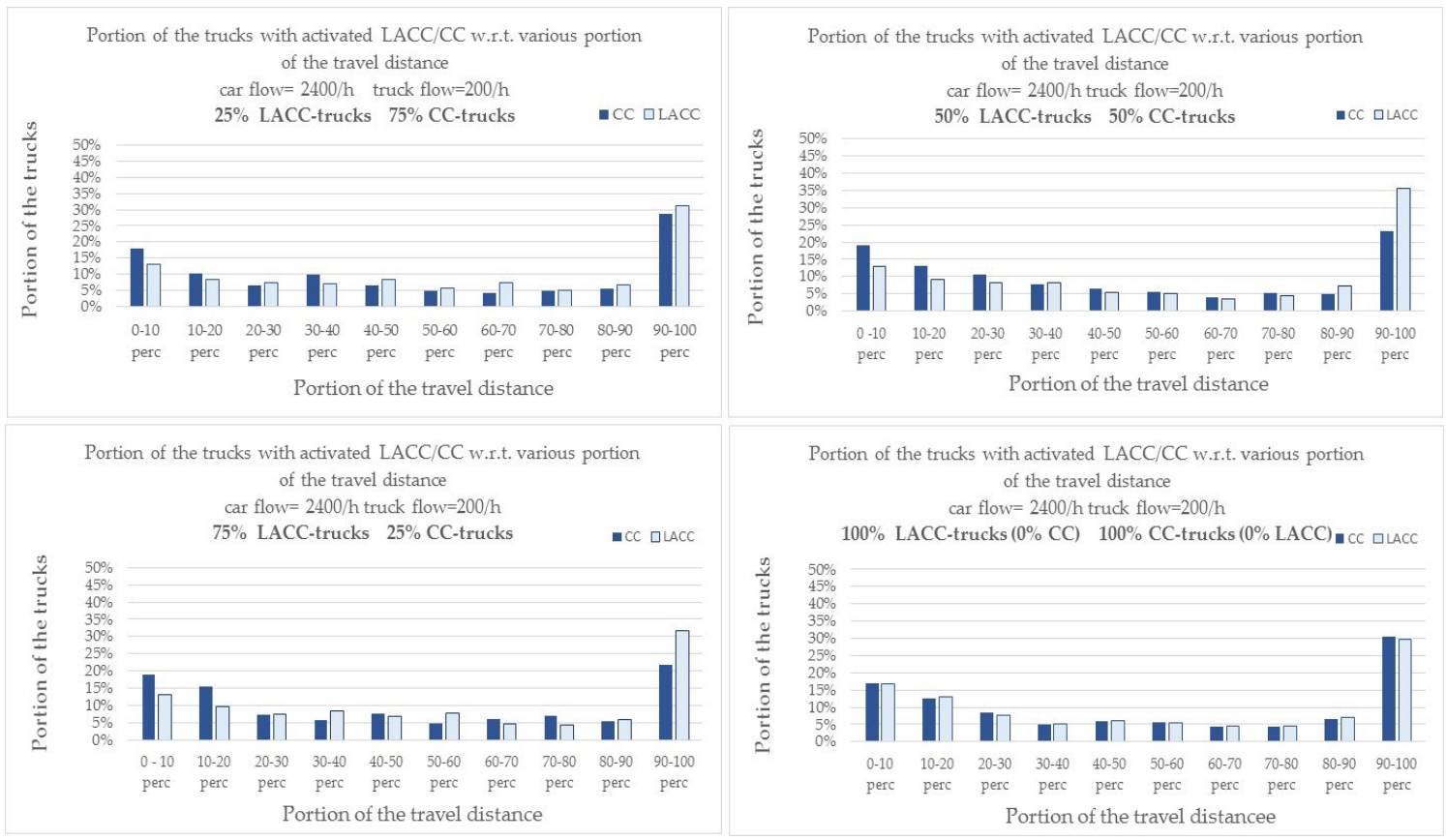

Figure 9. Portion of trucks that have LACC/CC activated, with respect to portion of the travel distance, car flow $=2400 \mathrm{v} / \mathrm{h}$, truck flow $=200 \mathrm{v} / \mathrm{h}$
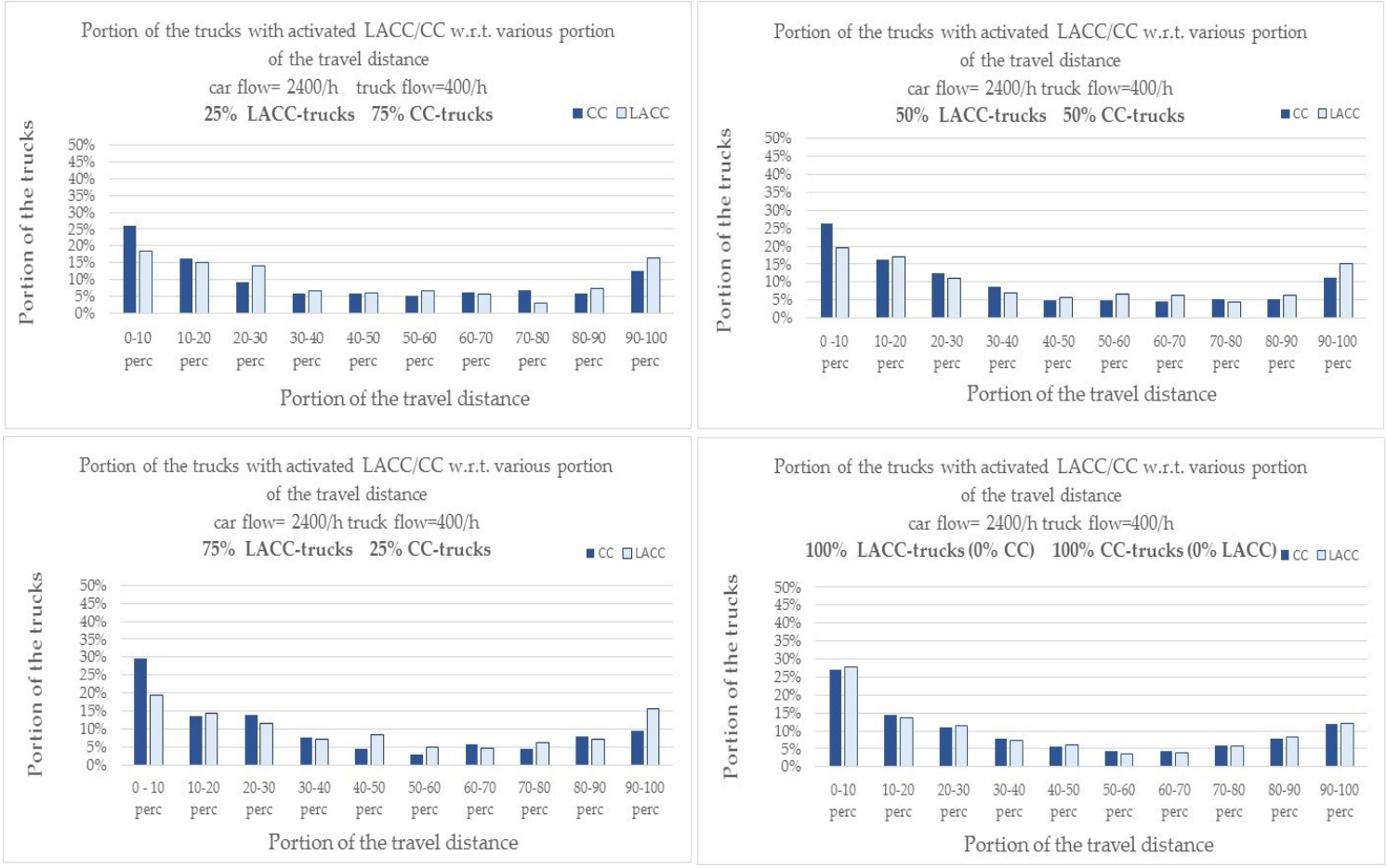

Figure 10. Portion of trucks that have LACC/CC activated, with respect to portion of the travel distance, car flow $=2400 \mathrm{v} / \mathrm{h}$, truck flow $=400 \mathrm{v} / \mathrm{h}$ 


\section{Bibliography}

AIDE (2005). "Review and Taxonomy of IVIS/ADAS applications." IST-1507674-IP.

Amundsen, F. and C. Hyden (1977). "Proceedings of first workshop on traffic conflicts,." Proc., 1st Workshop on Traffic Conflicts.

Barceló, J. (2010). Fundamentals of Traffic Simulation, volume 145 of International Series in Operations Research and Management Science, Springer.

Bertini, R. L. and A. El-Geneidy (2004). "ADVANCED TRAFFIC MANAGEMENT SYSTEM DATA."

Broqua, F. (1991). Cooperative driving: basic concepts and a first assessment of" intelligent cruise control" strategies. DRIVE Conference (1991: Brussels, Belgium). Advanced telematics in road transport. Vol. II.

Bång, K.-L., J. Olstam, J. Köhler, J. Wahlstedt, J. Andersson and A. Tapani (2014). "Handbok för kapacitetsanalys med hjälp av simulering." 
Casas, J., J. L. Ferrer, D. Garcia, J. Perarnau and A. Torday (2010). Traffic simulation with aimsun. Fundamentals of traffic simulation, Springer: 173-232.

Chaves, A. P., I. Steinmacher and V. Vieira (2011). "Social networks and collective intelligence applied to public transportation systems: A survey." VIII SBSC: 16-23.

Chorus, C. G., E. J. Molin and B. Van Wee (2006). "Use and effects of Advanced Traveller Information Services (ATIS): a review of the literature." Transport Reviews 26(2): 127-149.

D1.1.4, A. (2004). "Behavioural effects and driver-vehicle-environment modeling." IST-1-507674-IP.

Davis, L. (2004). "Effect of adaptive cruise control systems on traffic flow." Physical Review E 69(6): 066110.

Ericsson, E. (2001). "Independent driving pattern factors and their influence on fuel-use and exhaust emission factors." Transportation Research Part D: Transport and Environment 6(5): 325-345.

Fellendorf, M. and P. Vortisch (2010). Microscopic traffic flow simulator VISSIM. Fundamentals of Traffic Simulation, Springer: 63-93.

Gipps, P. G. (1981). "A behavioural car-following model for computer simulation." Transportation Research B 15(2): 105-111.

Gipps, P. G. (1981). "A behavioural car-following model for computer simulation." Transportation Research Part B: Methodological 15(2): 105-111.

Golias, J., C. Antoniou and G. Yannis (2002). "A methodology for the estimation of traffic and related impacts of advanced driver assistance systems." Its Journal 7(3-4): 261-277.

Hausberger, R. L. a. S. (2010). "User Guide for the Model PHEM."

Hegeman, G., A. Tapani and S. Hoogendoorn (2009). "Overtaking assistant assessment using traffic simulation." Transportation research part C: emerging technologies 17(6): 617-630. 
Hellström, E. (2010). "Look-ahead control of heavy vehicles."

Henclewood, D. and D. Ni (2009). "A dynamic interactive vehicle model for modelling traffic beyond the microscopic level." International journal of vehicle information and communication systems 2(1): 40-58.

Hengst, S. (2010). "VISSIM 5.30-00 Driver Model DLL Interface." PTV AG.

Henriksson P, H. M. (2012). Utvärdering av användarnas upplevelse av LACC.

Holtl, A. and S. Trommer (2013). "Driver Assistance Systems for Transport System Efficiency: Influencing Factors on User Acceptance." Iournal of Intelligent Transportation Systems 17(3): 245-254.

Hoogendoorn, S. P. and P. H. Bovy (2001). "State-of-the-art of vehicular traffic flow modelling." Proceedings of the Institution of Mechanical Engineers, Part I: Journal of Systems and Control Engineering 215(4): 283-303.

Kesting, A., M. Treiber, M. Schönhof, F. Kranke and D. Helbing (2007). Jamavoiding adaptive cruise control (ACC) and its impact on traffic dynamics. Traffic and Granular Flow'05, Springer: 633-643.

Klunder, G., M. Li and M. Minderhoud (2009). "Traffic Flow Impacts of Adaptive Cruise Control Deactivation and (Re) Activation with Cooperative Driver Behavior." Transportation Research Record: Journal of the Transportation Research Board 2129(1): 145-151.

Koupal, J., M. Cumberworth, H. Michaels, M. Beardsley and D. Brzezinski (2003). "Design and Implementation of MOVES: EPA's New Generation Mobile Source Emission Model." Ann Arbor 1001: 48105.

Liu, R. and J. Tate (2004). "Network effects of intelligent speed adaptation systems." Transportation 31(3): 297-325.

Maltby, D., S. Morello, K. Perrett and J. Hopkin (1998). "Checklist for preparing a validation plan: Updated version." CONVERGE project deliverable 2(1).

Michon, J. A. (1985). A critical view of driver behavior models: what do we know, what should we do? Springer. 
Minderhoud, M. M. (1999). Supported driving: impacts on motorway traffic flow.

Minderhoud, M. M. and P. H. Bovy (1999). "Impact of intelligent cruise control on motorway capacity." Transportation Research Record: Journal of the Transportation Research Board 1679(1): 1-9.

Olstam, J. J., J. Lundgren, M. Adlers and P. Matstoms (2008). "A framework for simulation of surrounding vehicles in driving simulators." ACM Transactions on Modeling and Computer Simulation (TOMACS) 18(3): 9.

Pauwelussen, J. and P. J. Feenstra (2010). "Driver behavior analysis during ACC activation and deactivation in a real traffic environment." Intelligent Transportation Systems, IEEE Transactions on 11(2): 329-338.

Pereira, J. L. and R. J. Rossetti (2012). An integrated architecture for autonomous vehicles simulation. Proceedings of the 27th Annual ACM Symposium on Applied Computing, ACM.

Piao, J. and M. McDonald (2008). "Advanced driver assistance systems from autonomous to cooperative approach." Transport Reviews 28(5): 659-684.

Pipes, L. A. (2004). "An operational analysis of traffic dynamics." Journal of applied physics 24(3): 274-281.

Punzo, V. (2011). "Integration of Driving and Traffic Simulation: Issues and First Solutions." IEEE transactions on intelligent transportation systems 12(2): 354363.

Rakha, H., M. Snare and F. Dion (2004). "Vehicle dynamics model for estimating maximum light-duty vehicle acceleration levels." Transportation Research Record: Journal of the Transportation Research Board 1883(1): 40-49.

Scania

"Scania

Active

Prediction."

http://www.scania.se/Images/Scania\%20Active\%20Prediction\%20\%20Presentation tcm85-287549.pdf.

Scholliers, J., S. Joshi, M. Gemou, F. Hendriks, M. Ljung Aust, J. Luoma, M. Netto, Engstro, x, J. m, S. L. Olsson, R. Kutzner, F. Tango, A. J. Amditis, J. M. 
Blosseville and E. Bekiaris (2011). "Development and Application of an Integrated Evaluation Framework for Preventive Safety Applications." Intelligent Transportation Systems, IEEE Transactions on 12(1): 211-220.

Scora, G. and M. Barth (2006). "Comprehensive modal emissions model (cmem), version 3.01." User guide. Centre for Environmental Research and Technology. University of California, Riverside.

Sivaraj, D., A. Kandaswamy, V. Rajasekar and P. Sankar Ganesh (2012). "VISION BASED AUTONOMOUS LATERAL AND LONGITUDINAL CONTROL SYSTEM." International Journal of Instrumentation \& Control Systems 2(4).

Statistical pocket book (2013). "EU transport in figures " http://ec.europa.eu/transport/facts-fundings/statistics/pocketbook2013 en.htm.

Tapani, A. (2011). Estimating traffic system wide impacts of driver assistance systems using traffic simulation. Human Modelling in Assisted Transportation, Springer: $173-180$.

Tapani, A. (2012). "Vehicle trajectory effects of adaptive cruise control." Journal of Intelligent Transportation Systems 16(1): 36-44.

Tapani, A., F. Pereyron and M. V. Turnell (2012). Integrated vehicle and traffic simulation for emissions and energy efficiency estimation.

Teetor, R. R. (1950). Speed control device for resisting operation of the accelerator, Google Patents.

Theil, H. (1966). "Applied economic forecasting." Studies in mathematical and managerial economics (4.

Treiber, M., A. Hennecke and D. Helbing (2000). "Congested traffic states in empirical observations and microscopic simulations." Physical Review E 62(2): 1805.

TSS-Transport, T.-T. S. S. (2011). "Aimsun 7 Microsimulator API Manual." TSSTransport Simulation Systems, Barcelona. 
Van Arem, B., A. De Vos and M. J. Vanderschuren (1997). "The microscopic traffic simulation model MIXIC 1.3."

Van Arem, B., C. J. van Driel and R. Visser (2006). "The impact of cooperative adaptive cruise control on traffic-flow characteristics." Intelligent Transportation Systems, IEEE Transactions on 7(4): 429-436.

van Driel, C. (2007). "Driver Support In Congestion." An assessment of user needs and impacts on.

van Noort, M., E. Jonkers, W. van den Haak and T. Ouboter (2011). Scaling up impact assessment results through statistics and simulation. Intelligent Transportation Systems (ITSC), 2011 14th International IEEE Conference on, IEEE.

Wiedemann, R. (1974). "SIMULATION DES STRASSENVERKEHRSFLUSSES."

Zhang, J. and P. A. Ioannou (2006). "Longitudinal control of heavy trucks in mixed traffic: environmental and fuel economy considerations." Intelligent Transportation Systems, IEEE Transactions on 7(1): 92-104. 